\title{
PERMEABILITY OF A SEGMENTED POLYETHER- POLYURETHANE TO DECAMETHYLTETRASILOXANE INTO BOVINE BLOOD
}

by

\author{
Greg Barber, B.Eng.
}

\author{
A thesis submitted to \\ the Faculty of Graduate Studies and Research \\ in partial fulfillment of \\ the requirements for the degree of \\ Master of Engineering (Mechanical) \\ Department of Mechanical and Aerospace Engineering \\ Ottawa-Carleton Institute \\ for Mechanical and Aerospace Engineering
}

\author{
Carleton University \\ Ottawa, Ontario \\ November 5, 1997 \\ (C) 1997, Greg Barber
}


National Library

of Canada

Acquisitions and Bibliographic Services

395 Wellington Street Ottawa ON KIA ON4 Canada
Bibliothèque nationale du Canada

Acquisitions et services bibliographiques

395, rue Wellington Ottawa ON K1A ONA
The author has granted a nonexclusive licence allowing the National Library of Canada to reproduce, loan, distribute or sell copies of this thesis in microform, paper or electronic formats.
L'auteur a accordé une licence non exclusive permettant à la Bibliothèque nationale du Canada de reproduire, prêter, distribuer ou vendre des copies de cette thèse sous la forme de microfiche/film, de reproduction sur papier ou sur format électronique.

L'auteur conserve la propriété du droit d'auteur qui protège cette thèse. $\mathrm{Ni}$ la thèse ni des extraits substantiels de celle-ci ne doivent être imprimés ou autrement reproduits sans son autorisation. 


\section{Carleton University

Thesis contains black and white and/or coloured graphs/tables/photographs which when microfilmed may lose their significance. The hardcopy of the thesis is available upon request from Carleton University Library. 


\title{
Confidential and Restricted Protected Business Information
}

\begin{abstract}
All information set forth herein is proprietary and is the intellectual property of the Cardiovascular Devices Division (CVD) of the University of Ottawa Heart Institute. Disclosure of any of this information in written or verbal form, without expressed written consent of the Director of CVD is strictly prohibited.
\end{abstract}




\begin{abstract}
Designing a reliable and effective ventricular assist device (VAD) presents many technical challenges. Problems include anatomical fit, sepsis, reliability, durability, low heat dissipation, high efficiency, minimal maintenance and monitoring. The HeartSaver $\mathrm{VAD}^{\mathrm{TM}}$ currently being developed by the Cardiovascular Devices Division of the University of Ottawa Heart Institute and World Heart Corporation is an innovative, new design which overcomes many of the technical challenges inherent to previous VAD designs.
\end{abstract}

The current design concept uses silicone oil as a hydraulic fluid to displace a flexible polyurethane diaphragm which pumps blood out of the device. This design concept overcomes some difficult problems with VAD design. However, it also raises questions relating to the fact that polyurethanes are typically highly permeable materials and the loss of hydraulic fluid may adversely effect the performance of the VAD.

This thesis documents an investigation into the permeability of silicone oil (decamethyltetrasiloxane) through a polymer diaphragm (Biospan - a segmented polyether-polyurethane) into whole bovine blood. The development of a test apparatus to determine the permeability rate of silicone oil through the diaphragm and a simple method for determining the diffusion coefficient of candidate biomaterials are presented. A method to extract silicone oil from whole blood for gas chromatography/mass spectrum (GC/MS) analysis is also discussed.

The results from the experiments show that the silicone oil/polyurethane/whole blood system has a permeability coefficient of $4.1 \times 10^{-11} \mathrm{~cm}^{2} / \mathrm{s}$, a diffusion coefficient of $3.1 \times 10^{-08} \mathrm{~cm}^{2} / \mathrm{s}$ and a solubility coefficient of .0013 . Using these values (which are specific only for the test model) the loss of silicone oil through a $60.5 \mathrm{~cm}^{2}$ diaphragm with a thickness of $.060 \mathrm{~cm}$ over one year would be $1.31 \mathrm{ml}$. The total volume of hydraulic fluid in the device is approximately $295 \mathrm{ml}$ and the loss of $1.31 \mathrm{ml}$ during a year is insignificant.

The thesis concluded that analysis of blood for silicone oil contamination can be performed using GC/MS. Silicone oil does permeate through segmented polyetherpolyurethanes at a slow rate. 


\section{ACKNOWLEDGMENTS}

This thesis would not have been possible without support from many sources. I would like to extend a warm thank you to the following people who were instrumental in the completion of my thesis, Don Russell, Tofy Mussivand, Robert Burk, Mike King, Roz Labow, Sherry Perkins, Albert Hum, Chuong Ho and Moheb Ibrahim.

I would also like to thank the 'Bank of Barber' (Rob Barber) and my mom, Merle Barber for their support all of the way through my University Education. I couldn't have done it without you. Finally, I would like to thank my brother Scott for several endless evenings of mischief which helped me keep my sanity and relax. 


\section{TABLE OF CONTENTS}

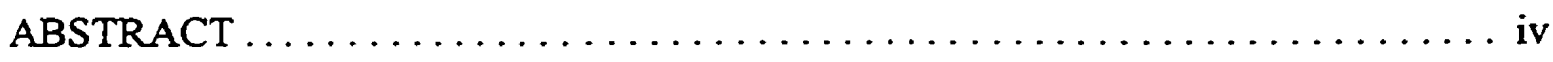

ACKNOWLEDGMENTS $\ldots \ldots \ldots \ldots \ldots \ldots \ldots \ldots \ldots \ldots \ldots \ldots \ldots$

TABLE OF CONTENTS $\ldots \ldots \ldots \ldots \ldots \ldots \ldots \ldots \ldots \ldots \ldots \ldots \ldots \ldots \ldots \ldots \ldots \ldots$

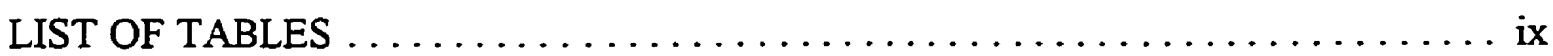

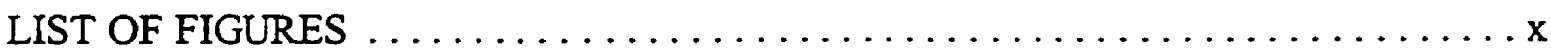

LIST OF SYMBOLS AND ACRONYMS $\ldots \ldots \ldots \ldots \ldots \ldots \ldots \ldots \ldots \ldots \ldots \ldots$ xii

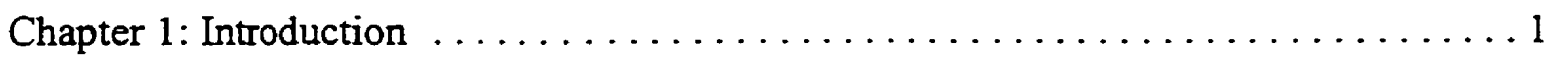

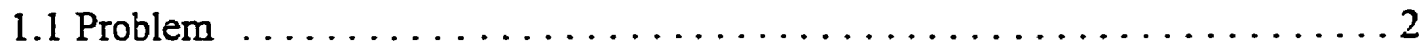

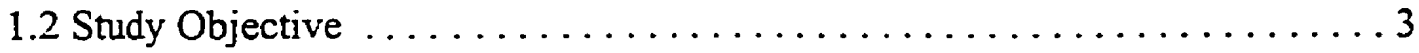

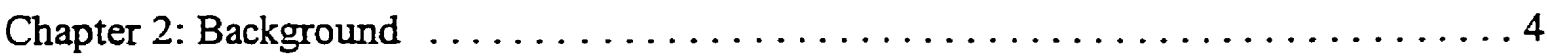

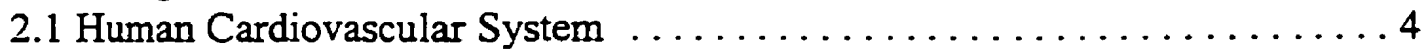

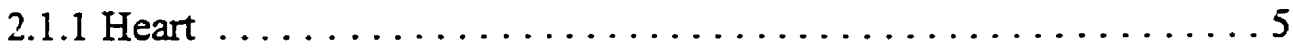

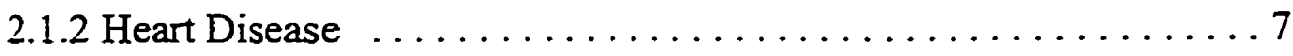

2.1.3 Blood Vessels and Blood Pressure . . . . . . . . . . . . . 8

2.2 Artificial Hearts \& Mechanical Assist Devices ................. 9

2.2.1 Technical Challenges . . . . . . . . . . . . . . . . . 10

2.2.2 Types \& Manufacturers . . . . . . . . . . . . . . . . . 11

2.2.3 HeartSaver Ventricular Assist Device (HSVAD) . . . . . . . 13

2.3 Blood ........................................ 15

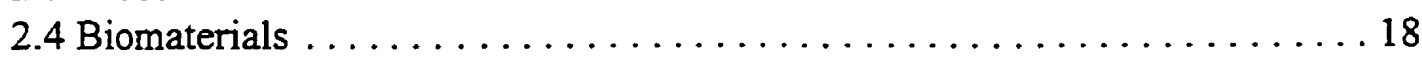

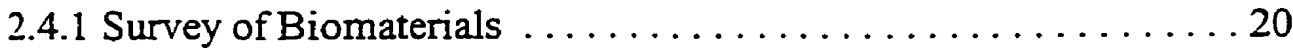

2.4.2 Segmented Polyether-Polyurethane (Biospan) ......... 20

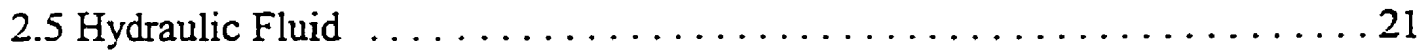

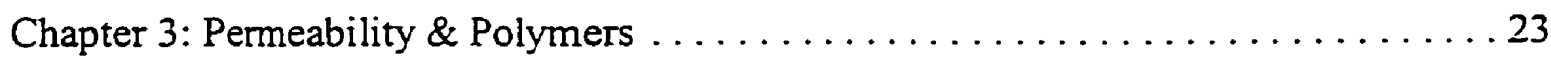

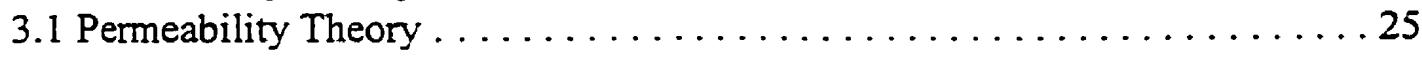

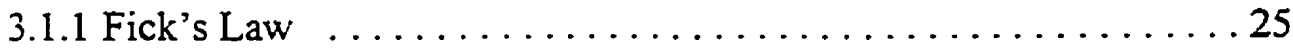

3.1.1.1 Diffusion into a Thin Film ................. 29

3.1.2 External Factors Affecting Permeability ............... 31

3.1.3 Polymer Characteristics Affecting Permeation ............ 33

3.1.4 Permeating Species Characteristics Affecting Permeation ...... 36 
3.2 Literature Review - Permeability/Diffusion/Sorption ........... 37

3.2.1 Silicone Oil Permeation ....................... 38

3.2.2 Water Vapour/Gas Permeation ................... 43

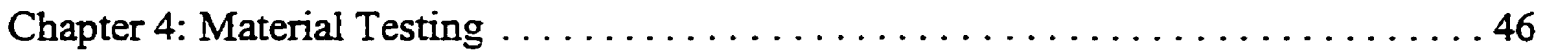

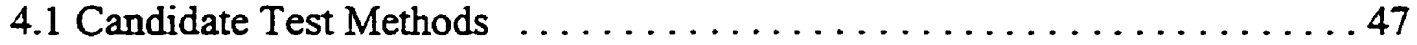

4.2 Selection of Parameters ........................... 50

Chapter 4.2.1 Selection of Parameters - Test Evaluation ......... 50

Chapter 4.2.2 Selection of Parameters - Absorption Test .......... 51

Chapter 4.2.3 Selection of Parameters - Permeability Test ........ 52

4.3 Expected Test Results .......................... 56

Chapter 4.3.1 Expected Test Results - Test Evaluation ............ 57

Chapter 4.3.2 Expected Test Results - Absorption Test .......... 58

Chapter 4.3.3 Expected Test Results - Permeability Test ......... 59

Chapter 5: Experimental Procedure . ......................... 61

5.1 Experimental Procedure - Test Evaluation $\ldots \ldots \ldots \ldots \ldots \ldots \ldots \ldots \ldots$

5.1.1 Sample Preparation ........................... 61

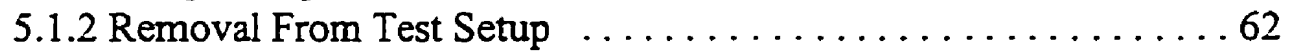

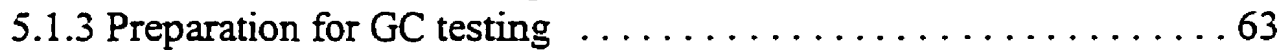

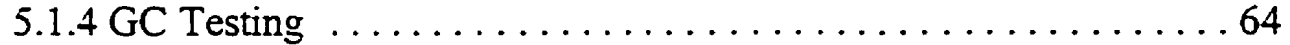

5.2 Experimental Procedure - Absorption Test .................6. 65

5.2.1 Absorption Procedure 1: GC Test End-Cap ............6. 65

5.2.1.1 Sample Preparation .....................65 65

5.2 .1 .2 Test Setup .........................66

5.2.1.3 Removal From Test Setup ...............67 67

5.2.1.4 Preparation For GC Testing ...............668

5.2.1.5 GC Testing ....................... 69

5.2.2 Absorption Procedure 2: GC Test Sheet ............ 70

5.2.2.1 Sample Preparation .................... 70

5.2.2.2 Test Setup ...................... 70

5.2.2.3 Removal From Test Setup ................ 71

5.2.2.4 Preparation For GC Testing . . . . . . . . . . . . 71

5.2.2.5 GC Testing ........................ 72

5.2.3 Absorption Procedure 3: Gravimetric Tests . . . . . . . . 73

5.2.3.1 Sample Preparation ..................... 73

5.2.3.2 Test Setup ..................... 73

5.2.3.3 Removal \& Return To Test Setup .............. 73

5.3 Experimental Procedure - Permeability Test ................ 74

5.3.1 Sample Preparation . . . . . . . . . . . . . . . . . . 74

5.3 .2 Test Setup .............................. 75

5.3.3 Removal from Test Setup .................... 76 
5.3.4 Preparation for GC Testing $\ldots \ldots \ldots \ldots \ldots \ldots \ldots \ldots \ldots \ldots \ldots \ldots$

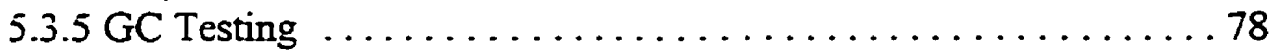

Chapter 6: Results and Calculations ........................ 80

6.1 Observations and Results - Test Evaluation $\ldots \ldots \ldots \ldots \ldots \ldots \ldots$

6.1.1 Data Acquisition and Quantitative Results - Test Evaluation . . . 80

6.1.2 Observations and Qualitative Results - Test Evaluation ....... 83

6.2 Results - Absorption Test . ...................... 83

6.2.1 Data Acquisition and Quantitative Results - Absorption Test . . . 84

6.2.2 Observations and Qualitative Results - Absorption Test ......99 91

6.3 Observations and Results - Permeability Test ............... 93

6.3.1 Data Acquisition and Quantitative Results - Permeability Test ...93

6.3.2 Observations and Qualitative Results - Permeability Test ...... 98

Chapter 7: Discussion of Results ............................ 100

7.1 Discussion of Results - Test Evaluation $\ldots \ldots \ldots \ldots \ldots \ldots \ldots \ldots \ldots$

7.2 Discussion of Results - Absorption Test .................. 102

7.3 Discussion of Results - Permeability Test ................... 104

Chapter 8: Conclusions \& Recommendations $\ldots \ldots \ldots \ldots \ldots \ldots \ldots \ldots \ldots \ldots$

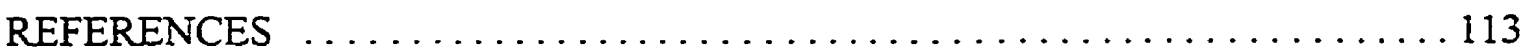

APPENDIX A:

Experimental Apparatus ............................ 119

APPENDIX B:

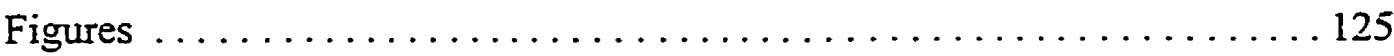

APPENDIX C:

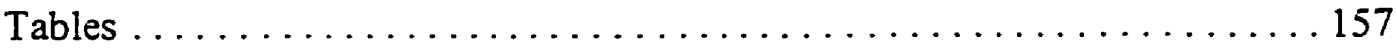

APPENDLX D:

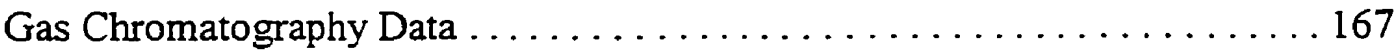




\section{LIST OF TABLES}

Page

Table 1 Comparison of Human \& Bovine Blood 158

Table 2 Bio-compatible Elastomers 158

Table 3 Biospan - Material Characteristics 158

Table $4 \quad$ Test Evaluation Results 159

Table 5 Absorption Test Data - GC/MS End-Cap Test 160

Table 6 Absorption Test Data - GC/MS Sheet Test 161

Table 7 Absorption Test Data - Gravimetric Test I 162

Table 8 Absorption Test Data - Gravimetric Test II 163

Table 9 Absorption Test Data - Gravimetric Test Coefficients 164

Table $10 \quad$ Permeability Test - Data 165

Table $11 \quad$ Permeability Test - Permeability Coefficients 166

Table 12 Predicted Volume of Silicone Oil Loss Through Blood 166

Chamber of the HSVAD

Table 13 GC Method - Permeability Test 168

Table 14 ACU Method - Permeability Test 168

Table 15 Gas Chromatography Sample Mass List - Standard $\quad 171$

Table 16 Gas Chromatography Sample Mass List - Sample PV12 174 


\section{LIST OF FIGURES}

Figure 1

Figure 2

Figure 3

Figure 4

Figure 5

Figure 6

Figure 7

Figure 8

Figure 9

Figure 10

Figure 11

Figure 12

Figure 13

Figure 14

Figure 15

Figure 16

Figure 17

Figure 18

Figure 19

Figure 20

Figure 21

Figure 22

Figure 23

Figure 24

Figure 25

Figure 26

Figure 27

Figure 28

Figure 29

Figure 30

Figure 31

Figure 32

Figure 33

Figure 34
Circulatory System Diagram

126

Heart Cross Section $\quad 127$

Heart Valves

127

Vessel Diagram

128

EVAD Location in Body

129

$\begin{array}{ll}\text { EVAD Connection Diagram } & 130 \\ \text { Blood Chamber and VDC Diagram Placement } & 131\end{array}$

Blood Chamber Diaphragm 131

Unassembled Device $\quad 132$

VDC Diaphragm 132

Assembly Diagram of Test Apparatus 133

Biospan Molecule 134

Decamethyltetrasiloxane (Silicone Oil) Molecule $\quad 134$

Permeation Through Film Curve Approaching 135

Steady State

135

136

136

137

137

138

138

139

139

140

140

141

141

142

142

143

144

145

146

147 
Figure 35 Graph Absorption Test - Gravimetric Data 148 (Solubility Graph) II

Figure 36 Graph Absorption Test - Gravimetric Data 149 (Fractional Absorption Graph) I

Figure 37 Graph Absorption Test - Gravimetric Data 150

Figure 38 Graph Absorption Test - Combined Absorption 151 Test Results

Figure 39 Graph Permeability Test Data (18 days) 152

Figure $40 \quad$ Graph Permeability Test Data (6 days) 153

Figure 41 Comparison of Permeability Test Blood Before 154 Testing and After $6 \mathrm{D}$

Figure 42 Mixed Solvents DCM \& Hexane with Blood (Inverted) 154

Figure 43 Centrifuged Hexane and Blood Sample 155

Figure 44 Mixed Solvents DCM and Hexane with Blood 155

Figure 45 Centrifuged DCM and Blood Sample 156

Figure $46 \quad$ Gas Chromatography Sample Graph - Standard 169

Figure 47 Gas Chromatography Mass Spectrum Graph - Standard 170

Figure $48 \quad$ Gas Chromatography Sample Graph - Sample PV12 172

Figure 49 Gas Chromatography Mass Spectrum Graph - 173

Sample PV12 


\section{LIST OF SYMBOLS AND ACRONYMS}

\begin{tabular}{|c|c|}
\hline A & area \\
\hline $\mathrm{ACE}$ & Angiotensin Converting Enzyme \\
\hline ACU & acquisition method \\
\hline Area $_{\mathrm{GC}}$ & area of silicone oil peak on $G C$ graph \\
\hline$C_{1}$ & concentration at the permeant face \\
\hline $\mathrm{C}_{2}$ & concentration at the receiving face \\
\hline $\mathrm{C}_{\mathrm{d}}$ & concentration in the donor chamber \\
\hline$C_{\text {dilext }}$ & concentration of silicone oil in the diluted volume of extraction solvent \\
\hline $\mathrm{C}_{\mathrm{ext}}$ & concentration of sample \\
\hline $\mathrm{C}_{\mathrm{r}}$ & concentration in the receiving chamber \\
\hline CPDA & Citrate Phosphate Dextrose Adenine \\
\hline Coef $_{\text {snd }}$ & coefficient standard \\
\hline $\mathrm{CVD} / \mathrm{OHI}$ & $\begin{array}{l}\text { Cardiovascular Devices Division of the University of Ottawa Heart } \\
\text { Institute }\end{array}$ \\
\hline D & diffusion coefficient \\
\hline DCM & dichloromethane solvent \\
\hline DMAC & dimethylacetamide \\
\hline DMF . & dimethylformamide \\
\hline EVAD & Electrohydraulic Ventricular Assist Device \\
\hline $\mathrm{F}$ & flux \\
\hline FTIR & Fourier Transform Infrared Spectroscopy \\
\hline GC & gas chromatography \\
\hline HPLC & $\begin{array}{l}\text { High Performance Liquid Chromatography or High Pressure Liquid } \\
\text { Chromatography }\end{array}$ \\
\hline HSVAD & HeartSaver Ventricular Assist Device \\
\hline LVAD & Left Ventricular Assist Device \\
\hline $\mathrm{M}_{\mathrm{t}}$ & mass uptake at a given time \\
\hline$M_{t=0}$ & initial mass of the sample \\
\hline $\mathrm{M}_{\mathrm{f}}$ & maximum mass uptake \\
\hline MS & mass spectrum \\
\hline $\mathrm{P}$ & permeability coefficient \\
\hline PBS & phosphate buffered saline \\
\hline ppb & parts per billion \\
\hline ppm & parts per million \\
\hline PTFE & Teflon \\
\hline & \\
\hline
\end{tabular}




$\begin{array}{ll}\text { RBC } & \text { Red Blood Cells } \\ S & \text { solubility coefficient } \\ S_{t} & \text { solubility at a time }(t) \\ \text { TAH } & \text { Total Artificial Heart } \\ \text { TET } & \text { transcutaneous energy transfer } \\ \text { VAD } & \text { ventricular assist device } \\ \text { VDC } & \text { volume displacement chamber } \\ V_{\text {diled }} & \text { diluted volume of sample } \\ V_{\text {dilm }} & \text { volume of pure solvent to dilute sample } \\ V_{\text {ext }} & \text { total volume of extraction solvent } \\ V_{\text {inj }} & \text { volume of sample injected into GC } \\ V_{S I} & \text { volume of silicone oil in the total volume of extraction solvent } \\ \text { WBC } & \text { White Blood Cells } \\ X, Y & \text { variables } \\ c & \\ l & \text { concentration } \\ r_{\text {total }} & \text { thickness } \\ r_{r} & \text { total diffusion resistance } \\ r_{d} & \text { resistance of the receiving chamber } \\ r_{p} & \text { resistance of the donor chamber } \\ \pi & \text { resistance of the polymer membrane } \\ \text { silicone oil } & \text { constant }=3.14 \\ \text { slope } & \text { decamethyltetrasiloxane } \\ t & \text { linear approximation of the slope of the permeability test graph } \\ \tau & \text { time } \\ x, y, z & \text { lag time } \\ & \text { displacement direction }\end{array}$




\section{Chapter 1: Introduction}

The leading cause of death in Canada, and in most of the developed world is cardiovascular disease. One effective treatment for end-stage heart disease is transplantation. Transplantation is hardly the perfect solution since donor organs are limited and complications arise from immune suppressant drugs which deteriorate the immune system and leave transplant patients with a reduced quality of life. (HSVAD Product Literature, 1997)

In Canada alone, 45,000 people die each year from heart disease and only about 3,000 people world-wide receive heart transplants each year. Through the use of ventricular assist devices (VADs) some of the people who died could have had their lives extended. The Cardiovascular Devices Division of the University of Ottawa Heart Institute (CVD/OHI), in cooperation with World Heart Corporation, is developing a VAD that can be used for long-term circulatory support. Availability of VADs is not restricted as it is with donor hearts. VADs also avoid problems associated with immune suppressant drugs required for transplant patients. The lack of immune suppressant drugs makes the VAD more economical, results in a better quality of life and a better chance for recovery for VAD patients. (HSVAD Product Literature, 1997) 
Ongoing testing has shown that ventricular assist devices can be used effectively to treat end-stage heart disease. (Hill et. al., 1993) Unfortunately, technical challenges have limited the use of VADs to bridge to transplant cases. The HeartSaver VAD ${ }^{\mathrm{TM}}$ (HSVAD) being developed by World Heart Corporation is intended for long-term circulatory support with the alternative as a temporary bridge to transplant. It is believed that this device has overcome many of the design problems associated with previous artificial heart and VAD technology. (HSVAD Product Literature, 1997)

\subsection{Problem}

A ventricular assist device must be capable of assuming the pumping abilities of the human heart. The human heart is an astounding pump capable of pumping $3,000,000$ liters of blood and beating 40,000,000 times per year. A mechanical device must match these performance characteristics with exceptional reliability. (HSVAD Product Literature, 1997)

In addition to matching these performance goals, the device must meet other requirements including anatomical fit, size and position constraints, surgical simplicity, biocompatibility, minimal costs, patient mobility, minimized maintenance, and energy and information transfer in and out of the body without perforating the skin. (HSVAD Product Literature, 1997)

In the process of designing a ventricular assist device to met all of these goals, the design team at the $\mathrm{CVD} / \mathrm{OHI}$ chose a design which used silicone oil as a hydraulic fluid to drive a diaphragm which pumps blood through the VAD. The selection of an effective blood pump diaphragm is a difficult task since the material must meet many requirements. 
Some of these requirements include excellent bio-compatibility, resistance to thrombosis (clotting of blood), fatigue, degradation and minimal permeability to fluids and gases. (McMillin, 1983)

The permeability of the blood pump diaphragm is an important issue since the loss of a large volume of silicone oil through the diaphragm would result in degraded performance and efficiency of the VAD. If a significant volume of silicone oil is lost a refilling port may be required to replace the lost fluid after it has been implanted and operated for given period of time.

\subsection{Study Objective}

The objective of this thesis was to determine if the silicone oil (decamethyltetrasiloxane) could permeate through the blood chamber diaphragm (Biospan - segmented polyetherpolyurethane) into the blood and if it does permeate through the diaphragm, then at what rate of permeation. A test method and apparatus was developed to predict the volume of silicone oil permeating through the diaphragm.

The test method was designed to analyze individual factors which influenced the permeability rate of fluid out of the VAD. The factors affecting permeability that were tested in this thesis included temperature, diaphragm thickness, donor fluid, receiving fluid and boundary conditions. The effects not taken into consideration were fluctuating pressure, fluctuating stress and flexing of the diaphragm. The apparatus is also capable of testing new candidate biomaterials to determine their suitability for use in the VAD with respect to permeability. 


\section{Chapter 2: Background}

This chapter provides background information on the cardiovascular system, artificial hearts and ventricular assist devices, blood, polymer materials and hydraulic fluids. This information is provided to help the reader understand how the permeability problem in this thesis is related to the overall implanted VAD system. Each of the topics discussed in this chapter influences the characteristics or requirements of the implanted device.

\subsection{Human Cardiovascular System}

The human cardiovascular system consists of the heart and blood vessels. This system is the infrastructure that moves blood throughout the body in an effort to supply cells with nutrients and oxygen and to remove cell wastes. The heart is a pump that creates a pressure rise in the aorta driving blood through the vessels of the body. In fact, the heart is two pumps. The right side of the heart pumps to the pulmonary circuit and the left side pumps to the systemic circuit (see Figure 1). (Marieb, 1995) 


\subsubsection{Heart}

The heart is located in the chest cavity, above the diaphragm. It is surrounded by the lungs and suspended on the left side of the chest between the second rib and the fifth intercostal space. The heart is approximately the size of a fist and weighs between 250 and 350 grams (Marieb, 1995).

The heart consists of 4 chambers: right atrium, right ventricle, left atrium and left ventricle. The left side of the heart is the strongest since it must pump blood through the entire body, with the exception of the lungs. The lungs receive blood that is pumped by the right side of the heart. The resistance to blood flow through the systemic circuit is 5 times greater than the resistance in the pulmonary side. As a result, the left side of the heart works much harder than the right side (Marieb, 1995).

The heart muscle is a special type of muscle due to its unique operating conditions. The heart muscles must work continuaily for the entire life span of a person. The average heart rate for an adult is 75 beats per minute. Therefore, in one year, a human heart will beat approximately 40 million times without taking a break (Marieb, 1995).

The atria are receiving chambers located at the top of the heart (see Figure 2) which collect the blood that is returning to the heart. They are much smaller than the ventricles and do not provide much pumping power. The right atrium receives the blood returning from the systemic circuit through the coronary sinus, superior vena cava and the inferior vena cava. The superior vena cava returns blood from vessels above the diaphragm while the inferior vena cava returns blood from vessel below the diaphragm. The coronary 
sinus returns blood that is pumping through the heart muscles. The left atrium receives blood that is returning through the pulmonary veins from the lungs. (Marieb, 1995)

The ventricles are the work horses of the heart (see Figure 2). They perform most of the pumping action required to move blood through the body. The left ventricle is larger than the right ventricle due to higher resistance in the systemic circuit. Blood is fed into the ventricles through valves located between each ventricle and its corresponding atrium. Blood that is pumped from the left ventricle leaves through the aorta, which is the largest artery in the body, and travels to the systemic circuit. Blood leaving the right ventricle leaves through the pulmonary trunk which breaks off into branches that lead to the lungs (pulmonary circuit). (Marieb, 1995)

Each of the four chambers in the heart has a valve at its exit to prevent the flow of blood back into the chamber after it has completed pumping. All of these valves operate passively depending on a pressure gradient. When the pressure is higher inside the chamber than outside of the chamber, the valve is opened. When the pressure starts to drop off, and the pressure outside of the chamber is higher than the pressure inside of the chamber, the valve automatically inflates like a parachute, sealing off the chamber and preventing any regurgitation of blood. The valve leaving the right atrium is called the tricuspid or right atrioventricular valve. The valve leaving the left atrium is called the bicuspid, mitral or left atrioventricular valve. The valve leaving the right ventricle is called the pulmonary semilunar valve and the valve leaving the left ventricle is called the aortic semilunar valve (see Figure 3). The atrioventricular valves, bicuspid and tricuspid, actually have strings like a parachute. These strings are called chordae tendineae and are attached to the muscles on the ventricle walls. The pulmonary and aortic semilunar 
valves do not have chordae tendineae but still operate based on pressure gradients, each using three pocket-like cusps that inflate when the pressure rises. (Marieb, 1995)

\subsubsection{Heart Disease}

Congenital, valvular, ischemic, viral and idiopathic are types of heart disease that contribute to congestive heart failure. Congestive heart failure is the term used to describe the condition where the heart muscle is continuously weakening. The weakening of the heart may be due to coronary atherosclerosis, multiple myocardial infarcts, dilated cardiomyopathy or high blood pressure. (Braunwald, 1992)

Coronary atherosclerosis is the blockage of the vessels in the heart which results in a lack of oxygenated blood arriving at the heart muscle. This often results in the death of heart muscle leading to myocardial infarcts. (Braunwald, 1992)

Myocardial infarcts are small heart attacks that kill off small parts of the heart muscle. The dead tissue is replaced with fibrous scar tissue which weakens the heart and disrupts the signals telling the heart when to contract. (Braunwald, 1992)

Dilated cardiomyopathy is caused when the heart muscle stretches and weakens resulting in poor ventricular contractility. High blood pressure resists the flow of blood from the heart which forces the heart to work much harder to maintain blood circulation. This progressively weakens the heart. (Braunwald, 1992) 
All of these problems result in reduced performance of the heart and reduced circulation of blood. Some signs of heart disease include fatigue, pulmonary edema (fluid in the lungs) and edema (pooling of blood in feet, ankles and fingers). (Braunwald, 1992)

Heart disease in early stages can be treated by reducing the load on the heart by giving it a chance to recuperate. This can be done with digoxin (forces the heart to beat stronger), by reducing fluid in circulation with diuretics (drugs which force the body to excrete sodium and water in the urine) and by reducing blood pressure with ACE (Angiotensin Converting Enzyme) inhibitors. Ventricular assist devices can also be used on a short term basis to reduce the load on the heart and give it time to heal. (Marieb, 1995)

Heart disease in its final stages can only be treated with a heart transplant where the heart is surgically removed and a donor heart is implanted in its place. This treatment is not without problems. Host bodies naturally try to reject the foreign heart. Immune suppressant drugs are used to encourage the host body to accept the new tissue, but these drugs severely depress the host's resistance to fungal, viral, and bacterial infections. Immune suppressant drugs also have side effects which cause severe bone marrow depression, enlargement and reduction of red blood cells and rheumatoid arthritis. Other serious side effects include skin cancer and lymphomatous tumors. These problems often result in reduced quality of life for many transplant patients. (Barnhart, 1990)

\subsubsection{Blood Vessels and Blood Pressure}

Blood is carried throughout the body in blood vessels. The heart pumps blood out through arteries and blood returns to the heart in veins (see Figure 4). Capillaries connect the arteries to the veins. In the systemic system, blood is de-oxygenated as it passes 
through the capillaries and feeds the surrounding tissue. In the pulmonary system, the capillaries are used to oxygenate the blood. Since arteries always leave the heart, the pulmonary arteries carry de-oxygenated blood while the pulmonary veins carry oxygenated blood. For the systemic system, this is reversed: the arteries carry oxygenated blood while the veins carry de-oxygenated blood. (Marieb, 1995)

As the heart pumps blood out through the aorta, a pressure surge occurs. In the average person, this pressure varies from 80 to $120 \mathrm{mmHg}$. These pressures can be measured on the arm but are taken as the pressure leaving the heart directly into the aorta. As the blood moves through the systemic system, the blood pressure decreases to $30 \mathrm{mmHg}$ in the capillaries and 1 to $5 \mathrm{mmHg}$ in the venae cavae. In the pulmonary system, the pulmonary artery has pressures varying from 10 to $25 \mathrm{mmHg}$, and the pulmonary veins have pressures varying from 1 to $5 \mathrm{mmHg}$. (Marieb, 1995)

\subsection{Artificial Hearts \& Mechanical Assist Devices}

Originally, in the 1950 s, mechanical circulatory assist devices were planned as permanent replacements for hearts in end-stage heart disease patients. Experiences with the Jarvik heart in 1982 showed that this was not a simple task with the technology of the time (Hill, 1993). As a result, research focused on employing VADs as a bridge to transplant or temporary support with permanent replacements as the ultimate goal. Bridge to transplant implies keeping the patient alive until a donor heart becomes available. Experience with bridge to transplants has given researchers the opportunity to study several problems that prevent the use of mechanical devices for permanent replacement of the biological heart. (Rowles et. al., 1993) 
Using artificial devices as permanent solutions to heart disease is an alternative to transplantation. It is difficult to compare an artificial heart with a biological heart, since an artificial device will never be as reliable or easy to maintain as the original heart. However, there are advantages of artificial devices over transplanted hearts. Artificial devices do not require the use of immune suppressant drugs which is a significant advantage of mechanical devices over heart transplants. Other advantages include lower costs and higher availability. (HSVAD Product Literature, 1997)

A Total Artificial Heart (TAH) works as a replacement for the biological heart of a patient. When TAHs are implanted, the biological heart is completely removed. Mechanical assist devices are used to help the biological heart pump blood throughout the body. These devices have many names including Electrohydraulic Ventricular Assist Device (EVAD), Left Ventricular Assist Device (LVAD) or simply Ventricular Assist Device (VAD). Some mechanical assist devices may be used on the left, right or both sides of the patient's heart. These devices are usually used to assist the left ventricle since this is the part of the heart that does not pump sufficient blood after heart disease has set in. (Rowles et. al., 1993)

\subsubsection{Technical Challenges}

There are many problems that must be addressed in the design of an artificial heart. Thromboembolism (blood clots), anatomical fit, sepsis (infection), multiple organ failure, quality of life (tethered to a large console), reliability, durability, noise reduction, vibration, high efficiency, reaction forces on adjacent tissue, low heat dissipation, high efficiency, control, communication, minimal maintenance and monitoring. (Diegel et. al., 1992) 


\subsubsection{Types \& Manufacturers}

There are many different types of artificial hearts: pneumatically-actuated pulsatile pumps, electrohydraulic pulsatile pumps, non-pulsatile centrifugal pumps, pusher plate pulsatile pumps and roller pumps. All of these devices have advantages and disadvantages and their manufacturers are distributed in many places around the world. (Macoviak et. al., 1990)

In North America, there are six devices in particular that are experiencing a level of success. These devices are: Abiomed TAH, Abiomed BVS-5000, Novacor LVAD, Thoratec BI-VAD, Heartmate LVAD and Biomedicus Centrifugal Pump. All of these devices fall into two different classes of devices; continuous flow and pulsatile pumps. (Rowles et. al., 1993)

Continuous flow pumps maintain a steady flow of blood through the device. There are two types of continuous flow devices, roller pumps and centrifugal pumps. Roller pumps propel the blood by squeezing it through a tube using rollers. Centrifugal pumps develop a flow vortex which uses centrifugal force to pump the blood. These devices are simple and effective for short-term use; however, they are not suitable for long-term support due to their non-pulsatile nature and because of the damage they cause to the patients blood.

Pulsatile pumps can be grouped into three groups: pusher plate, pneumatic and hydraulic. These devices all use a polymer diaphragm which is displaced in a blood chamber. This squeezing action is similar in function to the left ventricle. As the diaphragm moves back and forth, iniet and outlet valves open and close to prevent back-flow of blood. These 
devices function in a manner similar to the natural, biological heart. Pulsatile devices are much more complicated, costly and difficult to develop than continuous flow devices.

The major difference between pulsatile devices is in the method used to displace the flexible diaphragm. Pusher plate pumps have a metal disk that displaces the polymer diaphragm. The Novacor LVAD is an example of a pusher plate pump. When the device is implanted, an air vent must be run from the pusher plate side of the diaphragm out through the patient's skin. If this is not done, an air vacuum develops inside of the device that resists the motion of the pusher plate. As a result, the stroke volume and efficiency of the device are reduced. Piercing a patient's skin creates a significant risk for infection and reduces the probability for success of long-term implants. (Macoviak et. al., 1990)

Pneumatic devices displace the diaphragms by pumping air from a compressor into the device. These devices are not totally implanted as the tubes that are required to pump air in and out of the device must be passed through the patient's skin. The air compressors on these devices are large and bulky, and when combined with tubes piercing the skin, these devices are unsuitable for long-term implants. (Rowles et. al., 1993)

Hydraulic devices use a hydraulic fluid, such as silicone oil, to displace the pumping diaphragm. Since these devices do not use a gas to displace the diaphragm, they avoid the problems associated with the high permeability rates of gases through the polymer diaphragms. Unfortunately, flexible polymers are naturally highly permeable materiais compared to glass, ceramics and metals and the loss of silicone oil through the diaphragm is a possibility. This thesis investigates this problem. 


\subsubsection{HeartSaver Ventricular Assist Device (HSVAD)}

The HSVAD is a pulsatile ventricular assist device. It is capable of being remotely powered, controlled and monitored. Unlike many other devices, it is implanted above the diaphragm in the chest cavity. The HSVAD has been designed for permanent, long-term circulatory support but may be used as a short-term bridge to transplant. After being implanted, it requires no openings in the skin for power transmission, monitoring or venting of the device. The goal of the HSVAD is to allow a patient to receive ongoing circulatory support while carrying on life in as normal a fashion as possible. (HSVAD Product Literature, 1997)

The HSVAD in its current design is an LVAD. When implanted, it is located in the chest cavity above the diaphragm (see Figure 5). It is designed to assist the left ventricle in pumping blood. It is attached to the base of the left ventricle using an inflow cannula (see Figure 6). Blood is ejected from the device through an outflow cannula into the ascending aorta. The two artificial valves used with the device are located in the ends of the cannulas. (HSVAD Product Literature, 1997)

The device is designed to pump at the same rate as the natural heart. During diastole (relaxation of the heart), the natural heart fills with blood and passes it through the left ventricle into the device. The device fills passively and detects when it is full through the use of Hall effect sensors located on the unit's shell. When a full signal is received, the device pumps the blood, raising the pressure in the blood chamber. When the pressure in the blood chamber rises above the pressure in the inflow cannula, the inflow valve closes. Then, when the pressure in the ventricle exceeds the back-pressure in the outflow 
cannula, the outflow valve opens allowing the blood in the VAD to be ejected. The Hall effect sensor detects when the stroke is complete and pumping stops which results in lowered pressure in the blood chamber. When the pressure in the blood chamber drops below the back pressure in the outflow cannula, the outflow valve automatically closes. As the pressure continues to drop below the pressure in the inflow cannula the inflow valve opens. When the inflow valve opens, the blood starts flowing into the blood chamber under its own pressure gradient until the Hall effect sensor detects full-fill and the pumping cycle begins again. (King, 1996)

The HSVAD is an electro-hydraulic device. It has two chambers, a blood chamber and a volume displacement chamber (VDC) (see Figure 7). The blood chamber is divided into two parts separated by a flexible polymer diaphragm called the blood chamber diaphragm (trade name Biospan, segmented polyether-polyurethane) (see Figure 8). The two chambers are separated by three components, a polyurethane bulkhead, an energy converter and a passive fill valve (see Figure 9). The VDC is sealed using another flexible diaphragm made of the same material as the blood chamber (see Figure 10). The VDC and half of the blood chamber (the space between the blood diaphragm and the bulkhead) are filled with the hydraulic fluid, silicone oil (decamethyltetrasiloxane). (King, 1996)

The blood chamber diaphragm has a magnet located at its center (see Figure 8) and the polyurethane shell has Hall effect sensors located on both sides of the shell to detect the proximity of the diaphragm to the shell surface. When the device fills naturally from pressure in the venous system, the blood flows into the device and displaces the blood chamber diaphragm. This pushes silicone oil in the oil side of the blood chamber out 
through the passive fill valve and energy converter into the VDC. When the magnet in the diaphragm gets close enough to the Hall effect sensor, it sends a full-fill signal which starts the energy convertor pumping. The energy convertor drives silicone oil from the VDC into the silicone oil side of the blood chamber at a very high rate. When the silicone oil pressure in the blood chamber rises to a certain level above the pressure in the VDC, a spring in the passive fill valve compresses allowing the passive fill valve to close. As the silicone oil pressure rises in the blood chamber, the flexible diaphragm displaces the blood from the blood chamber out through the outflow cannula. This continues until the magnet in the blood diaphragm gets close enough to the Hall effect sensor to send a full-eject signal that turns off the energy convertor. When the energy convertor turns off, the passive fill valve opens and pressure from the inflowing blood drives the silicone oil back into the VDC until the cycle begins again. (King, 1996)

The use of silicone oil is one of many ingenuities that make the HSVAD one of the best designs in the world. The silicone oil works as hydraulic fluid to displace the diaphragm, as lubricant for motor bearings, as coolant for circuits in the device and thus avoids venting problems associated with using pneumatic devices. As a result, the device is totally implantable. Unfortunately, biocompatible polyurethanes which are suitable for use as blood contacting surfaces are typically permeable materials. This is an inherent quality associated with flexible and thin polyurethane membranes.

\subsection{Blood}

Blood is the transport medium that carries oxygen, nutrients, wastes, hormones and any other components that need to be transported around the human body. Blood is 
considered as a form of connective tissue yet it has no collagen or elastic fibers. As a result, it is a fluid. Both liquids and solids make up the constituents of blood. Blood is made up of many components including water, red blood cells and white blood cells. When blood is spun in a centrifuge, these components will separate out into 3 layers. The least dense (top) layer is plasma which makes up $55 \%$ of whole blood. $90 \%$ of plasma is water and the remaining $10 \%$ is dissolved solutes including proteins, acids, salts, enzymes, nutrients, electrolytes and respiratory gases. The next layer is the buffy coat. This layer makes up less than $1 \%$ of the whole blood volume and contains the platelets and white blood cells. The bottom layer is red blood cells which make up the remaining $45 \%$ of the blood volume. (Marieb, 1995)

An adult human has approximately $5 \mathrm{~L}$ of blood which circulates through arteries, capillaries and veins. Blood that has been oxygenated after moving through the lungs is a scarlet colour due to its high oxygen content. The blood that is returning to the lungs is oxygen poor and appears dark red. The pH of blood varies between 7.35 and 7.45 and it maintains a temperature slightly higher than body temperature at $38^{\circ} \mathrm{C}$. (Marieb, 1995)

Blood has several functions including distribution, regulation and protection. These functions work together using blood as a medium to maintain efficient operation of the main systems in the human body. The distribution system transports oxygen and nutrients from the lungs throughout the body. Blood also transports nitrogenous wastes and carbon dioxide to waste disposal sites like the lungs and kidneys. The transport of hormones between organs is also performed by blood. Body temperature is regulated by absorbing and distributing heat throughout the body. The $\mathrm{pH}$ is maintained through using proteins and other blood components as buffers. The volume of fluid in the circulatory 
system is also maintained using proteins and salts. Blood helps protect the body by circulating platelets and proteins which initiate clot formation and prevent blood loss. The blood carries antibodies and white blood cells that fight bacteria and viruses. (Marieb, 1995)

Red blood cells (RBC) or erythrocytes, transport oxygen and carbon dioxide by passing the cells through the lungs where oxygen permeates into the blood. The oxygen is then drawn out of the blood into the red blood cells where the oxygen binds to iron atoms in the hemoglobin. When the $\mathrm{RBC}$ reach tissues needing oxygen, the process is reversed and the oxygen leaves the RBC and diffuses into the tissue. The hemoglobin can then pick up carbon dioxide. $20 \%$ of the carbon dioxide carried in the blood is in hemoglobin. (Marieb, 1995)

Ideally, testing for permeability would use human blood as a receiving fluid. Unfortunately, the value placed on human blood and the procedures required to obtain permission were prohibitive. CVD/OHI had previously obtained permission to use animals for testing of the device. The testing for this thesis falls in this category and, as a result, the excess bovine blood that was available was used for tests in this thesis.

Bovine blood is similar to human blood; however, it is important to note that blood characteristics from either humans or bovines are influenced by age, sex, breed, training, sampling technique, environment, diet, time of day and season. (Williams et. al., 1995)

Table 1 shows that bovine blood is similar to human blood with the exception of the number red blood cells. 


\subsection{Biomaterials}

The major problem with the selection and design of biomaterials is their lack of resistance to reaction with blood. Possible reactions can occur when an artificial surface is introduced to blood, including the deposit of proteins and platelets on the surface resulting in the formation, growth and embolization of blood clots. Any material that is to be tested for long-term blood contacting applications must meet several requirements including:

1) no destruction of formed elements (blood cells) of the blood

2) no alteration of plasma proteins

3) does not cause thrombosis

4) no destruction of enzymes

5) no depletion of electrolytes

6) no adverse immune responses

7) does not cause cancer

8) does not cause toxic reactions

9) does not cause allergic reactions

10) does not damage tissue

Experience has shown that the most difficult requirement to satisfy is requirement (3). In the case of polyurethanes, most of the requirements are met; however, thrombosis is still a problem. From a material point of view, two strategies can be used to reduce thrombosis formation. The first is to use a material which is very smooth so that the blood has no opportunity to deposit proteins or platelets on the surface. The second strategy is to place small fibers on the blood-contacting surface which promote the 
growth of a thin layer of cells over the surface. This tissue layer is similar to the inner lining of blood vessels which is highly resistant to clot formation. (McMillin, 1987)

Other material problems include calcification which is the development of calcium phosphate mineralization on blood pump surfaces. It develops due to a combination of mechanical stress, cellular interactions, thermal effects, and physiologic or biochemical factors. Fatigue resistance is a major factor in reliability of the polymer diaphragms. Areas of high stress in the flexing diaphragm operate as focus points for calcification. Once a hard calcium deposit forms on the flexible diaphragm, it can promote failure since it operates as a point of stress concentration. (Comstock, 1982)

The human body is a very corrosive environment. Enzymes in the blood are very effective catalysts that break down molecules. Enzymes are particularly efficient at breaking ether bonds which are a major component in segmented polyetherpolyurethanes. (Comstock, 1982)

Polymers are typically very sensitive to manufacturing and casting conditions. Temperature, drying time, humidity, solution density, and many other conditions effect the reliability and reproducibility of quality components. Any small defect can provide proteins and platelets with the opportunity to adhere to the polymer surface and develop thrombosis or calcification. (Wesslen et. al., 1994) 


\subsubsection{Survey of Biomaterials}

Several polymer materials have been investigated for use as flexible blood chamber diaphragms in artificial hearts and VADs. Of these materials there is one which has had continued success since the early 1980s. This material is a segmented polyetherpolyurethane called Biomer. The manufacturers of Biomer were DuPont and Ethicon corporations. They terminated production of Biomer due to product liability concerns. As a result, several other manufacturers started developing materials that were very similar to Biomer. These materials are noted with '*' in Table 2. Table 2 is not intended to be a complete list of biocompatible elastomers but is provided to indicate the wide range of polymers that have been tested for implantable applications. To date, the most successful polymers are segmented polyether-polyurethanes like Biomer. There are many clones of this successful material which have been modified slightly and renamed. (McMillin, 1987)

\subsubsection{Segmented Polyether-Polyurethane (Biospan)}

The polymer of interest in this thesis is a segmented polyether-polyurethane with the trade name Biospan. The Biospan for this thesis was provided by The Polymer Technology Group Incorporated, Emeryville, CA. Biospan is an aromatic polyetherurethaneurea with soft segment of polytetramethyleneoxide and a hard segment of diphenylmethane diisocyanate and diamines (chain extender). A stabilizing additive package which consists of an antioxidant and copolymers of decyl methacrylate and diisopropylaminoethyl methacrylate are the remaining components. Biospan was designed to be a direct replacement for Biomer. Figure 12 is a diagram of the molecular 
structure of Biospan. The material characteristics of Biospan, provided by The Polymer Technologies Group Inc., are provided in Table 3. (Biospan Product Literature, 1996)

Biospan was supplied in a viscous fluid solution of dimethylacetamide (DMAC). Casting of the material into diaphragms was performed by pouring the viscous solution over a highly polished aluminum mold. The mold was then placed in a humidity-controlled oven at $40-60^{\circ} \mathrm{C}$ where the DMAC solvent is evaporated off. This process was repeated until the desired thickness of the diaphragm is achieved. For the blood chamber diaphragm, 4 to 5 coatings were required depending on the viscosity of the solution. (King, 1996)

\subsection{Hydraulic Fluid}

The fluid used to drive the polymer diaphragm in the EVAD had to meet several requirements including viscosity, stability with respect to shear stress from the energy convertor and biological stability for implantation. One fluid that has been found to be suitable for EVAD applications is decamethyltetrasiloxane.

The decamethyltetrasiloxane used in the HSVAD was provided by United Chemical Technology, Bristol, Pennsylvania. One major component of silicone oil is silicon. Silicon is normally found in the form of sand and quartz where it is silicon dioxide or silica. Silicones belong to a family of silicon oxide polymers whose compositions vary based on the length of the polymer and their organic side chains. 
As stated earlier, the silicone oil used in the HSVAD is decamethyltetrasiloxane. This is considered a short chained siloxane (see Figure 13). Short siloxane polymers like decamethyltetrasiloxane have a low viscosity where long chain polymers can be semisolid. Siloxanes can be cross-linked through a vulcanization process called fumarization which makes the polymer more solid.

Decamethyltetrasiloxane is an excellent polymer for implantation due to its resistance to react with chemicals and enzymes in biological systems. Unfortunately, polydimethylsiloxanes have a very low glass transition temperature $\left(-120^{\circ} \mathrm{C}\right)$ which means they have excellent segmental mobility resulting in a high degree of swelling when exposed to polymers which results in high permeation rates. (Rice et. al., 1988) 


\section{Chapter 3: Permeability \& Polymers}

Most amorphous polymers are permeable. Glass, ceramics and metals are in general not permeable. The basis for the difference in permeability of polymers versus other materials is in their basic molecular structures. Polymers are long-chained molecules. Often, these molecules are not highly organized and, as a result, small voids occur in between and along the side of the long molecules. Polymers also have a relatively high

level of molecular motion when compared to ceramics, glass and metals. (Comyn, 1985)

The process through which permeability occurs in polymers is started by an activated process where a concentration gradient drives a permeant from a point of high concentration (donor chamber) to a point of lower concentration (polymer surface). The permeant then diffuses throughout the polymer in an attempt to achieve a uniform distribution of the permeant in the polymer. When the permeant reaches the far side of the polymer, it desorbs onto the surface where it can be washed away by the receiving medium. This process is driven by a concentration gradient from the higher concentration in the polymer to the lower concentration on the receiving side of the polymer. The properties of the permeant and the polymer are the influencing factors in 
the system. Ionic charges and hydrophobic/hydrophilic natures of the materials can greatly affect the rates of permeation. (Comyn, 1985)

The diffusion of a permeant through a homogeneous polymer with no defects such as pores, bubbles or cracks can be visualized as a series of diffusion steps. Each step represents a potential barrier which the permeant must overcome to achieve a concentration equilibrium. The force required for the permeant to overcome the barrier is provided by the concentration gradient. (Comyn, 1985)

In the process of overcoming each barrier, a cooperative rearrangement of the polymer and the permeant must be made. In order for a barrier to be overcome a number of van der Waals bonds (electrostatic forces between molecules) or other cohesive forces must be broken to permit the rearrangement of the polymer structure. The amount of energy (activation energy) required to rearrange the polymer is based on the size and shape of the penetrating species. Permeating species with molecules that have long chains or crosslinks will permeate slower than species with short molecules or no cross-links. In cases with large permeating molecules, several barrier steps must be overcome to allow the full length of the permeant to pass through a distance equal to its length. (Comyn, 1985)

In addition to concentration gradient, other factors can influence the permeation rate. Any factor that helps create "holes" can increase the permeation rate. An increase in temperature can reduce the density of a polymer by making the molecules more active thereby increasing the size and number of "holes" large enough to allow the permeating species to diffuse through. (Comyn, 1985) 
For VAD applications, polymer membranes work as a barrier to both permeation of silicone oil into the blood of the patient and of blood parts, water and gases into the device. Not only must the diaphragm resist the permeation of silicone oil during use invivo, but it must also resist permeation during storage and sterilization. Any significant loss of silicone oil would require refilling of the device which is undesirable.

\subsection{Permeability Theory}

A standard set of coefficients are used to compare the permeability characteristics of polymers. The coefficients include the permeability coefficient $(\mathrm{P})$, the diffusion coefficient (D) and the solubility coefficient (S). To minimize permeability, it is desirable to have a low diffusion coefficient and a low solubility coefficient. (Comyn, 1985)

\subsubsection{Fick's Law}

Fick's first law of diffusion (Equation 1) is applicable for cases where the concentration in the donor chamber and receiving chamber does not change with time, i.e. steady state:

$$
F_{x}=-D\left(\frac{\partial c}{\partial x}\right)
$$

where

$F_{x}$ is the flux of permeant in the $x$ direction and $(\partial c / \partial x)$ is the concentration gradient (inside of the barrier) in the $\mathrm{x}$ direction. 
Fick's second law of diffusion is used for the non-steady state cases where the concentration of permeant in the donor chamber and receiving chamber change with time:

$$
\frac{\partial c}{\partial t}=D\left(\frac{\partial^{2} c}{\partial x^{2}}+\frac{\partial^{2} c}{\partial y^{2}}+\frac{\partial^{2} c}{\partial z^{2}}\right)
$$

When diffusion is only in the $\mathrm{x}$-direction, Equation 2 reduces to:

$$
\frac{\partial c}{\partial t}=D \frac{\partial^{2} c}{\partial x^{2}}
$$

Equation 3 is true for unidirectional diffusion in cases where the diffusion coefficient is constant and independent of $x, t$ or $c$. Frequently, $D$ is not constant but a function of concentration in polymer-permeant systems. The dependence of $\mathrm{D}$ on concentration is due to the plasticizing effect of absorbed permeant and mechanisms that immobilize portions of the absorbed permeant. Plasticization is the decrease in cohesive forces between polymer chains which results in increased mobility of the molecules. Naturally, this results in an increased rate of diffusion and lower activation energy for diffusion.

The problem presented in this thesis assumes a constant concentration gradient between the donor and receiving chambers once steady state conditions have been reached. This assumption is based on the following facts: The EVAD has two chambers separated by a non-porous membrane with $100 \%$ silicone oil on one side and circulating blood on the other side. The blood is constantly being filtered by organs in the human body, hence the concentration of silicone oil in the blood is expected to remain near zero. 
A simplified model of the membrane can be made using a film permeation model. In the film permeation model, a film of thickness $l$ separates two chambers. Initially both chambers are free of the permeant. At time $t=0$, the permeant is introduced to one chamber. The amount of permeant that passes through the membrane is shown in Equation 4 (Comyn 1985):

$$
\frac{Q}{l A C_{1}}=\frac{D t}{l^{2}}-\frac{1}{6}-\frac{2}{\pi^{2}} \sum_{n-1}^{\infty} \frac{(-1)^{n}}{n^{2}} \exp \left(-\frac{D n^{2} \pi^{2} t}{l^{2}}\right)
$$

where $C_{1}$ is the concentration at the permeant face of the membrane, $A$ is the area of permeation and $Q$ is the volume of permeant passing through the membrane. A plot of this equation (see Figure 14) shows a non-steady state period where the permeant builds up in the film. After this, a linear relationship develops which is steady state permeation. The steady state portion of the curve can be modeled by a simplified form of Equation 4:

$$
Q=\frac{D A C_{1}}{l}\left(t-\frac{l^{2}}{6 D}\right)
$$

From Figure 17 and Equation 5, we find the diffusion coefficient (D) through the use of the time lag method, where $\mathrm{Q}=0$. The time lag $(\tau)$ in Equation 6 is the intercept on the time axis using the straight portion of the curve, which can be used to find the diffusion coefficient (D).

$$
D=\frac{l^{2}}{6 \tau}
$$

As the silicone oil diffuses into the membrane, the establishment of a concentration gradient across the membrane will take some time and will therefore give rise to a lag 
effect. This can be seen in the curved region in Figure 17. Here the permeant is being absorbed and distributed throughout the polymer, (i.e. dc/dt is greater than 0 ). In the linear portion of the curve, the permeant has distributed itself evenly throughout the polymer, and the concentration in the polymer has become constant (i.e. dc/dt is equal to $0)$. Fick's first law can be applied.

The permeation coefficient $(\mathrm{P})$ can be determined from the linear portion of the slope in Figure 14.

$$
P=D S
$$

Equation 7 can be rearranged to solve for the solubility coefficient (S).

$$
S=\frac{P}{D}
$$

Fick's first law can now be used to derive an equation to predict the volume of permeant permeating through a polymer using the permeability coefficient.

$$
F_{x}=\frac{-D\left(C_{2}-C_{1}\right)}{l}
$$

The total volume (Q) of permeant passing through the film after time ( $t)$ is:

$$
Q=\frac{-D A t\left(C_{2}-C_{1}\right)}{l}
$$


where $C_{1}$ is the concentration of the permeant in the polymer at the donor surface, $C_{2}$ is the concentration of the permeant in the polymer at the receiving surface, $l$ is the thickness of the film, $t$ is time and $Q$ is the volume.

$C_{1}$ and $C_{2}$ are unknown but the concentration of the permeating species in the donor $\left(C_{d}\right)$ and receiving chambers $\left(\mathrm{C}_{\mathrm{r}}\right)$ are known and are related through the following equation.

$$
S=\frac{C_{1}}{C_{d}}
$$

where $\mathrm{S}$ is the distribution or partition coefficient.

Substituting Equation 10 into Equation 9 we get:

$$
Q=\frac{-D S A t\left(C_{r}-C_{d}\right)}{l}
$$

Substituting Equation 7 into Equation 11 and using our assumption of $C_{r}=0$ we have:

$$
Q=\frac{P A t\left(C_{d}\right)}{l}
$$

The volume of permeant permeating though a polymer can now be predicted.

\subsubsection{Diffusion into a Thin Film}

One popular method for determining the permeability characteristics of a polymer is to test a thin specimen of constant thickness by soaking it in a liquid. By sampling the uptake of fluid into the polymer, the diffusion coefficient can be determined. Equations 
that model these systems have been derived from Fick's second law. The following equation was derived by Comyn, (1985).

$$
\frac{C}{C_{1}}=1-\frac{4}{\pi} \sum_{n=0}^{\infty} \frac{(-1)^{n}}{2 n+1} \exp \left(\frac{-D(2 n+1)^{2} \pi^{2} t}{4 l^{2}}\right) \cos \left(\frac{(2 n+1) \pi x}{2 l}\right)
$$

The film is of thickness $2 l$, its faces being located at $+l$ and $-l$ and $\mathrm{x}=0$ at the center of the film. $C_{1}$ is the concentration at the faces of the film, which are established instantaneously. The effects of diffusion at the edges of the film are not taken into account. Equation 13 can be integrated to yield Equation 14 which gives the mass uptake by the film, $M_{r}$.

$$
\frac{M_{t}}{M_{f}}=1-\sum_{n=0}^{\infty} \frac{8}{(2 n+1)^{2} \pi^{2}} \exp \left(\frac{-D(2 n+1)^{2} \pi^{2} t}{l^{2}}\right)
$$

$\mathrm{M}_{\mathrm{f}}$ is the mass uptake at equilibrium, and $l$ is again film thickness. At short times, this equation reduces to:

$$
\frac{M_{t}}{M_{f}}=\frac{4}{l}\left(\frac{D t}{\pi}\right)^{1 / 2}
$$

which provides a convenient way of measuring $D$ from mass uptake experiments.

Research performed by Gent and Tobias (Gent et. al., 1982) shows that polydimethylsiloxane elastomers absorb silicone oil up to an equilibrium amount ranging from $10 \%$ to $50 \%$. Equation 15 is true for cases in this range which are linear, and a permeant is absorbed by a diffusion process and is not associated by chemical changes in the elastomer. 
The diffusion constant takes into account the effects of structure, morphology and all other factors affecting transport. Since it is currently impossible to mathematically predict all of these effects, the diffusion coefficient (D) is found experimentally.

\subsubsection{External Factors Affecting Permeability}

The permeability of a polymer-permeant system can be affected in many ways. Some of these factors include stress on the polymer, flexing of the polymer, temperature, boundary conditions, and environmental effects. These factors can work to increase or decrease the permeation rate of a permeant through a polymer. (Comyn, 1985)

The permeation rate or diffusion of a permeant through a polymer is often modeled by assuming there are "holes" which the permeating species must pass through. The effect of external factors on permeation through a polymer can be considered by examining the effect of any given factor on the hole size or energy required to drive the permeating species through the polymer. (Comyn, 1985)

When stress is applied to a polymer, it rearranges the molecules in the polymer in the direction of the applied force. If the applied stress is in the direction of permeation (high to low gradient), then the permeation rate may increase due to the formation of linear pathways that are created between molecules as they straighten. Stress can also induce a decrease in permeation rate since the stress will also draw the molecules closer together (Poisson's effect). Applied stress may also increase the energy required to displace two adjacent molecules since the molecules are under a tension force. Increasing the 
activation energy for permeation will also reduce the permeability rate. The net effect of stress is that the size of the holes decreases and the energy required to displace molecules increases, resulting in a reduced volume of absorbed permeant which may result in reduced permeability. It is also important to note that the thickness of the polymer also decreases, thereby reducing the distance that the permeant molecule must travel which may increase the permeability of the polymer. (Comyn, 1985)

Flexing of the polymer is a mechanical manipulation of the molecules in the polymer. Forcing the molecules to move decreases the energy required for the permeating species to pass through the polymer. An increase in the degree of aggressiveness in flexing the polymer may result in increased permeation rates. (Comyn, 1985)

Increases in temperature activate the polymer molecules resulting in voids through the movement of polymer segments which can be occupied by the diffusing species. As a result, the diffusion coefficient will increase with rising temperature. Permeability in a polymer is highly dependent on temperature, particularly at levels near the polymer's glass transition temperature. Polymers typically have higher permeability when they are in a rubbery state. (Comyn, 1985)

The boundary conditions on the surface of a polymer in a diffusion system can have a significant effect on permeation rate. If the degree of agitation of the fluid on a membrane surface is stagnant, it may function as an additional resistance to mass transfer. This effect can be simulated using a simple resistance model where there are new layers on either side of the membrane surface (see Figure 15). (Comyn, 1985) 
The total diffusion resistance $\left(r_{\text {total }}\right)$ can be calculated by the sum of the resistance in the polymer membrane $\left(r_{p}\right)$, the resistance of the donor chamber $\left(r_{d}\right)$ and the resistance of the receiving chamber $\left(r_{\mathrm{r}}\right)$. (Comyn, 1985)

$$
r_{\text {total }}=r_{d}+r_{p}+r_{r}
$$

In cases where the flow over the membrane is turbulent, the boundary layer effects can be neglected. It is, however, important to consider that in the case of this thesis, proteins and platelets may adhere to the membrane surface creating additional layers. The environmental effects specific to this thesis include the adhesion, and in some cases, growth of blood parts into the diaphragm surface. Aggressive environments can also have serious corrosive effects, especially when considering the effects of enzymes destroying ether bonds in polyether-polyurethanes. (Iordanskii, 1994)

\subsubsection{Polymer Characteristics Affecting Permeation}

The composition, structure and state of a polymer controls the diffusion rate of a permeant. Some characteristics that are used to control the permeability of a polymer include thickness, molecule polarity, crystallinity, density, additives and degree of organization of the polymer. (Comyn, 1985)

The permeation rate of a permeant diffusing through a polymer is inversely proportional to the thickness of the polymer sample. Increasing the thickness of a membrane will reduce the permeation rate due to the increase in the diffusion path length. The use of 
polar molecules in a polymer can also reduce the permeation rate. Polar molecules can resist the absorption of other molecules. (Comyn, 1985)

The degree of crystallinity and organization of a polymer affect the permeability in the same way. By increasing the crystallinity or degree of organization in crystalline or amorphous polymers, the density is increased. This results in smaller spaces available for the permeating species and higher activation energies. The permeability of an amorphous polymer below its glass transition temperature is dependent on the degree of orientation of the molecular segments. (Comyn, 1985)

Crystals are typically impermeable to diffusing species. As a result, they form islands throughout a polymer which resist the permeating flux. It has been found that solubility is proportional to the amorphous volume fraction. (Comyn, 1985). In the diffusion process, the impermeable regions require permeant migration around them which increases the average path length relative to the nominal dimensions of the membrane.

"The crosslinking action of crystalline regions on sorption and transport is based on the effects of crosslinking on those processes in non-crystalline polymers. In those systems, diffusion coefficients decrease approximately linearly with crosslink density or the reciprocal of the molecular weight between crosslinks at low to moderate degrees of crosslinking. At higher densities the decrease is nonlinear. The solubility is relatively unaffected except at very high degrees of crosslinking or when the permeant significantly swells the polymer. 
The restraints of crosslinking on the segmental mobility of the polymer make the diffusion process more dependent on the size and shape of the permeant molecule. Thus, the selectivity of the polymer membrane is enhanced in agreement with the generally observed correlation of increased selectivity with a decrease in diffusion rate from almost any cause. Likewise, the decrease in inherent segmental mobility makes the diffusion process more concentration dependent.

Orientation of polymer materials introduces an asymmetric structure such that diffusion rates vary relative to the axis of orientation. Generally, the rate of penetration parallel to the axis is less than the rate in a randomly oriented sample which is less than the rate perpendicular to the axis. The validity of this generalization is dependent, in part, on the homogeneity of orientation. The generation or disappearance of voids during orientation can affect subsequent transport processes." (Comyn, 1985)

Additives and fillers can be used to either increase or decrease the permeation rate of a polymer membrane. If the correct fillers are used, the diffusion path through the polymer can be increased which will reduce the diffusion coefficient. If the filler used is impermeable to the diffusing species, the volume of permeant absorbed will be reduced which reduces the sorption coefficient. The combination of these two effects can greatly reduce the permeability of a polymer. Plasticizers usually result in an increased permeation rates due to the decreased cohesive forces between the chains, resulting in higher segmental mobility. (Comyn, 1985) 
The permeability characteristics of a polymer can be improved (i.e. decreased) by increasing the degree of crystallinity, introducing suitable fillers, coating the surface of the polymer or laminating the polymer with several layers. (Comyn, 1985)

\subsubsection{Permeating Species Characteristics Affecting Permeation}

The permeation rate through a polymer is controlled by the molecular structure of the material. The molecular structure of the permeating species is also an important factor in the permeation rate of the species through the polymer matrix. Permeant characteristics which affect permeability include molecule size, shape, polarity, and similarity to polymer membrane. (Comyn, 1985)

Small molecules permeate faster than large molecules due to the lower activation energies required for the permeating molecule to be forced through the membrane molecules. Molecules with large branches due to cross-linking will permeate slower through a polymer membrane due to difficulties driving the branches in between the membranes molecules. A highly cross-linked permeating species has a higher activation energy for permeation. Typically polar molecules permeate slower than non polar molecules. (Comyn, 1985)

The similarity of a polymer to the permeating species also affects the permeation rate with similar permeant/polymer cases having higher permeation rates. When the permeant and polymer membranes are similar, the polymer will contain functional groups which are similar to those of the diffusing species. In these cases, cohesive forces between the permeant and polymer membrane molecules are greater than the permeant-permeant 
interactive forces, and the membrane will absorb a substantial volume of the permeant. This results in an increase in the permeability constant because of the high concentration of permeant in the membrane which loosens the polymer network allowing easier diffusion and a higher diffusion coefficient. (Comyn, 1985)

When a permeant and membrane are dissimilar, the interactive forces between the membrane and permeant will be less than the cohesive forces between two permeant molecules and will tend to separate and form clusters. (Comyn, 1985)

\subsection{Literature Review - Permeability/Diffusion/Sorption}

The permeation of silicone oil through a segmented polyether-polyurethane into blood is a very specific problem which has not been extensively studied. Two published papers and three reports have been written for $\mathrm{CVD} / \mathrm{OHI}$ which have attempted to model the problem using water and saline as the receiving fluid. Other papers have only examined the permeation of gases and water vapour through segmented polyether-polyurethanes into a gas on the receiving side of the membrane.

Two of the published papers written by Matsuo et. al., $(1991,1992)$ studied the permeation of silicone oil through a Pellethane diaphragm into water using inductively coupled plasma atomic emission spectrophotometry. Two reports were written at the University of Utah by Pauley et. al., $(1990,1991)$ which attempted to determine the diffusion of silicone oil through Biomer into water using radio-labeling. One confidential report was prepared for $\mathrm{CVD} / \mathrm{OHI}$ which attempted to investigate the permeation of silicone oil through segmented polyether-polyurethane diaphragms. 
The permeation of water vapour through the diaphragms is a problem that has been considered in some papers. This problem is significant since water vapour inside the devices may promote corrosion of the internal components and electronics. Other papers examining the permeability of segmented polyether-polyurethanes focused on the permeability of gases through the polymer.

The following sections summarize the importance of the findings and detail the impacts of each paper individually.

\subsubsection{Silicone Oil Permeation}

Matsuo et. al., (1991, 1992)

Matsuo et. al. were investigating the permeation of silicone oil through the pumping diaphragm made of Pellethane (a segmented polyurethane) into the circulatory system. In-vitro experiments were used to determine the permeability of the diaphragm to silicone oil. The tests showed that the Pellethane was permeable to silicone oil. A permeation rate (specifically for their device) of $2.4 \mathrm{ml}$ year was stated in the 1991 report and a rate of $1.75 \mathrm{ml}$ /year was stated in the 1992 report. The diaphragm measured $.45 \mathrm{~mm}$ thick and had an area of $55.7 \mathrm{~cm}^{2}$.

Two types of in-vitro tests were performed to determine the permeability characteristics of Pellethane. A soaking test was performed to determine if the Pellethane sheet was indeed permeable to silicone oil. A diffusion test was performed to measure the permeability of Pellethane to silicone oil into water. 
The soaking test used several Pellethane samples with an area of $1.0 \mathrm{~cm}^{2}$ and a thickness of $.45 \mathrm{~mm}$. The samples were completely submerged in silicone oil for durations up to 14 days. When a sample was removed, it was washed using ethanol to remove adherent silicone oil and then dissolved in dimethylformamide (DMF). The volume of silicone oil in each sheet was determined by testing the DMF samples with gas chromatography.

The results from the soaking test showed that the Pellethane diaphragms became saturated with silicone oil at a duration of 3 days, as stated in the conclusion of the report. However, the graph included with the paper indicates that from the third day to the fourteenth day, the mass of silicone oil detected in the sheets increased from $.288 \mathrm{mg}$ to $.362 \mathrm{mg}$, which is obviously not constant. No calculations were made to determine the diffusion, solubility or permeability coefficients of silicone oil into Pellethane. The graph indicated that the Pellethane sheets with a thickness of $.45 \mathrm{~mm}$ and area of $1.0 \mathrm{~cm}^{2}$ absorbed up to $.362 \mathrm{mg}$ of silicone oil. No indication of the mass of the sheets was given, hence no calculation for the solubility can be made.

The diffusion test used a two chamber acrylic diffusion apparatus to determine the permeability coefficient of Pellethane to silicone oil. One side of the chamber contained $40 \mathrm{ml}$ of silicone oil while the other side held $40 \mathrm{ml}$ of plasma or distilled water. The chambers were separated by a Pellethane membrane with a thickness of $.45 \mathrm{~mm}$ and area of $3.14 \mathrm{~cm}^{2}$. The volume of silicone oil that permeated through the diaphragm into the receiving chamber was measured using inductively coupled plasma atomic emission spectrophotometry. Both the total bulk volume of the sample and the fluid used to wash the walls of the receiving chamber were tested using this method. 
The results from this experiment showed that silicone oil permeates through the Pellethane diaphragm into distilled water and plasma. This test shows that steady state permeation was reached at 3 days. However, the number of data points that were tested appears to be limited: water (4) and plasma (6). More data points would undoubtedly give a clearer picture of what was happening in the experiment. A comparison of the data from the water and plasma tests shows that the solubility of silicone oil in water and plasma is vastly different. It is interesting to note that whole blood was also not tested to determine if the solubility of silicone oil in whole blood is close to that of plasma. Obtaining information on the permeability of silicone oil into water is not useful since it does not model the conditions of an artificial device implanted in a human.

This paper concludes a permeation rate of $1.75 \mathrm{ml}$ year through a Pellethane diaphragm measuring $3.14 \mathrm{~cm}^{2}$ and a thickness of $.45 \mathrm{~mm}$. This conclusion was based on three data points at the third, fifth and seventh days of testing.

In order to obtain accurate results that will closely model the conditions found in the human body, the model must take into account as many variables as possible. This paper attempts to do this by using plasma in place of distilled water as a receiving fluid. It is suspected that blood would be a better receiving fluid than plasma since it has more lipophilic components that will attract and cluster with the hydrophobic silicone oil. This paper used Inductively Coupled Plasma Atomic Emission Spectrophotometer to analyze the plasma samples. This method breaks the sample down and measures the number of atoms of each component. This means that the test will count the total number of silicon atoms in a sample but not the number of decamethyltetrasiloxane molecules. A test method that measures the number of molecules not the total number of atoms may give 
more accurate results on the permeability, especially when the atoms in question are as common as silicon. Silicon is naturally found in blood. As a result, it could easily show up in the inductively coupled plasma atomic emission spectrophotometer results. This test has a lack of data points. The major conclusion of this paper is based on three data points.

An in-vivo test using goats was also reported in this paper. A ventricular assist device was attached to the circulatory system of the animals and was operated for a duration up to 21 days. Blood samples were taken and tested and the results showed that there were no changes in the serum silicon oil levels in the animals .

Pauley et. al., (1990, 1991)

Pauley and Ghanem attempted to model the permeation of silicone oil through Biomer into the human circulatory system using horizontal and vertical static diffusion cells at $37^{\circ} \mathrm{C}$. The donor fluid was decamethyltetrasiloxane and the receiving fluid was a saline solution. The method used to determine the volume of silicone oil permeating across the membrane was radio labelling. The diaphragms used in the test were $.178 \mathrm{~mm}$ thick Biomer samples. The LVAD which used these membranes actually incorporated 4 layers of Biomer separated by a micro-fine graphite lubricant.

When plotted, the results from this experiment did not follow Fick's laws. The discussion for the paper mentioned that the author was concerned that the test may have reached saturation of oil in water but no attempt was made to determine the saturation limit of silicone oil in water. 
This paper also reported on a swelling test where Biospan samples of thickness of 178 mm were submerged in silicone oil, saline and a 50/50 mixture of oil and saline. The paper reports that after 4 months, the mass of the samples never varied more than +-.005 grams.

The samples tested in this experiment are considerably thinner than the diaphragms used by CVD/OHI. The samples tested here were $.178 \mathrm{~mm}$ in thickness while the HSVAD uses diaphragms with thickness of approximately $.56 \mathrm{~mm}$. Pauley et. al., $(1990,1991)$ suggested that the diaphragms were porous. This may be true for their diaphragms since they were cast differently and were much thinner than the diaphragms cast at CVD/OHI which are considered non-porous.

Some of the conclusions from this experiment were that the rate of diffusion of silicone oil decreases with time and contact of silicone oil with Biomer affects its characteristics by reducing the permeation rate of silicone oil. The rate of permeation of oil through a diaphragm of $60 \mathrm{~cm}^{2}$ and thickness of $.178 \mathrm{~mm}$ was $20.5 \mathrm{~mL} / \mathrm{year}$.

The follow-up study showed that there were impurities in the silicone oil which permeate through the diaphragm faster than silicone oil molecules. The results from this experiment were incorrect. The first experiment had a permeability coefficient of $1.8 \times 10^{-7} \mathrm{~cm} / \mathrm{s}$, and when impurities were removed from the oil the permeability coefficient reduced to $2.2 \times 10^{-8} \mathrm{~cm} / \mathrm{s}$. While the second set of data are clearly more accurate than the first, it is still unknown what the presence of impurities is in the "cleaned" oil. If the presence of impurities is still high, then the second set of data would be only as useful as the first set. 
The follow-up study analyzed the permeability of several other radio-labelled model permeants through Biospan in an attempt to find a correlation between molecular weight and diffusion rate for polar molecules. It was suggested that micro-pores with diameters less than $10 \AA$ may allow small molecules to permeate quickly. It was also found that the diffusion of hydrophobic molecules (silicone oil) is not highly dependent on molecular weight. Biomer is more selective to hydrophobic than hydrophilic molecules. A recommendation was therefore made to replace decamethyltetrasiloxane with an oil which has hydrogen or oxygen replacing the methyl groups.

This report also stated that the solubility of silicone oil in water was found to be $4.2 \times 10^{-4}$ $\mathrm{mg} / \mathrm{ml}$ and therefore can be considered insoluble in water. Despite this fact the report continued to attempt to find the permeability of silicone oil through Biospan into water.

\subsubsection{Water Vapour/Gas Permeation}

The permeation of water and gases through the flexible polymers used in VADs has been considered in several papers. The permeation rate of gases can be quite high due to the small size of the gas molecules. In the case where air is used to pump the diaphragms, a loss of air from inside the device will result in reduced stroke volumes since there will not be enough air to fully displace the blood diaphragm. Hence, the full volume of blood will not be ejected from the device. This implies that the device will need to be refilled with air. McMillan et, al., (1989) have shown that $30 \mathrm{cc}$ of air may be lost through the VDC diaphragm in 15 to 20 days resulting in required refills of air on a monthly basis. 
The other problem involves the permeation of water from the blood through the diaphragm into the device. This issue is a problem since water will promote and induce corrosion of circuits, wires and other components found inside the devices. Saline inside a device will also have adverse affects on fatigue life and mechanical wear of motor components. (Reid et. al, 1985)

Reid et. al., (1985) assembled a diffusion test cell where water flowed over one side of a Biomer diaphragm and a nitrogen carrier gas flowed over the other surface. This permitted continuous measurements of the water vapour permeating through the diaphragm. The test apparatus used an optical dew point sensor downstream of the diaphragm to measure the volume of water that permeated through the diaphragm. From the data obtained through many tests, the diffusion and permeability coefficients were determined for several samples of Biomer with varying thicknesses.

The method of transport of water vapour through the diaphragm was determined to be diffusion after testing changes in donor chamber hydrostatic pressure $(50-200 \mathrm{~mm} \mathrm{Hg})$ were made. This indicates that bulk flow is not a transport mechanism in Biomer membranes. The results from this experiment concluded that Biomer is permeable to water and a sample $.366 \mathrm{~mm}$ thick has permeability, diffusion and partition coefficients of $3.2 \times 10^{-4}, 2.14 \times 10^{-7}$ and 1485 respectively.

Yeh et. al., (1994) recognized problems with moisture permeation and continued the research of Reid et. al., (1985). Attempts have been made to reduce the permeability of the diaphragms by creating trilaminate structures. These are sandwich polymer castings where the biomaterial (Biomer) is on the outside surfaces and a polymer which is highly 
resistant to gas/moisture permeation is located in between. The testing method to determine the permeability of these diaphragms to water is similar to the method used by Reid et. al., (1985).

Other researchers have come up with methods to test the permeability characteristics of segmented polyether-polyurethanes to moisture. Ramasamy et. al., (1989) placed humidity and pressure detectors in the volume compensator of a device and performed a bovine implant. The pressure and relative humidity in the compliance chamber were monitored to determine the amount of moisture permeation.

Yang et. al., (1996) used a glass capillary flow meter on the donor side of a sealed test chamber to determine the volume of water that permeated across the polyurethane diaphragm. As water was lost out of the donor chamber into the receiver compartment, the air bubble in the capillary flow meter would move. Once the diaphragm had been initially displaced, the bubble moved at a constant rate indicating permeation of water through the diaphragm. 


\section{Chapter 4: Material Testing}

There is some concern as to whether electrohydraulic devices using silicone oil as the driving fluid will lose some of the fluid over time. Based on the knowledge that most amorphous polymers are permeable materials, the question for this thesis is: How much silicone oil fluid will be lost through the blood chamber diaphragm of the device over 1 year? This question was interpreted as how much decamethyltetrasiloxane (silicone oil) will permeate through a segmented polyether-polyurethane diaphragm (Biospan) with a surface area of $60.5 \mathrm{~cm}^{2}$ and a thickness of $.60 \mathrm{~mm}$ in 1 year. Since it is difficult to model the diaphragm of an implanted VAD, it was decided to break the problem down into smaller manageable parts so that the influence of each factor that affects permeability could be analyzed. If the permeability results were high a strategy would be developed to reduce the permeability rate of the blood chamber diaphragms.

The first step in analyzing the permeability characteristics of a material was to select a test method that will provide values for the permeability (P), diffusion (D) and solubility (S) coefficients. There are many different units for these coefficients. The units selected for this thesis were based on the simple and frequently-used units of $\mathrm{cm}^{2} / \mathrm{s}$ for $\mathrm{P}$ and $\mathrm{D}$ and $g / g$ for $S$. 


\subsection{Candidate Test Methods}

There are several possible testing techniques available to determine the diffusion of a permeant through a polymer. Some possibilities include: volumetric, optical, radio-label or radio-trace, gas and vapour transmission, gravimetric techniques and diffusion cells.

The important factors to be considered for the acquisition of diffusion constants are:

1) Water cannot be effectively used as a receiving fluid due to silicone oil's highly hydrophobic nature. (Pauley et. al., 1991)

2) Blood should be used as the receiving fluid.

I) Blood has a limited availability.

II) Blood has formed elements (blood cells) that are held in suspension.

I) Blood must be stirred constantly; otherwise it will separate.

II) Silicone oil may not distribute evenly due to its hydrophobic nature.

3) A large number of samples must be taken to observe transient and steady state permeation rates.

4) Test method must be capable of detecting small silicone oil volumes in the microliter range.

5) Availability of polymer is limited.

6) The test must be carried out at body temperature. 


\section{Gravimetric Test Method}

The gravimetric technique determines the diffusion and solubility coefficients of a fluid in a polymer by weighing a polymer sample at regular intervals while the sample is submerged in a bath of the donor fluid. The weighing shows successive increases in the mass of the sample until it becomes saturated, at which time the weight becomes constant. The time required for equilibrium to be reached varies based on the thickness of the sample and the experimental temperature. This is a very simple technique, requiring little time and equipment. Problems with the gravimetric technique include leaching of materials from the polymer into the donor fluid. Some components that may leach out are impurities, un-reacted hardeners or decomposition products.

The gravimetric absorption technique provides information on material properties for absorbing fluids but it does not provide information on the release of the fluid out of the

polymer. A desorb technique can be used to determine the rate of release for an absorbed fluid. While this technique can give useful results, the permeation of a fluid through a polymer sample cannot be modeled accurately.

Gravimetric techniques are useful to describe material properties; however, in order to accurately predict the loss of a fluid through a membrane, a diffusion cell technique is best. 


\section{Diffusion Test Cells}

There are several methods available for testing samples in a diffusion cell test setup. The first constraint that is imposed on the testing technique is the type of permeant and its expected volume. For the permeability tests in this thesis the permeant was silicone oil. The second constraint is the receiving medium into which the permeant is permeating. In this case, the receiving medium is a fluid, whole bovine blood.

There are many methods for testing the receiving fluid for permeant in the parts per million range (ppm). Some possible methods include gas chromatography with flame ionization detection, infrared spectroscopy, atomic absorption spectroscopy, plasma emission spectroscopy and nuclear magnetic resonance imaging.

Radio labeling the permeant and evaluating its concentration on the receiving side of the diffusion chamber was attempted by Pauley et. al., (1991) and had only limited success due to difficulties with radio labeling impurities in the silicone oil. Inductively coupled plasma atomic emission spectrophotometer testing was used by Matsuo et. al., (1991, 1992) with success. The main problem with this test method is that it determines the number of silicon atoms not silicone oil molecules in the sample. Blood naturaily contains a certain amount of silicon and contamination from glass could easily occur resulting in misleading results. Gas chromatography with mass spectrometry (GC/MS) can quantitatively measure the number of silicone oil molecules, not just silicon atoms, to yield a result indicating the amount of silicone oil in the sample. Easy access to a GC/MS machines through the Carleton University Chemistry Labs encouraged the use of this test method. GC/MS was used for the permeability tests, test evaluations and part of 
the absorption tests. The other part of the absorption tests was performed using the gravimetric technique.

\subsection{Selection of Parameters}

Before a test apparatus and test method can be prepared a selection of test parameters must be made. This was done for each of the test evaluation, absorption and permeability tests so that all relevant parameters would be included in the test procedure.

\section{Chapter 4.2.1 Selection of Parameters - Test Evaluation}

The method used for evaluating silicone oil in the samples from the permeability test was gas chromatography (GC) with mass spectrometry (MS) detection. This test method was chosen because it can characterize and provide quantitative results for low molecular weight silicone oils with excellent accuracy. In order to use the GC/MS, the samples were prepared before injection into the machine since injecting whole blood would ruin the equipment. The method used to prepare the samples was a simple extraction technique where an aggressive solvent is mixed with the blood sample. Most of the silicone oil, lipids, cholesterols, and other hydrophobic compounds dissolved into the solvent. The solvent/blood sample was then centrifuged to remove all of the large blood particles. The clear solvent was then removed and GC/MS tested.

The test evaluations were performed to demonstrate that this method was effective in finding the best solvent for the extraction and determining the partition coefficient of silicone oil between blood and the selected solvent during extraction. The parameters 
that were deemed important for this test were the use of the same donor fluid, receiving fluids and sample volumes used in the permeability tests.

\section{Chapter 4.2.2 Selection of Parameters - Absorption Test}

The silicone oil absorption characteristics of polyurethanes were evaluated by Matsuo et. al., (1991, 1992), to provide some indication of the permeability properties of their elastomers. This test was repeated here in an attempt to accurately obtain the diffusion coefficient of silicone oil into a segmented polyether-polyurethane and to show that silicone oil does in fact absorb into the polymer membrane.

Characteristics that were deemed important for this test were temperature, polymer thickness and donor fluid. A temperature of $37^{\circ} \mathrm{C}$ was used for the absorption tests to model the temperature inside of the human body. The polymer thickness was within the range of 19 to 25 microns or .483 to $.635 \mathrm{~mm}$ as per design specifications for the device.

The donor fluid used for the test was silicone oil. The silicone oil used in the experiment was identical to the fluid used in the EVAD.

The purpose of the absorption test was to obtain diffusion and solubility coefficients through a simple test method so that easy comparisons could be made between candidate diaphragm materials. It should be noted that the absorption tests were not intended to give an indication of the volume of silicone oil that would pass through the diaphragm into blood. This is not possible with this test since no information is obtained on the rate of release of silicone from the diaphragm into a receiving fluid. 


\section{Chapter 4.2.3 Selection of Parameters - Permeability Test}

The first step in modeling a permeation problem is to select the characteristics of the problem that need to be analyzed. The factors that were hypothesized to affect the permeation of silicone oil through the device diaphragm were temperature, diaphragm thickness, diaphragm stress, diaphragm flexing, fluctuating pressure, donor fluid, receiving fluid, boundary conditions and flow over both sides of the diaphragm.

Obviously the ideal test would be to use a prototype of the EVAD. This was not possible since a EVAD was not available and large volumes of blood ( 5 liters) required for mockloop testing is not reasonable. Hence steps were taken to scale down the problem into several small manageable tests where each individual characteristic could be analyzed.

The test apparatus that was used for the permeability test can be seen in Figure 22. The apparatus consisted of an acrylic box with an acrylic lid (see Figure 11,31). Silicone oil was held in the acrylic box while glass tubes (see Figure 18) with Biospan end-caps (see Figure 28) were filled with bovine blood and a small (10 $\mathrm{mm} \mathrm{x} 3 \mathrm{~mm})$ magnetic stir bar. The glass tubes were sealed with PTFE (Teflon) stoppers and suspended on the acrylic lid with the blood filled tubes partially submerged in silicone oil. The level of oil in the acrylic box was adjusted to match the level of the blood in the tubes so that there was not a large hydrostatic pressure gradient across the end-caps.

It was decided that the geometry of the test diaphragms (end-caps) used for the permeability test would be circles with an area $3.14 \mathrm{~cm}^{2}$. This would reduce the volumes of receiving fluid required for the test which was believed to be important since Matsuo 
et. al., $(1991,1992)$ indicated that the full volume of receiving fluid was tested. This addresses concerns over the fact that silicone oil is hydrophobic and may not distribute itself evenly throughout the blood sample. As a result the entire blood sample was tested to determine the total volume of silicone oil permeating through the diaphragm.

The diaphragm samples were bonded, using Biospan, onto the ends of the glass tubes. This reduced the number of locations for silicone oil to contaminate the samples compared to a box type diffusion cell. The only opportunity for silicone oil to enter the tube was to permeate through the diaphragm, through the bond between diaphragm and glass tube or through the top of the glass tube which was located outside of the test apparatus well away from the silicone oil. The diaphragm samples were bonded onto the glass tubes using the same material as the diaphragms (Biospan). The bonding surface was 3 times the size of the current bonding surface used in the prototype EVAD to ensure that the silicone oil would permeate through the diaphragm and not through the bond.

The temperature for the test was selected to be $37^{\circ} \mathrm{C}$ since this is normal body temperature. It should be noted that the actual temperature of the silicone oil in the device when it is implanted and operating is undoubtedly going to be higher than $37^{\circ} \mathrm{C}$ since the silicone oil also works as a coolant. It removes heat from the energy converter and circuits and distributes it to the thoracic cavity tissue through the diaphragms, and the device shell.

The thickness of the diaphragms specified for use in the EVAD range from 19 to 25 microns or .483 to $.635 \mathrm{~mm}$. The diaphragms used in this test all fell within this range. It should be noted that while the diaphragm thickness and material for the permeability test 
are identical to the real diaphragms there is one difference which is the number of casting layers. The diaphragms are solvent cast onto polished aluminum molds. The blood chamber diaphragms require 4 casting layers due to the flow of the polymer over the curved surface. The smaller permeability test diaphragms were cast on much flatter molds and only required 3 casting layers. By reducing the viscosity (by adding the DMAC solvent) of the polymer, it is possible to make the polymer distribute over the mold in such a way that the casting will require 4 layers to meet the required thickness. The change in casting layers from 4 to 3 is not expected to make a significant change in permeability; however, it would be useful to perform a test to evaluate this characteristic.

As mentioned earlier in Section 3.1.2 External Factors Affecting Permeability, fluctuating pressures, stress and flexing on a diaphragm can result in increased or reduced permeability depending on the polymer. It was felt that these characteristics would be difficuit to model accurately and should be analyzed separately. Pressure, stress and flexing of the diaphragms were not analyzed in this test.

The donor fluid used in the permeability tests was decamethyltetrasiloxane. This silicone oil was the same hydraulic fluid that was used in the LVAD. The receiving fluid used was Citrate Phosphate Dextrose Adenine (CPDA) treated bovine blood since no other fluids were found which had the same solubility characteristics as whole blood. Other investigators (CVD/OHI confidential report) have attempted to prepare a solution with similar solubility characteristic to blood but were unable to find a combination that did not swell or dissolve the polymer. A small test was performed to determine the solubility of whole blood and plasma. This test showed that blood is capable of dissolving a considerable amount of silicone oil compared to plasma. Consequently, plasma was 
discarded as a suitable receiving medium since the permeability tests were to investigate the worst case scenario of permeation. An effort was made to find a replacement receiving fluid for blood, since the formed elements in blood breaks down and biological characteristics promote the growth of bacteria, fungi and mold over several days in a test at $37^{\circ} \mathrm{C}$. The tests using whole blood were run up to 18 days at $37^{\circ} \mathrm{C}$; and it should be noted that even after a few days, most of the cells in the blood will be broken down releasing their contents into the solution. This implies that the blood used in the test is not exactly like the blood found in a live patient; however, no other fluid was readily available which had similar dissolving characteristics for silicone oil.

In the EVAD the boundary conditions and flow over the diaphragm on both sides is aggressive during the systolic portion of the pumping cycle. This is due to the forceful ejection of blood from the device that is required to overcome the high back pressure in the aorta. During the diastolic portion of the cycle, the device fills passively and there is a less aggressive flow over the diaphragms. The systolic portion of the cycle lasts for 30 to $35 \%$ of each beat and diastole lasts for the remaining portion of the beat. For the permeability tests, turbulent flow on both sides of the diaphragm was modeled to simulate the worst possible conditions for permeation. This was done by placing a large magnetic stirring bar ( $70 \mathrm{~mm} \times 13 \mathrm{~mm}$ ) in the oil reservoir and small magnetic stirring bar inside of each test tube (10 $\mathrm{mm} \times 3 \mathrm{~mm}$ ). The speed setting on the magnetic stirring tray was 2.5 which resulted in highly turbulent flow eliminating any boundary layer effects.

The magnetic stir bars were also used to stir the blood on the receiving side of the diaphragm. Blood is a heterogeneous solution. If it is left in a static state, the formed 
elements will separate out of the solution into 3 layers, plasma on top, white blood cells (WBC) and platelets in the middle and red blood cells (RBC) on the bottom. It is not acceptable to have only RBC on the surface of the diaphragm because this does not accurately model the receiving fluid. Blood must be continually agitated to ensure that separation does not occur. The use of small magnetic stir bars inside of the test tubes maintained this condition. (Marieb, 1995)

The parameters that were combined into the permeability test included temperature, diaphragm thickness, donor fluid, receiving fluid, boundary conditions and flow over both sides of the diaphragm. The conditions that were not considered in the permeability test included diaphragm stress, diaphragm flexing, and fluctuating pressure.

The purpose of the permeability tests was to prepare a test that would evaluate some of the influencing characteristics that effect the permeation of silicone oil through a segmented polyether-polyurethane diaphragm.

\subsection{Expected Test Results}

Currently, there are no theoretical mathematical models that accurately predict the absorption and diffusion characteristics of silicone oil through polyurethanes into blood. This is because it is very difficult to take into account all of the factors such as swelling, polymer additives, fillers, plasticizers and casting methods that effect the density of the material. Different types of permeants also have independent characteristics which are difficult to characterize. Unlike pure metals which have been well modeled, polymers tend to have characteristics that are individualistic. Since there are many types of 
polymers and modifications to those polymers, they have not all been analyzed. This thesis characterizes the permeability characteristics of a segmented polyetherpolyurethane for permeability and absorption of silicone oil.

\section{Chapter 4.3.1 Expected Test Results - Test Evaluation}

The purpose of the test evaluation was to determine if a given test method was accurate. The two main goals of this test are to discover an effective solvent for extraction of the silicone oil and to determine the partition coefficient of the silicone oil between the solvent and blood. The ideal solvent for the purpose of this extraction is insoluble in water, has high solubility for silicone oil, lower density than plasma (water), forms a manageable solution when mixed with blood, has a high partition coefficient for silicone oil from blood and has a low partition coefficient for blood parts.

It was hoped that a solvent would be found that realized most of the characteristics listed above and had a high partition coefficient for silicone oil near $100 \%$. It is also important to be able to show that the partition coefficient is constant over the range of silicone oil detection that is expected in the permeability tests. Hence when tests are performed with varying concentrations of silicone oil up to $100 \mathrm{ppm}$, the same partition coefficient within standard error should be found for all samples.

The results from the test evaluations will be used as standards to help calculate the volume of silicone oil permeating into the blood samples. 


\section{Chapter 4.3.2 Expected Test Results - Absorption Test}

The absorption tests are expected to show an increase in sample weight as a function of immersion time. Two time dependant regions are expected. The first depicts a rapid rate of uptake of silicone oil over the first short period of time until a partial saturation limit is reached. The second depicts a stable equilibrium amount of silicone oil absorption. This portion of the curve may be at equilibrium, or a very slow rate of oil absorption may continue (see Figure 16).

The first part of the absorption curve provides information on the diffusion coefficient which describes the polymers ability to allow the silicone oil to be absorbed into its volume with no concentration resistance. The second portion of the curve describes the solubility of the silicone oil in the polymer which is the maximum amount of silicone oil that the polymer can absorb.

This is a simple and economical method for determining the material characteristics of the polymer with respect to permeability and diffusion. Using the values obtained here, it will be easy to compare Biospan to other candidate polymers for use as blood diaphragms. This test also indicates whether swelling will be a problem with this polymer by examining the solubility coefficient of silicone oil and water in Biospan. 


\section{Chapter 4.3.3 Expected Test Results - Permeability Test}

The results from the permeability test are expected to show an increasing volume of silicone oil on the receiving side of the diaphragm as a function of immersion time of the tubes in silicone oil. Three time dependant regions are expected. The first region starts with no detection of silicone oil. The second region will show an exponential increase in the detected volume of silicone oil up to the third region which will show a linear increase in the detection of silicone oil with immersion time (see Figure 14).

The first region of the curve where no silicone oil is detected in the sample represents the time it takes for the silicone oil molecules to diffuse into the polymer. The second region of the curve represents the time it takes for the silicone oil to saturate the polymer. As time passes, more and more silicone oil molecules move into the polymer and approach the surface on the receiving side of the diaphragm. The more the molecules approach the receiving side of the diaphragm, the more they permeate into the receiving fluid. This is why the rate of permeation is increasing throughout this region. The third and final region is the portion of the curve where the permeability rate has reached steady state. At this time, the membrane is saturated with silicone oil.

The third section of the curve is expected to remain linear until such time that the concentration of silicone oil in the blood is high enough that the solubility of oil in blood begins to resist the permeation of silicone oil. Eventually the system will reach an equilibrium where there is no net flux of silicone crossing the diaphragm. At this point, the concentration of silicone oil in the samples would be constant. However this is not anticipated since the expected volumes of silicone oil permeating thought the diaphragm 
are small and the solubility limit of silicone oil in blood is high. Also this is a model of an LVAD implanted in a human patient. The silicone oil concentration in circulating human blood is never expected to be high enough to resist permeation since the human body has natural filters (spleen, liver, kidneys, and sweat glands) that are expected to remove silicone molecules from the system. The silicone oil is also lipophilic and is expected to have a tendency to cluster around fat tissue or be evaporated out through the lungs since it has a low vapor pressure.

Another affect that would alter the third portion of the curve from a linear increasing line would be the effects of swelling in the polymer. Some polymers have been know to swell considerably when a permeating species is introduced. In these cases, the swelling process may introduce channels which increase the permeability coefficients of the polymer. The polymer tested here is not known to swell when immersed in silicone oil.

This test will enable the calculation of permeability, diffusion and solubility coefficients specifically for the permeation of silicone oil through Biospan into whole blood. The coefficients can then be used to predict the volume of silicone oil that will be lost through the blood diaphragm of the EVAD over 3 years. 


\section{Chapter 5: Experimental Procedure}

The experimental procedures used in this thesis were developed through several iterations. The procedures listed here are the final versions. The casting of the Biospan end-caps and sheets was performed by a technician at CAE Electronics Ltd. St. Laurent, Quebec, Canada. The manufacturing process of the polymers is proprietary information and while inmportant it is not discussed in this thesis.

\subsection{Experimental Procedure - Test Evaluation}

Test evaluation was performed to demonstrate that silicone oil could be extracted from blood and quantified accurately. This test was also used to determine the best solvent for the permeability tests.

\subsubsection{Sample Preparation}

1) prepared and labeled $40 \mathrm{ml}$ glass scintillation vial(Sharpie permanent marker)

2) injected $3 \mathrm{ml}$ of Citrate Phosphate Dextrose Adenine (CPDA) treated bovine blood into glass scintillation vial (10 $\mathrm{ml}$ syringe) 
3) injected a volume of silicone oil ( $10 \mu \mathrm{l}$ syringe) into scintillation vial on top of blood (desired volume determined by calculating desired ppm of silicone oil in extraction solvent, not ppm in blood)

Example: 100 ppm standard (by volume) for GC testing. Therefore, a solvent sample with $100 \mathrm{ppm}$ of silicone oil is required. The intention is to use $20 \mathrm{ml}$ of dichloromethane (DCM) to extract the silicone oil from the blood. $2 \mu \mathrm{l}$ of silicone oil must be injected into the blood.

Note: This works out to $267 \mathrm{ppm}$ of silicone oil in blood.

4) injected $4.5 \mathrm{ml}$ of Citrate Phosphate Dextrose Adenine (CPDA) treated bovine blood into glass scintillation vial (10 ml syringe)

5) placed PTFE-covered magnetic stir bar ( $8 \mathrm{~mm} \times 3 \mathrm{~mm})$ in glass scintillation vial

6) put Teflon lined cap on glass scintillation vial

7) placed glass scintillation vial on magnetic stir tray

\subsubsection{Removal From Test Setup}

1) mixed sample on stir tray for 2 days

2) removed glass scintillation vial from stir tray

3) transferred all $7.5 \mathrm{ml}$ of blood (Pasteur pipette) from test tube to $40 \mathrm{ml}$ glass scintillation vial

4) labeled glass scintillation vial with sample number (Sharpie permanent marker)

5) added $10 \mathrm{ml}$ of desired solvent to blood in $40 \mathrm{ml}$ glass scintillation vial

6) placed PTFE-lined cap on glass scintillation vial

7) placed bottle on $45^{\circ}$ inclined Chuo-Denki turn table

8) set speed at 45 RPM 
9) extracted sample for 2 days

10) removed sample from turn table and left to sit for 10 minutes

(to allow fluid to sink to bottom of tube before venting)

11) vented sample by opening cap to release pressure

12) screwed top back on

13) placed glass scintillation vial in refrigerated centrifuge

Note: If dichloromethane (DCM) solvent is used the cap must be left slightly unscrewed to relieve pressure buildup. Otherwise, the vial will explode in the centrifuge.

14) lowered centrifuge temperature to $15^{\circ} \mathrm{C}$ to promote complete separation

15) set centrifuge speed at 3000 RPM (1875 G) for 20 minutes

16) removed sample from centrifuge

17) cleaned and labeled $40 \mathrm{ml}$ glass scintillation vial with sample number for pure solvent . (Sharpie permanent marker)

18) transferred solvent from centrifuged sample into pure solvent scintillation vial

19) put PTFE-lined cap on pure solvent scintillation vial

20) repeated steps 5 to 19 two times (total of three $10 \mathrm{ml}$ solvent extractions)

\subsubsection{Preparation for GC testing}

1) assembled $1.5 \mathrm{ml} \mathrm{GC}$ vial and labeled with sample number

2) transferred extraction solvent from pure solvent scintillation vial using Pasteur pipette

3) placed PTFE lined cap on vial

4) stored vial in fridge at $1{ }^{\circ} \mathrm{C}$ until GC testing 


\subsubsection{GC Testing}

GC testing was performed at Carleton University's Centre for Analytical and Environmental Chemistry. A GC method and ACU (acquisition program) method were prepared specifically to detect silicone oil extracted from blood samples. These methods are listed in Appendix D.

Standards that were used for the test evaluation were directly prepared silicone oil standards.

1) loaded Gas Chromatography machine with GC method and ACU method specifically prepared for detection of silicone oil in blood

2) injected pure solvent to ensure GC column was clean

3) cleaned syringe 10 times using clean solvent

4) cleaned syringe 10 times with next sample

5) took $1.8 \mu \mathrm{l}$ of sample into syringe (no bubbles)

6) injected sample into GC/MS machine

7) recorded volume remaining in syringe $(.2-6 \mu \mathrm{l})$

8) cleaned syringe 10 times using clean solvent

9) observed plotted chromatogram and saved to disk

10) recorded silicone oil peak time, height and area

11) calculated concentration of injected sample using standards 


\subsection{Experimental Procedure - Absorption Test}

There were two methods used for the absorption tests, GC testing and gravimetric testing. GC testing extracts the silicone oil from the Biospan samples which have been soaked in silicone oil for different periods of time. Gravimetric testing uses one piece of Biospan at a time and monitors the weight of the piece at progressive time intervals.

There were 2 GC tests and 2 gravimetric tests. The first GC test extracted silicone oil from end-caps that were used in the permeability test. The second GC test used 5 Biospan sheets. Both gravimetric tests used Biospan sheets. Since there were 3 different test methods, 3 different procedures must be listed here: Absorption Procedure 1: GC Test End-Cap, Absorption Procedure 2: GC Test Sheet and Absorption Procedure 3: Gravimetric Tests.

\subsubsection{Absorption Procedure 1: GC Test End-Cap}

As mentioned earlier, the samples used in this test were the end-caps used in the permeability tests.

\subsubsection{Sample Preparation}

1) cleaned Biospan end-cap with $95 \%$ ethyl alcohol to remove surface contaminants

2) dried end-cap in an oven at $50^{\circ} \mathrm{C}$ for 24 hours

3) removed end-cap from oven and weighed it (used scale +-.0001 g)

4) measured thickness using a digital depth gauge

5) placed end-cap on bottom of test tubes (used tweezers and non-latex gloves) 
6) filled $10 \mathrm{cc}$ syringe with fluid Biospan

7) placed blunt tip of Biospan syringe in between the end-cap and the test tube

8) rotated test tube while Biospan was injected

9) inspected end-cap for bubbles and removed any bonding defects

10) returned test tube to oven to dry at $50^{\circ} \mathrm{C}$ for 48 hours

11) removed test tube from oven and rinsed briefly with $95 \%$ ethyl alcohol and returned to oven for 24 hours

12) washed (95\% ethyl alcohol) PTFE-stopper and PTFE-covered magnetic stir bar ( 8 $\mathrm{mm} \times 3 \mathrm{~mm}$ ) and placed in oven for 24 hours

13) removed test tube from oven

14) placed PTFE covered magnetic stir bar $(8 \mathrm{~mm} \times 3 \mathrm{~mm})$ inside of test tube

15) extracted $7.5 \mathrm{ml}$ of bovine blood from sampling port in blood bag using Precision Glide Needle (22 G $\left.1 \frac{1}{2}\right)$ and syringe $(10 \mathrm{cc})$

16) added $7.5 \mathrm{ml}$ of blood to test tube

17) placed PTFE stopper in top of test tube

18) labeled test tube and PTFE stopper sample number (Sharpie permanent marker)

\subsubsection{Test Setup}

1) placed magnetic stir tray in incubator

2) set stir tray speed at 2.5

3) added $.8 \mathrm{~L}$ silicone oil to test apparatus reservoir

4) placed top of test apparatus on reservoir

5) placed PTFE-coated magnetic stir bar $(70 \mathrm{~mm} \times 13 \mathrm{~mm})$ in reservoir 
6) placed modified polypropylene centrifuge tubes in test apparatus holes (to prevent silicone oil evaporation)

7) placed assembled test apparatus in incubator

8) placed thermometer into silicone oil fluid

9) adjusted incubator temperature gauge until silicone oil fluid temperature reached constant $37^{\circ} \mathrm{C}$

The following procedure was repeated for each test sample:

10) removed thermometer and test apparatus from incubator

11) removed modified polypropylene centrifuge tube from test apparatus

12) prepared new tube as per Section 5.2.1.2 Sample Preparation and placed it in empty location in test apparatus

13) retumed test apparatus to incubator

14) centered test apparatus on magnetic stir tray

15) returned thermometer test apparatus

16) monitored temperature to make sure it remained at $37^{\circ} \mathrm{C}$

\subsubsection{Removal From Test Setup}

Removal of each sample from the test setup depended on the desired duration for the test. Test durations varied from 4 hours to 18 days.

1) removed thermometer from test apparatus

2) removed test apparatus from incubator 
3) removed desired tube from apparatus (no silicone oil dripped on other tubes during removal)

4) hung test tube on rack

5) removed PTFE-stopper

๑) removed all $7.5 \mathrm{ml}$ of blood from the test tube (used Pasteur pipette)

7) removed end-cap from test tube (used tweezers)

8) removed silicone oil and blood from surface of end-cap using lint-free tissue

9) put end-cap in $40 \mathrm{ml}$ glass scintillation vial

10) added $15 \mathrm{ml}$ dichloromethane (DCM) to scintillation vial for extraction

11) extracted samples for 2 days

\subsubsection{Preparation For GC Testing}

1) prepared and labeled $1.5 \mathrm{ml}$ glass sample vial (used Sharpie permanent marker)

2) injected pure dichloromethane (DCM) into sample vial $(1.5 \mathrm{ml})$ to dilute sample

3) removed extracted solvent glass scintiliation vial using micro liter syringe

4) injected volume of extracted solvent into sample vial $(1.5 \mathrm{ml})$ to reduce expected concentration below $100 \mathrm{ppm}$

5) placed cap on vial

6) stored vial in fridge at $1{ }^{\circ} \mathrm{C}$ until $\mathrm{GC}$ testing 


\subsubsection{GC Testing}

GC testing was performed at Carleton University's Centre for Analytical and Environmental Chemistry. A GC method and ACU method were prepared specifically to detect silicone oil extracted from blood samples. See Appendix D.

Standards that were used for the absorption end-cap GC tests were prepared by directly injecting a known volume of silicone oil into pure dichloromethane (DCM).

1) loaded Gas Chromatography machine with GC method and ACU method specifically prepared for detection of silicone oil in blood

2) injected pure solvent to ensure GC column was clean

3) cleaned syringe 10 times using clean solvent

4) cleaned syringe 10 times with next sample

5) took $1.8 \mu \mathrm{l}$ of sample into syringe (no bubbles)

6) injected sample into GC/MS machine

7) recorded volume remaining in syringe $(.2-.6 \mu \mathrm{l})$

8) cleaned syringe 10 times using clean solvent

9) observed plotted chromatogram and saved to disk

10) recorded silicone oil peak time, height and area

11) calculated concentration of injected sample using standards 


\subsubsection{Absorption Procedure 2: GC Test Sheet}

Biospan samples used for this test were taken from flat cast sheets used to test casting methods. Test samples were cut from the sheets into pieces measuring 1 in $\times 3$ in $\times .5$ $\mathrm{mm}$.

\subsubsection{Sample Preparation}

1) cleaned 5 Biospan test samples (used $95 \%$ ethyl alcohol)

2) dried test samples in oven at $50^{\circ} \mathrm{C}$ for 24 hours

3) removed samples from oven

4) weighed samples (used scale accurate to +-.0001 g)

5) measured thickness of sample (used digital depth gauge)

\subsubsection{Test Setup}

1) filled 5 glass bottles $(60 \mathrm{ml})$ with $50 \mathrm{ml}$ of silicone oil

2) preheated incubator and silicone oil filled glass bottles to $37^{\circ} \mathrm{C}$

3) placed Biospan test samples in glass bottles

4) screwed on bottle lids

5) returned bottles to incubator at $37^{\circ} \mathrm{C}$ 


\subsubsection{Removal From Test Setup}

All five samples were removed after 2 days so that an accuracy comparison of the results could be made.

1) removed all 5 bottles from the incubator

2) removed adherent silicone oil from each sample (used lint-free tissue)

3) placed dried samples in empty glass bottles $(60 \mathrm{ml})$

4) added extraction solvent (extracted 3 samples with $20 \mathrm{ml}$ hexane)

5) added extraction solvent (extracted remaining 2 samples with $20 \mathrm{ml}$ dichloromethane (DCM))

6) extracted samples for 2 days

\subsubsection{Preparation For GC Testing}

1) prepared glass sample vial $(1.5 \mathrm{ml}$ ) (used Sharpie permanent marker)

2) injected pure solvent into sample vial $(1.5 \mathrm{ml})$ in preparation for dilution of sample note: The same solvent was used for dilution as was used for extraction of the sample.

3) removed extracted solvent from glass bottle using micro liter syringe

4) injected extracted solvent into $1.5 \mathrm{ml}$ sample vial to reduce expected concentration below $100 \mathrm{ppm}$

5) placed PTFE-lined cap on vial

6) stored vial in fridge at $1{ }^{\circ} \mathrm{C}$ until $\mathrm{GC}$ testing 


\subsubsection{GC Testing}

GC testing was performed at Carleton University's Centre for Analytical and Environmental Chemistry. A GC method and ACU method were prepared specifically to detect silicone oil extracted from Biospan samples using either hexane or dichloromethane (DCM) as the extraction solvent. See Appendix D.

Standards that were used for the absorption end-cap GC tests were prepared by directly injecting a known volume of silicone oil into pure dichloromethane (DCM) and hexane.

1) loaded Gas Chromatography machine with GC method and ACU method specifically prepared for detection of silicone oil in blood

2) injected pure solvent to ensure GC column was clean

3) cleaned syringe 10 times using clean solvent

4) cleaned syringe 10 times with next sample

5) took $1.8 \mu$ l of sample into syringe (no bubbles)

6) injected sample into GC/MS machine

7) recorded volume remaining in syringe $(.2-.6 \mu \mathrm{l})$

8) cleaned syringe 10 times using clean solvent

9) observed plotted chromatogram and saved to disk

10) recorded silicone oil peak time, height and area

11) calculated concentration of injected sample using standards 


\subsubsection{Absorption Procedure 3: Gravimetric Tests}

\subsubsection{Sample Preparation}

1) cleaned Biospan test sample (used $95 \%$ ethyl alcohol)

2) dried test sample in oven at $50^{\circ} \mathrm{C}$ for 24 hours

3) removed test sample from oven (weighed using scale $+-.0001 \mathrm{~g}$ )

4) measured thickness of sample (used digital depth gauge)

\subsubsection{Test Setup}

1) filled clear glass bottle $(500 \mathrm{ml})$ with $400 \mathrm{ml}$ of silicone oil

2) preheated incubator and silicone oil filled glass bottle to $37^{\circ} \mathrm{C}$

3) placed wire clips through holes in clear glass bottle $(500 \mathrm{ml})$ cap

4) placed Biospan test sample in clips and screwed top onto bottle

5) returned bottle to incubator at $37^{\circ} \mathrm{C}$

\subsubsection{Removal \& Return To Test Setup}

Removal and return to test setup was repeated at varying time periods. Time periods were sequentially increased so that excessive data was not obtained and a smooth mass increase curve was observed.

1) removed bottle from incubator

2) recorded time of removal 
3) removed sample from bottle

4) removed adherent silicone oil from sample surface (used lint free tissues)

5) weighed sample (used scale +-.0001 g)

6) recorded sample weight

7) returned sample to bottle

8) recorded time of submersion

9) repeated Steps 1 to 8 until no mass increase was detected

10) analyzed and plotted results

\subsection{Experimental Procedure - Permeability Test}

The permeability test procedure listed here is the final version arrived at through several experimental iterations. It was developed by the author.

\subsubsection{Sample Preparation}

1) cleaned Biospan end-cap with $95 \%$ ethyl alcohol to remove surface contaminants

2) dried end-cap in an oven at $50^{\circ} \mathrm{C}$ for 24 hours

3) removed end-cap from oven and weighed it (used scale +-.0001 g)

4) measured thickness using a digital depth gauge

5) placed end-cap on bottom of test tubes (used tweezers and non-latex gloves)

6) filled $10 \mathrm{cc}$ syringe with fluid Biospan

7) placed blunt tip of Biospan syringe in between the end-cap and the glass test tube

8) rotated test tube while Biospan was injected

9) inspected end-cap for bubbles and removed any bonding defects 
10) returned test tube to oven to dry at $50^{\circ} \mathrm{C}$ for 48 hours

11) removed test tube from oven and rinsed briefly with $95 \%$ ethyl alcohol and returned to oven for 24 hours

12) washed ( $95 \%$ ethyl alcohol) PTFE-stopper and PTFE-covered magnetic stir bar ( 8 $\mathrm{mm} \times 3 \mathrm{~mm}$ ) and placed in oven for 24 hours

13) removed test tube from oven

14) placed PTFE covered magnetic stir bar $(8 \mathrm{~mm} \times 3 \mathrm{~mm})$ inside of test tube

15) extracted $7.5 \mathrm{ml}$ of bovine blood from sampling port in blood bag using Precision

Glide Needle (22 G $\left.1 \frac{1}{2}\right)$ and syringe $(10 \mathrm{cc})$

16) added $7.5 \mathrm{ml}$ of blood to test tube

17) placed PTFE stopper in top of test tube

18) labeled test tube and PTFE stopper sample number (Sharpie permanent marker)

\subsubsection{Test Setup}

1) placed magnetic stir tray in incubator

2) set stir tray speed at 2.5

3) added $.8 \mathrm{~L}$ silicone oil to test apparatus reservoir

4) placed top of test apparatus on reservoir

5) placed PTFE-coated magnetic stir bar (70 $\mathrm{mm} \times 13 \mathrm{~mm})$ in reservoir

6) placed modified polypropylene centrifuge tubes in test apparatus holes (to prevent silicone oil evaporation)

7) placed assembled test apparatus in incubator

8) placed thermometer into silicone oil fluid 
9) adjusted incubator temperature gauge until silicone oil fluid temperature reached constant $37^{\circ} \mathrm{C}$

The following procedure was repeated for each test sample:

10) removed thermometer and test apparatus from incubator

11) removed modified polypropylene centrifuge tube from test apparatus

12) prepared new tube as per Section 5.3.1 Sample Preparation and placed it in empty location in test apparatus

13) returned test apparatus to incubator

14) centered test apparatus on magnetic stir tray

15) returned thermometer test apparatus

16) monitored temperature to make sure it remained at $37^{\circ} \mathrm{C}$

\subsubsection{Removal from Test Setup}

Removal of each sample from the test setup depended on the desired duration for the test. Test durations varied from 4 hours to 18 days.

1) removed thermometer from test apparatus

2) removed test apparatus from incubator

3) removed desired tube from test apparatus (no silicone oil dripped on other tubes)

4) hung test tube on rack

5) removed PTFE-stopper 
6) transferred all $7.5 \mathrm{ml}$ of blood from test tube to glass scintillation vial (40 $\mathrm{ml}$ ) (used Pasteur pipette)

7) labeled glass scintillation vial with sample number

8) added $10 \mathrm{ml}$ of desired solvent to blood in $\mathbf{4 0 ~} \mathrm{ml}$ glass scintillation vial

9) placed PTFE-lined cap on glass scintillation vial

10) placed bottle on $45^{\circ}$ inclined Chuo-Denki turn table

11) set speed at 45 RPM

12) extracted sample for 2 days

13) removed sample from turn table and left to sit for 10 minutes

(to allow fluid to sink to bottom of tube before venting)

14) vented sample by opening cap to release pressure

15) screwed top back on

16) placed glass scintillation vial in refrigerated centrifuge

Note: If DCM solvent is used the cap must be left slightly unscrewed to relieve pressure buildup. Otherwise, the vial will explode in the centrifuge.

17) lowered centrifuge temperature to $15^{\circ} \mathrm{C}$ to promote complete separation

18) set centrifuge speed at $3000 \mathrm{RPM}(1875 \mathrm{G})$ for 20 minutes

19) removed sample from centrifuge

20) cleaned and labeled $40 \mathrm{ml}$ glass scintillation vial with sample number for pure solvent (Sharpie permanent marker)

21) transferred solvent from centrifuged sample into pure solvent scintillation vial

22) put PTFE-lined cap on pure solvent scintillation vial

23) repeat steps 8 to 22

24) repeat steps 8 to 22 again (total of three $10 \mathrm{ml}$ soivent extractions) 


\subsubsection{Preparation for GC Testing}

1) assembled $1.5 \mathrm{ml} \mathrm{GC}$ vial and labeled with sample number

2) transferred extraction solvent from pure solvent scintillation vial using Pasteur pipette

3) placed PTFE lined cap on vial

4) stored vial in fridge at $1{ }^{\circ} \mathrm{C}$ until GC testing

\subsubsection{GC Testing}

GC testing was performed at Carleton University's Centre for Analytical and Environmental Chemistry. A GC method and ACU method were prepared specifically to detect silicone oil extracted from blood samples. See Appendix D.

Standards that were used for the permeability GC tests were extraction samples from the test evaluations. Directly prepared silicone oil standards were also used at every GC test to ensure that there were no detection errors.

1) loaded Gas Chromatography machine with GC method and ACU method specifically prepared for detection of silicone oil in blood

2) injected pure solvent to ensure GC column was clean

3) cleaned syringe 10 times using clean solvent

4) cleaned syringe 10 times with next sample

5) took $1.8 \mu$ l of sample into syringe (no bubbles)

6) injected sample into GC/MS machine

7) recorded volume remaining in syringe $(.2-.6 \mu l)$ 
8) cleaned syringe 10 times using clean solvent

9) observed plotted chromatogram and saved to disk

10) recorded silicone oil peak time, height and area

11) calculated concentration of injected sample using standards 


\section{Chapter 6: Results and Calculations}

The following sections analyze the data that was accumulated while performing the Test Evaluation, the Absorption Tests and the Permeability Tests.

\subsection{Observations and Results - Test Evaluation}

The Test Evaluations were performed to demonstrate that the extraction methods used to remove the silicone oil from the blood samples (in the permeability test) would be effective. This test directly injected a known volume of silicone oil into a volume of blood (same volume as used for the permeability tests). The same extraction procedure was performed on the test evaluation samples as was performed on the permeability test samples.

\subsubsection{Data Acquisition and Quantitative Results - Test Evaluation}

The technique used to acquire the samples is listed in Chapter 5.1 Experimental Procedure - Test Evaluation. Samples of the graphs produced by the GC/MS equipment are presented in Appendix D. These graphs (see Figure 46) show detection peaks at times 
with respect to the maximum peak area. Using the MS, the composition of the compound detected at a given peak can be analyzed. When 295 (see Figure 48) is seen on the MS graph, silicone oil is identified. A second smaller peak can be seen at 348. The MS indicates 355 as a major component of the peak. The compound detected is most likely a contaminant in the silicone oil.

The volume of silicone oil detected in a sample is calculated using standards and the measured peak size from the GC. For the test evaluations, the standards are samples of the extraction solvent into which a known volume of silicone oil has been directly injected. When the sample was GC tested these standards yielded a peak which was used as a reference to determine if the test evaluation samples peaks represent full or partial extraction of the silicone oil from blood. The following equation was used to determine the standard coefficient (Coef snd $_{\text {sin }}$ :

$$
\operatorname{Coef}_{\text {strd }}=\frac{A R E A_{G C}}{V_{\text {trij }} * C_{e x t}}
$$

where $A R E A_{G C}$ is the area of the silicone oil peak detected by the GC, $V_{\text {inj }}$ is the volume of the sample that was injected into the $G C$ injector and $C_{e x t}$ is the known concentration of silicone oil in the volume of solvent used for extraction (ppm). The volume of silicone oil in the test evaluation sample was calculated from the area determined using the GC program. Once the peak had been identified as silicone oil, the area was used in the following calculation to determine the concentration of silicone oil in the volume of extraction solvent:

$$
C_{\text {ext }}=\frac{A R E A_{G C}}{V_{\text {iny }} * \operatorname{Coef}_{\text {stind }}}
$$


where $\mathrm{AREA}_{G C}$ is the area of the silicone oil peak detected by the $G C$ for the permeability sample, $V_{\text {inj }}$ is the volume of the sample that was injected into the $G C$ injector and Coef is the coefficient standard calculated using Equation 17.

Once the concentration of the sample is calculated (in ppm), the volume of silicone oil in the extraction solvent can be calculated using the following equation:

$$
V_{S I}=\frac{V_{e x t} * C_{e x t}}{1,000,000}
$$

where $\mathrm{V}_{\mathrm{SI}}$ is the volume of silicone oil in the total volume of extraction solvent, $\mathrm{V}_{\mathrm{ext}}$ is the total volume of extraction solvent, and $\mathrm{C}_{\mathrm{ext}}$ is the concentration of the extraction solvent calculated using Equation 18.

The volume of silicone oil that is in the extraction solvent can then be compared to the known volume of the silicone oil that was injected into the blood sample. If the volume of silicone oil that is extracted from the sample is less than the volume injected, there is a partitioning of silicone oil between the blood and the solvent. Care was taken to ensure that this did not affect the test results.

These calculations were performed on the samples taken in the test evaluation. The results of the test evaluation can be seen in Table 4.

From these results, it can be seen that the extraction procedure provides results that are close to the expected values. It appears that the solvent extracts more silicone oil than is actually in the sample. This was likely caused by errors in injecting a small volumie of 
silicone oil into the standard, or the blood. Also standard error in GC testing also contributes to the variability in the results.

\subsubsection{Observations and Qualitative Results - Test Evaluation}

The process used to mix the samples was not as aggressive or as intensive as the mixing of the samples in the permeability test using magnetic stir bars. Furthermore, the samples were not mixed at elevated temperatures. As a result the RBC's were not lysed and the samples did not turn dark brown.

After centrifuging the samples in the extraction procedure, the samples yielded a white layer between the red layer and the hexane solvent which was not seen in the permeability test results.

Centrifuging the samples did not remove all of the solvent from the blood. It was found that approximately $5 \mathrm{ml}$ out of $30 \mathrm{ml}$ of the solvent remained in the blood after centrifuging. This may have contributed to the higher than expected experimental standard results (see Table 4). Higher centrifuge speeds could be used to attempt to fully separate the samples.

\subsection{Results - Absorption Test}

The absorption tests were performed to isolate and evaluate the material characteristics of Biospan with respect to permeability and diffusion. A GC end-cap test, a GC sheet test 
and two gravimetric tests were performed. Each of the 3 tests were performed to evaluate different material properties.

\subsubsection{Data Acquisition and Quantitative Results - Absorption Test}

\section{GC Method}

The end-cap and sheet absorption tests using the GC method had similar data reduction steps and will be discussed together. The technique used to acquire the samples is listed in Sections 5.2.1 and 5.2.2. Samples of the graphs produced by the GC/MS equipment are presented in Appendix D. These graphs show detection peaks of compound suspended in the sample solvent. The compounds are separated using a column so that each component is analyzed at a different time. The size (integrated area) of the peak represents the volume of the compound that is detected. Using the MS, the composition of the compound detected at a given peak can be analyzed. When 295 is seen on the mass spectrum, silicone oil is identified.

The volume of silicone oil detected in a sample is calculated using standards. Standards for the absorption test are pure solvent samples that have had silicone oil directly injected into their known volume. This implies that the results from the GC/MS will indicate specifically the volume of silicone oil in the extraction solvent. If there is partitioning of silicone oil between the solvent and the polymer, some of the silicone oil will remain in the polymer and all of it will not be detected. A comparison between the gravimetric absorption tests and the GC absorption tests will provide some information on the partitioning characteristics of the polymer and the solvents used in this test. 
Testing the standards generates a silicone oil peak. The area of the peak is calculated by the GC program and displayed on the graph as the third number in the column (see Figure 46). (The first number is the retention time and the second number is the peak height.) The area is then used to calculate the standard coefficient $\left(\right.$ Coef $\left._{\text {stad }}\right)$.

$$
\operatorname{Coef}_{\text {strd }}=\frac{A R E A_{G C}}{V_{\text {inj }} * C_{\text {snd }}}
$$

where AREA $_{G C}$ is the area of the silicone oil peak detected by the GC, $V_{\text {ij }}$ is the volume of the sample that was injected into the $G C$ injector and $C_{\text {smd }}$ is the known concentration of silicone oil directly injected into the solvent.

The vclume of silicone oil in the diluted absorption test sample was calculated from the peak area which was measured using the GC. Once the peak has been identified as silicone oil, the area is used in the following calculation to determine the concentration of silicone oil in the diluted volume of extraction solvent $\left(\mathrm{C}_{\text {dilex }}\right)$.

$$
C_{\text {dilext }}=\frac{A R E A_{G C}}{V_{\text {trnj }} * \text { Coef }_{\text {stond }}}
$$

where $\mathrm{AREA}_{\mathrm{GC}}$ is the area of the silicone oil peak detected by the GC for the permeability sample, $V_{i n j}$ is the volume of the sample that was injected into the $G C$ injector and Coef stnd $_{\text {ind }}$ is the coefficient standard calculated using Equation 17.

Using the concentration of silicone oil in the dilution sample $\left(\mathrm{C}_{\text {dilex }}\right)$, we can find the concentration of silicone oil in the extraction volume $\left(\mathrm{C}_{\mathrm{ex}}\right)$. 


$$
C_{\text {ext }}=\frac{\left(V_{\text {diled }}+V_{\text {dilm }}\right) * C_{\text {dilext }}}{V_{\text {diled }}}
$$

where $V_{\text {diled }}$ is the diluted volume and $V_{\text {dilm }}$ is the dilution volume of pure solvent. $C_{\text {dilext }}$ is the concentration of silicone oil in the diluted sample obtained from Equation 20.

Once the concentration of the diluted sample is calculated, the concentration of the sample before dilution can be found:

$$
V_{S I}=\frac{V_{e x t} * C_{e x t}}{1,000,000}
$$

The volume of silicone oil that was in the extraction solvent was the total volume of silicone oil that had been absorbed into the Biospan samples. Figures 32 and 33 were prepared to show the results of the GC tests.

\section{GC End-Cap Test}

Figure 32 and Table 5 show the mass of silicone oil absorbed by the polymer end-caps and the ratio of absorbed mass to initial mass of the end-cap. Figure 32 is presented in terms of solubility mass/mass (g/g) vs. time (days). Examination of the results indicates nearly steady state conditions with the possibility of a slightly decreasing trend. 
The expected results for this test is a curve with two time dependant regions. The first depicts a rapid rate of uptake of silicone oil over the first short period of time until a partial saturation limit is reached. The second depicts a stable equilibrium amount of silicone oil absorption. Figure 32 does not show the expected first region of the graph where there is a rapid uptake of silicone oil. This was due to the first sampling point occurring after saturation occurred. The second region was seen where the sample had reached steady state; however, the apparent decreasing trend in silicone oil absorption was not expected and is difficult to explain.

The mean solubility of the end-caps was calculated and found to be $.0497 \mathrm{~g} / \mathrm{g}$. The curve does not show the absorption of silicone oil for the time up to steady state; and therefore, no calculations for the diffusion coefficient were made.

\section{GC Sheet Test}

The GC sheet test was performed to determine the reliability and accuracy of the absorption tests using GC testing with 2 candidate extraction solvents, Dichloromethane (DCM) and hexane. The test procedure can be seen in Section 5.2.2. The results from this test are shown in Figure 33 and Table 6. Figure 33 is presented in terms of solubility mass/mass ( $g / g)$ vs. sample number since all samples were taken at 2 days. Examination of the results indicates that there was a large variation in extraction volumes of silicone oil between samples that were extracted with DCM and hexane. The results indicate good consistency between the samples tested using the same solvents. The apparent solubility of the three hexane-extracted samples were $.0310, .0381$ and .0382 . The apparent solubility of the two DCM-extracted samples were .0511 and .0519 . This shows 
that the GC test method is precise and repeatable. The samples used for the hexane and DCM extractions were identical and therefore absorbed the same volumes of silicone oil but this was not seen in the experimental results (see Figure 33). Clearly hexane is not as effective a solvent for extracting silicone oil from Biospan as DCM. The fact that hexane extracted less silicone oil than DCM indicates that there is a larger partition coefficient between the polymer and the hexane solvent than there is between the polymer and the DCM.

This test made no attempt to determine the curve describing the rapid uptake of silicone oil into the Biospan samples; hence no attempts were made to determine the diffusion coefficient.

\section{Gravimetric Test}

The gravimetric test was performed to determine the absorption performance of the material during the rapid uptake of silicone oil before it reached steady state and to verify the test results from the GC absorption tests. The method used to acquire the test sample results is described in Section 5.2.3.

The results from the gravimetric tests were plotted in terms of mass (g) or apparent solubility (g/g) vs. time (s). Acquiring and analyzing the data was very simple in this case with the solubility coefficient being the only calculation:

$$
S_{t}=\frac{M_{t}-M_{t=0}}{M_{t=0}}
$$


where $S_{t}$ is the solubility at a time $(t), M_{t}$ is the mass of the sample at time $(t)$ and $M_{t=0}$ is the initial mass of the sample before it is immersed in the silicone oil bath. The solubility of the material is taken at the time where the sample absorbed the maximum volume of silicone oil (see Figure 34 and 35 and Table 7 and 8).

The resulting curve was as expected with two distinct regions. The first region depicting a rapid uptake of silicone oil was clearly seen to occur during the first 24 hours of both gravimetric tests. The second region where the silicone oil uptake has reached steady state can be seen from 24 hours after the test started until the test is terminated.

The two gravimetric tests indicated that the solubility coefficient of a $.65 \mathrm{~mm}$ thick sheet is .052 and the solubility coefficient of a $.60 \mathrm{~mm}$ thick sheet is .038 . The significant change in solubility between two samples of different thickness may indicate that the ability of the Biospan polyurethane to absorb silicone oil is highly dependant on the thickness of the polymer. However, other variables influence the value such as the free volume in the polymer which is affected by the manufacturing of the material and its casting method.

Since this test method provides accurate information on the rapid uptake of silicone oil into the polymer matrix, a calculation can be made to determine the diffusion coefficient of the polymer:

$$
\frac{M_{t}}{M_{f}}=4\left(\frac{D t}{\pi l^{2}}\right)^{1 / 2}
$$

This equation can be rearranged to solve for the diffusion coefficient (D). 


$$
D=\left(\frac{M / M_{f}}{t^{1 / 2}}\right)^{2}\left(\frac{l^{2} \pi}{4^{2}}\right)
$$

Using Figure 36 and 37, we can find the slope of the graph.

$$
\text { slope }=\left(\frac{M_{l} / M_{f}}{t^{1 / 2}}\right)
$$

The following equation was used to determine the diffusion coefficient of each test sample.

$$
D=(\text { slope })^{2}\left(\frac{l^{2} \pi}{4^{2}}\right)
$$

where $D$ is the diffusion coefficient, $l$ is the thickness of the sample, $t$ is the time, $M_{t}$ is the mass of silicone oil absorbed by the sample at time $(t)$, and $M_{f}$ is the mass of the silicone oil absorbed once constant mass of the sample had been achieved.

Two Biospan samples were tested using this method, 'B_065' and 'B_060'. Using Equations 22, 25 and 7, the diffusion, solubility and permeability coefficients of the two samples were determined. See table 9.

From these results, we can see similar diffusion coefficients; however, the solubility coefficients vary significantly. The samples used for these tests were not cast on the same sheet and little information is known about the casting process for these samples. 


\subsubsection{Observations and Qualitative Results - Absorption Test}

\section{GC End-Cap Test}

A major source for error in the GC end-cap absorption test is the geometry of the samples. The ideal sample for an absorption test is a flat sheet with a highly uniform thickness across the sample. When samples are to be compared, the samples should be cut from the same sheet and have identical geometries and thicknesses. The end-cap absorption test samples were cast independently of one another. Variations in the permeating surface thickness can be clearly seen in Table 5. The end-caps are not flat (see Figure 28) samples but have vertical surfaces for bonding onto the glass test tubes. All of these factors contribute to the errors in the GC sample technique. One other major source of error is the fact that the Biospan used to bond the end-caps onto the tubes also absorbs silicone oil.

\section{GC Sheet Test}

The GC sheet test showed that the GC test method is reliable since it is repeatable and produced the same extraction values for the samples extracted with the same solvent. This test showed that hexane was not a suitable solvent for extraction since it did not completely extract the silicone oil from the Biospan sample. DCM was the better solvent since it extracted more silicone oil than the Biospan sample. No visible changes were noted in the samples before, during and after testing. 


\section{Gravimetric Test}

The absorption test results were very consistent and the curve was very smooth. Very little variability can be seen in the results and the mass of the samples increased consistently with immersion duration.

Before immersion, the samples were slightly more transparent than after saturation had occurred.

Sources for error in acquiring data for this experiment would have developed from problems with removing the test samples from the incubator. For a certain period of time between each test, both the silicone oil and sample had the opportunity for their temperature to fall below the desired $37^{\circ} \mathrm{C}$. The fact that the samples are removed from the silicone oil and dried using lint-free tissue also disturbs the absorption process as some of the silicone oil absorbed into the sample has the opportunity to evaporate into the atmosphere which reduces the apparent absorption rate. The accuracy of the time interval between taking each sample had some variability; however, a serious attempt was made to minimize the time where the sample was not fully immersed in silicone oil.

\section{Combined Results of Gravimetric and Absorption Tests}

The volume of silicone oil that was in the extraction solvent was the total volume of silicone oil that had been absorbed into the Biospan sample. To show that the samples from the GC tests were similar to the samples from the gravimetric test, a graph was 
prepared (see Figure 38) which combined the data between the end-cap absorption tests and the gravimetric sheet tests.

From this graph, it can be seen that the gravimetric absorption test samples have comparable silicone oil uptake to the GC test extraction samples. Since the extraction technique was proven with the sheet test, the only explanation for the variability in the results for the end-cap samples is variability in the casting method, materials and technique.

\subsection{Observations and Results - Permeability Test}

The qualitative results from the permeability test are presented in Table 10 and graphical representation of the results are shown in Figure 39.

\subsubsection{Data Acquisition and Quantitative Results - Permeability Test}

The technique used to acquire the samples is listed in Section 5.1 Experimental Procedure. Samples of the graphs produced by the GC/MS equipment are presented in Appendix D (see Figures 46, 47, 48 and 49).

The volume of silicone oil detected in a sample is calculated using standards. Standards are samples that are analyzed using the same extraction and processing method as the permeability tests, except a known volume of silicone oil is prepared in the sample. Since the standard is processed using the same method as the permeability test, the percentage of silicone oil extracted from the sample (partition coefficient) is the same. 
The method and calculations used to determine the volume of silicone oil in the permeability test samples is identical to the method and calculations in section 6.1.1 Data Acquisition and Quantitative Results - Test Evaluation and will not be repeated here. The method listed in section 6.1.1 was repeated for each permeability test sample to determine the volume of silicone oil passing through the Biospan end-caps. The volume of silicone oil that was calculated was the total volume of silicone oil that had permeated through the membrane into blood. This is known because the full volume of blood was tested. To determine the permeability and diffusion coefficients, a graph was prepared of all of the permeability test samples. The graph shows a slope that is used to determine the time constant and permeability coefficient of the material.

The graph that shows the results of the permeability test was prepared in terms of the volume of silicone oil passing through the diaphragm per unit area $\left(\mathrm{ml} / \mathrm{cm}^{2}\right)$ vs. time (s). The curves presented in Figures 39 and 40 appeared to follow the trends typical of permeability theory. Three independent regions were visible, however the values of the data in the first two regions are within the standard error of the experiment. The first region where no silicone oil is detected ranges from 0 to 28,000 seconds ( 8 hours). The range from 28,000 seconds ( 8 hours) to 86,000 seconds ( 24 hours) an exponentially increasing volume of detected silicone oil can be seen. The range from 86,000 seconds (24 hours) to the final test point, $1,550,000$ seconds (18 days), the data demonstrated a nearly linear relationship. Close examination of the time from day 8 to day 18 indicated that there may actually be a trend showing a decreasing permeation rate.

These results support the theory presented in Section 4.3 .3 for the first two independent regions of the curve. The third section of the curve may appear linear but actually has a 
decreasing slope. This may be an indication of the silicone oil in the blood samples approaching a saturation limit where the permeation rate of silicone oil through the diaphragm is meeting increasing resistance.

Swelling was not observed to be a problem in these tests. Random sampling of the thickness of the end-caps before and after the permeability tests showed that no significant increase or decrease in end-cap thickness could be observed. The consistently linear portion of the curve indicates that there is not sufficient swelling of the polymer to introduce channels which permit increased diffusion of silicone oil through the end-cap.

The linear portion of the curve is used to determine the permeability and diffusion coefficients. The method of least squares (Miller et. al., 1985) was used to find the most accurate linear approximation to the permeability test data. Only 11 data points from day 1 to day 6 were used in the linear approximation. The data after day 6 indicated a decreasing permeation rate which may have been due to an increasing concentration of silicone oil on the receiving side of the end-caps which would resist permeation. Data points after day 6 were not used because they would decrease the apparent maximum permeation rate leading to incorrect long term predictions. The approximation using the method of least squares for the linear portion of the curve resulted in the following equation:

$$
Y=6.9 * 10^{-10} X-1.3 * 10^{-5}
$$

To find the diffusion coefficient the time constant $(\tau)$ must first be found. The time constant can be found by setting $Y=0$ and solving for $X$ in Equation 26 which results in 
$X=\tau=19253$ seconds. The time constant and thickness can be used to calculate the diffusion coefficient (D).

$$
D=\frac{l^{2}}{6 * \tau}
$$

The thickness $(I)$ of the permeability test end-caps averaged $.0596 \mathrm{~cm}$. This resulted in a diffusion coefficient for the permeability test of $3.1 \times 10^{-8} \mathrm{~cm}^{2} / \mathrm{s}$. The permeability coefficient was calculated using Equation 12. In order to find the permeability coefficient, a derivation was made starting with Equation 12.

$$
Q=\frac{P A t\left(C_{d}\right)}{l}
$$

Where $C_{d}$ is the concentration of the permeant in the donor chamber, $l$ is the thickness of the film and $\mathrm{t}$ is time. The volume of silicone oil permeating thought the diaphragm during the test is negligible compared to the volume of the sample. For the permeability test, the concentration $\left(C_{d}\right)$ of silicone oil in the donor chamber is $100 \%$ or $1.0 \mathrm{~mL} / \mathrm{ml}$. Equation 12 can be rearranged to solve for $P$.

$$
P=\frac{Q l}{A t C_{d}}
$$

The slope determined from the linear portion of the permeability curve is equal to:

$$
\text { slope }=\frac{Q}{A t}
$$

Substituting Equation 28 into Equation 27 we have: 


$$
P=\frac{\text { slope } * l}{C_{d}}
$$

Using Equation 29 the permeability coefficient was determined to be $P=4.1 \times 10^{-11} \mathrm{~cm}^{2} / \mathrm{s}$. The solubility coefficient (S) of the permeability test system was calculated using Equation 7:

$$
S=\frac{P}{D}
$$

The solubility coefficient of the system (S) was determined to be $.00133 \mathrm{~g} / \mathrm{g}$.

Once the coefficients that described the system were identified, an equation was prepared which predicts the permeability characteristics of the EVAD system. It is important to note that the permeability test reported here does not investigate all of the factors that influence the permeability of a polymer diaphragm. Equation 12 was used to predict the volume of silicone oil permeating through the EVAD blood chamber diaphragm using the constants obtained with the permeability test model.

The permeability coefficient (P) was determined from experiments. The area (A) of the blood chamber diaphragm is approximately $60.5 \mathrm{~cm}^{2}$ and the thickness $(l)$ is taken as .060 $\mathrm{cm}$. The concentration of silicone oil in the device $\left(\mathrm{C}_{\mathrm{d}}\right)$ is $100 \%$ or $1.0 \mathrm{ml} / \mathrm{ml}$. The volume permeating at several meaningful durations were calculated at different times $(\mathrm{t})$. See Table 12 for permeation volume predictions for silicone oil loss through the blood chamber diaphragm. 


\subsubsection{Observations and Qualitative Results - Permeability Test}

Samples were initially crimson red and progressively darkened to dark brown over a period of 5 days after permeability testing had started. After 5 days, little change in the colour of the samples was noted (see Figure 41).

No blood clots formed in test tubes where magnetic stir bars were spinning. Early tests did not use magnetic stir bars and resulted in separation of the blood into RBC and plasma layers. There were also large clots located on the sides of the glass tubes where the diaphragm met the glass. The results from these tests indicated a drastically reduced permeation rate which may indicate a strong influence from the stiring motion. A study should be performed on the effects of altering the stiming rate of the samples.

Evaporation was observed inside the sealed test tubes as a fluid was observed condensing in large droplets on the sides of the glass test tubes and the surfaces of the PTFE caps.

Using an acrylic box as a reservoir created some problems in that the magnetic stirring bar spinning on the bottom of the box created acrylic wear particles which could be seen floating throughout the silicone oil reservoir. Figure 25 shows the wear markings caused by the stir bar on the bottom of the reservoir. This resulted in frequent filtering and degassing of the silicone oil in an attempt to keep the contaminants to a minimum.

Immediately after new samples were placed in the test apparatus, the silicone oil beneath the test tube appeared to have a descending flow away from the Biospan diaphragm. It is hypothesized that this effect is a consequence of a cold tube being introduced to warm 
silicone oil. Before injection into the tube, the blood was stored in a fridge at $4^{\circ} \mathrm{C}$. The test apparatus remains at $37^{\circ} \mathrm{C}$ throughout the test. Cold fluids (around the blood-filled testing tube) are more dense than warm fluids (silicone oil in the test apparatus) which results in a convection of cold fluid sinking and warm fluid rising.

Over long duration tests, the silicone oil in the test reservoir developed a yellowing colour. It is known that exposure of the oil to light results in yellowing of the fluid; however, the silicone oil used in this test had minimal exposure. An attempt was made to test a sample of the silicone oil in the reservoir using GC/MS in hopes of finding contaminants. The results were inconclusive, since no other compounds were detected in the sample.

During the experiment the test tubes appeared to developed a vacuum. This could be observed as the Biospan diaphragms located on the bottom of the test tubes were drawn up slightly into the tubes despite the balanced hydrostatic pressures of the fluid inside and outside of the tubes. Another sign of the vacuum in the tubes was the suction sound as the PTFE caps were removed at the end of the tests.

Bonding of the Biospan end-caps to the glass surface was effective. This was determined through random testing of the tubes. Each tube was pressurized and the ends were submerged in water. No bubbles were observed. Visual inspections were made of all end-caps before and after testing. No problems were encountered with bonding. 


\section{Chapter 7: Discussion of Results}

This chapter critically analyzes the test procedures and results for the test evaluation, absorption test and the permeability test.

\subsection{Discussion of Results - Test Evaluation}

The results from the test evaluation verified that the use of gas chromatography is an effective method for quantitatively measuring the volume of silicone oil in a sample of blood. This verifies the test method. The results of the test method were used as standards to determine the volume of silicone oil found in the permeability test samples. The method presented in this thesis shows that sensitive, quantitative monitoring of a low molecular weight polysiloxane in blood is possible. The use of the mass spectrum ensures that the detected peak is in fact decamethyltetrasiloxane.

The key to obtaining complete extraction of silicone oil from the blood sample was to perform 3 separate extractions using the selected solvent, hexane. During the optimization of the extraction procedure, it was found that the first extraction of the blood samples only removed around $70 \%$ of the silicone oil in the sample. 
Extended periods of aggressive mixing on the turn table are required. If aggressive mixing is not performed, the blood remains clustered together and the solvent does not mix uniformly throughout the solution (see Figure 44). Simple observation of the hexane/blood solution can determine if the mixing is complete. A completely mixed solution will appear to be a uniform fluid throughout the volume after sitting for 1 minute. If the mixing process was not aggressive, the solution will separate out with the clear hexane above the dark red blood within 1 minute. Testing of incompletely mixed solutions showed that hexane solvent did not effectively extract the silicone oil. As a result, it is very important to ensure that the samples are properly extracted.

After mixing, a centrifuge was used to separate the dense blood parts from the solvent (see Figure 43). The solvent was then transferred to a "solvent only" vial (see Figure 19). Experience showed that it was not possible to remove $100 \%$ of the solvent from the blood. Of the $30 \mathrm{ml}$ of solvent used for extraction 4 to $5 \mathrm{ml}$ of solvent were not removed from the blood sample and transferred to the "solvent only" vial. This likely contributed to the detected volume of silicone oil in the sample being higher than the actual volume of silicone oil (see Table 4).

One part of the test evaluation was to determine the effectiveness of different solvents for extraction of silicone oil from blood. The two solvents hexane and dichloromethane (DCM) were considered. Hexane was found to be the superior solvent for two reasons. Firstly, the consistency of hexane and blood mixed together produced a slightly viscous fluid which was easy to handle. DCM when mixed with blood had a thick mud-like viscosity that was difficult to handle and stuck to glass surfaces (see Figures $42 \& 44$ ). These figures show blood samples mixed with hexane. Figure 44 shows the samples 
right side up and Figure 42 shows the samples inverted. Examination of the DCM sample shows that the sample is so thick that it sticks to the top of the vial. The higher quality of the hexane sample after centrifuging was the second reason why hexane was selected as the better solvent. When hexane is centrifuged, the RBCs go to the bottom of the tube, leaving a clear yellow solvent sample on top which is easy to transfer out of the vial (see Figure 43). When DCM is centrifuged, the solvent does not separate out of the blood very well and the solvent goes to the bottom of the tube due to its higher density (see Figure 45). DCM samples typically have particles floating in them since the RBC layer must be pierced to remove the solvent sample.

The errors in the test evaluation were limited to standard GC detection errors and errors in preparing the volumes of the samples.

\subsection{Discussion of Results - Absorption Test}

The absorption test was performed to verify that a segmented polyether-polyurethane absorbs silicone oil and to determine the diffusion coefficient of the Biospan material. Two test methods were used, a gravimetric test and a GC test. The results of the two tests yielded similar results; however, only the gravimetric test yielded useful information.

The gravimetric test monitored the silicone oil uptake very accurately providing a smooth curve of silicone oil uptake with respect to time. The diffusion coefficients calculated using the gravimetric test (see Table 9) are comparable to the results of the permeability test (see Table 11). 
The solubility coefficients vary between each test sample in the gravimetric test (see Table 9); however, they are within the same range. Variability in the solubility coefficient is caused by the effects of casting methods and variability in the thickness of the samples. It is very important to use samples with identical and uniform thickness. Comparison of the solubility and permeability coefficients to the permeability test results is meaningless since the solubility of the sample in the permeability test has a concentration gradient across its thickness due tc the removal of silicone oil from one surface.

The GC tests using end-caps had widely varying results (see Table 5) due to the wide variability in the end-caps. The thickness and mass of the end-caps was not very constant and the use of Biospan to bond the end-caps onto the glass test tubes added material that absorbed more silicone oil and distorted the solubility values. The data from this test were taken at one day periods which missed the transient portion of the curve where the permeant is starting to permeate through the membranes. As a result, no information was obtained that could be used to calculate the diffusion coefficient.

The solubility coefficient of the end-caps was found; however, the variability of the results distorts this value. It is interesting to note that the results from the GC end-cap test correspond well with the solubility values of the gravimetric test (see Figure 38 ). A trend towards decreasing solubility can be seen over several days in the GC end-cap tests. This trend is within the error of the results and may not actually be occurring; however, these types of trends have been seen before in polymers. A decreasing solubility with respect to time may be caused by the extraction of chain extenders, casting solvents and 
unlinked molecules that come loose when the polymer swells with an absorbed permeant. (Comyn, 1985)

The GC sheet test was performed to determine if the method for extraction of silicone oil from Biospan was effective, reproducible and yielded similar results to the gravimetric test. The GC sheet test was successful in that it demonstrated that for extractions of silicone oil from Biospan, DCM was a more effective solvent than hexane (see Table 6). This test also showed that the extractions were repeatable with little error (see Table 6) and that the results were comparable to the results of the gravimetric test.

\subsection{Discussion of Results - Permeability Test}

The permeability tests were performed to acquire values for permeability, diffusion and solubility coefficients for a system modeling the EVAD. These numbers may then be used to indicate the volume of silicone oil that may permeate through the device into the human circulatory system.

As expected, the results of the permeability test showed a constant volume of silicone oil permeating with time once steady state permeation conditions are reached. The three expected regions are seen. The first region where no silicone oil was detected appears as expected. The second region shows a continually increasing rate of permeation up to 24 hours into the test tube (see Figure 40). The third region where the permeation rate has reached steady state is the linear portion of the curve. This portion of the curve is used to calculate the diffusion coefficient and the permeability coefficient. A fourth region can be seen in Figure 39 starting at 8 days. This portion of the curve appears to show a very 
slowly decreasing permeation rate. The reason for the apparent reduced rate of permeation is unknown.

The permeability coefficient was calculated using 11 data points in the linear portion of the curve from 1 to 6 days (see Figure 40). This yielded a permeability coefficient of $4.1 \times 10^{-11} \mathrm{~cm}^{2} / \mathrm{s}$ and a diffusion coefficient of $3.1 \times 10^{-8} \mathrm{~cm}^{2} / \mathrm{s}$. Using these values, the volume of silicone oil permeating through a Biospan diaphragm with an area $60.5 \mathrm{~cm}^{2}$ in one year would be $1.31 \mathrm{ml}$, which is close to the value of $1.75 \mathrm{ml}$ found by Matsuo et. al., (1992). The minimum specified life for the EVAD is two years, and during this time at least $2.62 \mathrm{ml}$ of silicone oil may be lost. The initial volume of silicone oil added to the EVAD when it is assembled is $295 \mathrm{ml}$. The loss of silicone oil over two years is only a $.89 \%$ loss of the total volume of fluid. This should have an insignificant effect on the performance of the device.

The model used to simulate the permeation of silicone oil in the EVAD is believed to take into account many of the influencing factors that effect permeation in an implanted VAD. Some factors which may have resulted in increased apparent permeation of silicone oil in the model over the actual VAD are the use of $1 \mathrm{~cm}$ magnetic stir bars on the inner surface of the diaphragm in the blood and the continuous turbulent stirring of silicone oil. The effects of flucruating pressures and diaphragm flexing were not considered in this study.

Concerns with the test method and extraction procedure for obtaining silicone oil permeation values are discussed in Section 7.1 Test Evaluation. 


\section{Chapter 8: Conclusions \& Recommendations}

The purpose of this thesis was to develop a test method and apparatus that could be used to evaluate the permeability properties of silicone oil (decamethyltetrasiloxane) through a bio-compatible membrane (Biospan - segmented polyether-polyurethane) into blood. This was done to provide some insight into the permeability of polymer membranes for use in circulatory assist devices such as the EVAD developed by World Heart Corporation and the Cardiovascular Devices Division of the University of Ottawa Heart Institute.

This thesis demonstrates a method to evaluate candidate polymers for permeability characteristics while taking into account many of the conditions seen in an implanted circulatory assist device.

Characteristics that were taken into account to model an implanted device include:

1. Temperature. Tests were performed at standard human body temperature of $37^{\circ} \mathrm{C}$.

2. Diaphragm thickness. The thickness of the polymer samples used in the tests were as close as possible to the thickness of the diaphragms currently used in the HSVAD. 
3. Donor fluid. The donor fluid (silicone oil) was the same fluid currently used in the HSVAD.

4. Receiving fluid. The receiving fluid (bovine blood) was the closest fluid to human blood available.

5. Diaphragm boundary conditions. The EVAD is a pulsatile device with continuous flow of blood passing over the diaphragms. In this study, worst case conditions for permeation were assumed and an attempt was made to eliminate any boundary layer effects using magnetic stirring bars in both the donor and receiving fluids.

Additional characteristics which can be analyzed in future tests that may affect permeability characteristics of the diaphragm are the following:

1. Fluctuating stress on diaphragm

2. Diaphragm flexing

3. Fluctuating pressure

4. Changes in molecular structure of the diaphragm due to fatigue, stress cracking, environmental degradation, oxidation and normal aging of the material.

This thesis calculates values for permeability, diffusion and solubility coefficients of the tested polymer. These are not absolute values for the material in question since these results are highly dependant on polymer structure, additives, composition, processing conditions, test apparatus, consistency of donor and receiving fluids.

The permeability of a Biospan polymer diaphragm with dimensions similar to the blood chamber diaphragm in the EVAD is $1.3 \mathrm{ml} /$ year. Assuming that the effects of fluctuating 
stress, flexing, pressure and changing structure of the diaphragm are not substantial this prediction should be accurate.

The results from this thesis provide a test apparatus design and test method that can be used to evaluate the performance of other candidate polymer diaphragms for permeability characteristics of silicone oil through the diaphragm into blood.

\section{Conclusions}

In order to characterize the permeability and diffusion characteristics of the polyurethane, two types of tests were performed.

1) Absorption Tests. To determine the absorption characteristics of the material on its own.

2) Permeability Tests. To determine the permeability characteristics of the material in a system that models an EVAD circulatory assist device.

From these tests several conclusions can be made:

1) The solubility coefficient of silicone oil in a blood/Biospan/silicone oil model of the EVAD is $0.0013 \mathrm{~g} / \mathrm{g}$.

2) The diffusion coefficient of silicone oil in a blood/Biospan/silicone oil model of the EVAD is $3.1 \times 10^{-8} \mathrm{~cm}^{2} / \mathrm{s}$.

3) The permeability coefficient of silicone oil in a blood/Biospan/silicone oil model of the EVAD is $4.1 \times 10^{-11} \mathrm{~cm}^{2} / \mathrm{s}$. 
4) The volume of silicone oil that may be lost through the EVAD blood chamber diaphragm (using the model in this thesis) is $1.3 \mathrm{ml}$ per year assuming worst case turbulent conditions.

5) Silicone oil has a low permeation rate through Biospan.

6) Silicone oil is insoluble in distilled water, hence water is not a suitable receiving medium for permeability tests.

7) Silicone oil is soluble in whole blood and serum.

8) The solubility of silicone oil in whole blood is much higher than silicone oil in serum. Serum is inferior to whole blood for permeability test purposes since serum does not attract silicone oil molecules as well, resulting in depressed permeation rates.

9) The designed permeability test apparatus that models the EVAD demonstrated a permeation rate of $1.3 \mathrm{ml} /$ year for a diaphragm of equal area to the EVAD blood chamber diaphragm. These results were repeatable.

10) In the test model the rate of permeation appeared to decrease after 6 days.

11) It is possible to use whole blood as a receiving fluid in the permeability tests without clotting or major foreign growth.

note: Aggressive mixing and high temperature resulted in lysed RBCs and loss of oxygen from the blood.

12) Degradation of the blood was observed during testing.

13) The extraction procedure listed can be an effective method for extracting silicone oil from blood.

14) Gas Chromatography using Mass Spectrometry for detection can be an effective method for testing blood samples for silicone oil contamination. Good accuracy can be achieved using this method at the concentrations of silicone oil used in these experiments. 
15) The diffusion coefficient found using simple gravimetric tests is similar to the diffusion coefficient in the permeability tests.

16) Small differences in polymer sample thickness, mass, and casting method can result in noticeable changes in solubility and permeability coefficients. The diffusion coefficient appears to be less sensitive to these factors.

17) Silicone oil completely saturates a Biospan sample in under 24 hours.

18) Steady state permeation rate commences at approximately 24 hours.

19) Dichloromethane can be a suitable solvent for extraction of silicone oil from Biospan.

20) Hexane is not as effective at extracting silicone oil from Biospan as dichloromethane

21) Hexane is a better solvent for silicone oil extraction from blood due to its liquid consistency after vigorous mixing and its luwer density than water which, after centrifuging, leaves the solvent on top of the blood parts.

22) Dichloromethane is not a suitable solvent for extraction from blood due to its semisolid consistency after mixing with blood and its higher density than water which places it below the blood parts after centrifuging.

The test results of this thesis show that the goal of the thesis was achieved. A test apparatus was designed and tests were performed yielding useful results that indicate the permeability characteristics of the polymer in question. The test results followed the general trends suggested by Fick's law. The test methods and apparatus are suitable for continued testing on new candidate materials so that a permeability performance comparison can be made. 


\section{Recommendations}

This thesis has proven that it is possible to obtain permeability test results using the previously discussed apparatus and method. During the execution of the tests, several possible improvements were noted which would be beneficial to consider if further research is undertaken.

1) The small magnetic stir bars located inside the tubes touched the surface of the diaphragm during testing. While it was desirable to completely eliminate any boundary conditions on the diaphragm, it was felt that it would be beneficial to avoid contact of the stir bars on the diaphragm.

2) The stirning rate of the magnetic stir bar in the silicone oil reservoir was not monitored. Equipment that could accurately monitor stirring speed would be beneficial.

3) The EVAD operates in a pulsatile fashion. The model would be improved if the flow simulation of silicone oil and blood over the diaphragm surface were also pulsatile.

4) The EVAD has a fluctuating pressure gradient across the diaphragm. Modeling the pressure gradient and the resulting stresses in the diaphragm would improve the model.

5) The blood used in these permeability tests was bovine blood. Bovine blood is not identical to human blood in that bovine blood has more red blood cells. A more accurate model would use human blood if it was available.

6) The test apparatus used an acryiic material for the silicone oil reservoir. This material should be replaced by some other material since the magnetic stir bar caused acrylic wear particles to develop in the silicone oil bath. 
7) During the permeability tests, a fluid (likely water) evaporated out of the blood onto the test tube walls. The volume of blood evaporating should be minimized by reducing the volume of free space between the blood and the top of the tubes.

8) Very little information was available on the materials and casting methods used on the samples in this test. Material characteristics like viscosity (before casting) and casting conditions like temperature, humidity and solvent extraction times should be closely analyzed since these factors affect the polymer at a molecular level.

9) Test diaphragms should have the same number of casting layers as the blood diaphragm used in the EVAD. (3-layer castings were used for the end-caps in this thesis while the blood chamber diaphragms used 4-layer castings. This is due to the more complex shape of the blood chamber and the effects of viscosity of the polymer during casting.)

10) An evaluation of the quality of each sample should be made before it is tested. Characteristics like thickness and mass have been shown to have notable effects on material characteristics.

11) The purity of the silicone oil should be evaluated.

12) There were some indications of reduced permeability after a diaphragm had been tested. This should be investigated as long-term permeability test samples demonstrated a decreasing permeation rate. (This may be due to increased concentration on the receiving side of the diaphragm or an interaction between the silicone oil, blood and polymer.)

13) A study into lamination of the silicone oil side of the diaphragm with a polymer which is resistant to silicone oil would reduce the permeation rate. 


\section{REFERENCES}

Barnhart E.R., (1990): Physicians' Desk Reference, Medical Economics Company Inc., Oradell, N.J., USA.

Bedini R., Chistolini P., De Angelis G., Formisano G., and Caiazza S., (1993): VAD Biomer Blood Sacs: Mechanical Tests and Ultrastructural Observations, Medical Progress Through Technology; 19: 83-88.

Belisle J., Maier S. and Tucker J., (1990): Compositional Analysis of Biomer. Journal of Biomedical Materials Research; 24(12): 1585-1598.

Braunwald E., (1992): Heart Disease, A Textbook of Cardiovascular Medicine $4^{\text {th }}$ Ed., W.B. Saunders Company, Philadelphia, P.A.

Bridges A.J., and Vasey F.B. (1993): Silicone Breast Implants, History, Safety and Potential Complications: Arch Intern Med; 153: 2638-2644.

Capek P., Kadipasaoglu K.A., Radovancevic B., Furusho N., Clubb F.J., Myers T.J., Duncan J.M., McAllister H.A., and Frazier O.H., (1992): Human Intraperitoneal Response to a Left Ventricular Assist Device with a Ti-6Al-4V Alloy Surface: ASAIO Joumal; 38: M543-M549.

Comstock M.J., (1982): Biomaterials: Interfacial Phenomena and Applications, American Chemical Society, Washington, D.C..

Comyn K., (1985): Polymer Permeabiliiy, Elsevier Applied Science Publishers, New York.

Crank J., and Park G.S., (1968): Diffusion in Polymers, Academic Press, London \& New York.

Diegel P.D., Mussivand T., Holfert J.W., Nahon D., Miller J., Maclean G.K., Santerre J.P., Bearnson G.B., Juretich J., Hansen A.C. and Olsen D.B., (1991):

Electrohydraulic Ventricular Assist Device Development: ASAIO Transactions 37; M206-M207.

Diegel P.D., Mussivand T., Holfert J.W., Juretch J.T., Miller J., Maclean G.K., Szurmak Z., Santerre J.P., Rajagopalan K., Dew P.A., Hansen A.C. Sinnott M.M., Olsen 
D.B. and Keon W., (1992): Electrohydraulic Ventricular Assist Device Development: ASAIO Transactions 38; M306-M310.

Felix K., Janz S., Pitha J., Williams J.A., Mushinski E.B., Bornkamm G.W., and Potter M., (1996): Cytotoxicity and Membrane Damage In Vitro by Inclusion Complexes Between Gamma-cyclodextrin and Siloxanes: Current Topics in Microbiology \& Immunology; 210: 93-99.

Flynn G.L. and Smith E.W., (1971): Membrane Diffusion I: Design and Testing of a New Multifeatured Diffusion Cell: Journal of Pharmaceutical Sciences; 60(11): 17131717.

Fujimoto L., Chen J., Smith W.A. and Kiraly R.J., (1989): A Simple and Compact Total Artificial Heart for Temporary Use: ASAIO Transactions 35: 260-262.

Gent A.N. and Tobias R.H., (1982): Diffusion and Equilibrium Swelling of Macromolecular Networks by their Linear Homologs: Joumal of Polymer Science: Polymer Physics Edition; 20: 2317-2327.

Glass E.J., (1989): Polymers in Aqueous Media, American Chemical Society, Washington, D.C..

Hill D.J., (1989): Bridging to Cardiac Transplantation: Annals of Thoracic Surgery; 47: $167-71$

Hill D.J., (1993): How Much Do We Need to Know Before Approving a Ventricular Assist Device?: Annals of Thoracic Surgery; 55: 314-328.

Hu M. and Matheson L.E., (1993): The Development of a Predictive Method for the Estimation of Flux Through Polydimethylsiloxane Membranes. III. Application to a Series of Substituted Pyridines. Pharmaceutical Research, 10(5), 732-36.

Hunke W.A., and Matheson L.E., (1981): Mass Transport Properties of Co(polyether) Polyurethane Membranes II: Permeability and Sorption Characteristics: Journal of Pharmaceutical Sciences; 70(12): 1313-18.

Iordanskii A.L., Rudakova T.E., and Zaikov G.E., (1994): Interaction of Polymers with Bioactive and Corrosive Media, Koninklijke Wohrmann bv, Zutphen, The Netherlands.

Irie H., Massiello A., Kiraly R., Chen J., Crouch R., Fukamachi K., Jacobs G., Harasaki H., Golding L., Thomas D., Carriker W., and Butler K., (1992): Initial In-Vivo 
Tests of an Electrohydraulic Actuated Total Artificial Heart: ASAIO Journal 38: M497-M500.

Kala S.V., Lykissa E.D., and Lebovitz R.M., (1997): Detection and Characterization of Poly(dimethylsiloxane)s in Biological Tissues by GC/AED and GC/MS:

Analytical Chemistry; 69(7): 1267 - 1272.

King M., Personal Interview, (1996).

Leonard P., (1992): Crack-Resistant Polycarbonate Urethane Polymer Prostheses. United States Patent \#5,133,742.

Liu R, and Matheson L.E., (1994): Comparative Molecular Field Analysis Combined With Physicochemical Parameters for Prediction of Polydimethylsiloxane Membrane Flux in Isopropanol. Pharmaceutical Research, 11(2), 257-66.

Macoviak J.A., Dasse K.A. and Poirier V.L., (1990): Mechanical Cardiac Assistance and Replacement. Cardiology Clinics, 8(1), 39-53.

Marieb E.N., (1995): Human Anatomy And Physiology, Third Edition, The Benjamin/Cummings Publishing Company, Inc., Don Mills, Ontario, Canada.

Matheson L.E. and Hu M., (1993): The Development of a Predictive Method for the Estimation of Flux Through Polydimethylsiloxane Membranes. IV. Application to a Series of Substituted Quinolines. Pharmaceutical Research, 10(6), 839-42.

Matsuo Y., Taenaka Y., Takano H., Nakatani T., Tatsumi E., Masuzawa T., Goto M., Yagura A., Akagi H., Sasaki E., Inoue K., Sakaki M., Baba Y., Akutsu T., Ohumi T., Tsukahara K., Tsuchimoto K., and Tanaka I., (1991): Serum Silicon Levels in Recipients of an Electrohydraulic Ventricular Assist System: ASAIO Transactions, 37: M310-M311.

Matsuo Y., Taenaka Y., Takano H., Nakatani T., Tatsumi E., Masuzawa T., Goto M., Yagura A., Akagi H., Sasaki E., Inoue K., Sakaki M., Baba Y., Akutsu T., Ohumi T., Tsukahara K., Tsuchimoto K., and Tanaka I., (1992): Permeation of Silicone Oil Through a Diaphragm in an Electrohydraulic Ventricular Assist System: ASAIO Journal, 38: M570-M573.

Matsuyama H., Kariya A. and Teramoto M., (1994): Effect of Siloxane Chain Lengths of Monomers on Characteristics of Pervaporation Membranes Prepared by Plasma Polymerization: Journal of Applied Polymer Sciences, 51: 689-693. 
McCarthy P.M., (1995): HeartMate Implantable Left Ventricular Assist Device: Bridge to Transplantation and Future Applications: Annals of Thoracic Surgery 59: S46-51

McMillin C.R., (1983): Physical Testing of Elastomers for Cardiovascular Applications. Artificial Organs; 7(1):78-91.

McMillin C.R., (1987): Development of Tests to Evaluate Candidate Elastomers for Artificial Heart Diaphragms. Artificial Organs; 11(5):395-404.

McMillin C.R., Fishback T., Harper T., Mussivand T., Kiraly R., Smith W., Navarro R., and Nose Y., (1989): Development of Compliance Chamber Diaphragms with Reduced Permeability. Journal of Biomedical Materials Research, vol. 23, 117128.

McMillin C.R., (1994): Elastomers for Biomedical Application. Biomedical Applications; Vol. 67:417-46.

Miller I. and Freund J.E., (1985): Probability and Statistics for Engineers, Third Edition: Prentice-Hall, Toronto, Canada.

Mulder M., (1991): Basic Principles of Membrane Technology, Kluwer Academic Publishers, Norwell, MA U.S.A..

Pauley J.L., and Ghanem A., (1990): Diffusion Evaluation of Decamethyltetrasiloxane and Water Through a Static Biomer Membrane, Proprietary Literature EVAD document \#1078-06-00

Pauley J.L., and Ghanem A., (1991): First Follow-up on the Diffusion Study of Biomer and Decamethyltetrasiloxane, Proprietary Literature EVAD document \#1 107-0600

Peterson T., Burton S., Englund B., and Grosh S., (1990): In-vitro Permeability of Poly(Ethylene-Vinyl Acetate) and Microporous Polyethylene Membranes; Proceedings of the $17^{\text {th }}$ Annual International Symposium on Control Release of Bioactive Materials., p 411.

Ramasamy M., Phillips L., Chen H., Miller P.J., O'Campo A., Green B.A., Oyer P.E., Jassawalla J.S., and Portner P.M., (1989): Long-Term In-Vivo Evaluation of Volume Compensator for a Left Ventricular Assist System: $15^{\text {th }}$ Annual Meeting of the Society for Biomaterials April 28 - May 2.

Reed A.M., Potter J., and Szycher M., (1994): A Solution Grade Biostable Polyurethane Elastomer: ChronoFlex AR: Journal of Biomaterials Applications, 8:210-236. 
Reid J., Rosenberg G. and Pierce W., (1985): Transmission of Water Through a Biocompatible Polyurethane: Application to Circulatory Assist Devices: Journal of Biomedical Materials Research, Vol. 19, 1881-1202.

Rowles J., Khanwilkar P., Diegel P., Hansen A., Bearnson G., Smith K., Tatsumi E., and Olsen D., (1992): Development of a Totally Implantable Artificial Heart. ASAIO Journal; 38: M713 - 716 .

Rowles J.R., Mortimer B.J. and Olsen D.B., (1993): Ventricular Assist and Total Artificial Heart Devices for Clinical Use in 1993: ASAIO Journal: 840-855.

Santerre J.P., Labow R.S., and Adams G.A., (1993): Enzyme-Biomaterial Interactions: Effect of Biosystems on Degradation of Polyurethanes: Journal of Biomedical Materials Research, 27:97-109.

Sapirstein J.S., Pae W.E., Rosenberg G., and Pierce W.S., (1994): The Development of Permanent Circulatory Support Systems: Seminars in Thoracic and Cardiovascular Surgery 6(3): 188-194.

Tsuruta T., Hayashi T., Kataoka K., Ishihara K. and Kimura Y., (1993): Biomedical Applications of Polymeric Materials, London Press, London, England.

Ward R.S., Litwak P., White K.A., Robinson J., Yilgor I., and Riffle J.S., (1987): BPS215M: A New Polyurethaneurea for Biomedical Devices: Development and In Vitro Testing in the Pierce-Donachy VAD: $13^{\text {th }}$ Annual Meeting of the society for Biomaterials June 2-6, 1987: p 259.

Webster H.F. and Wightman J.P., (1991): Characterization of Thin Films of Poly(dimethylsiloxane) formed from Surface Diffusion Across Defined Polymer Substrates: Langmuir 7(12): 3099-3109.

Wesslen B., Kober M,Freij-Larsson C., Ljungh A., and Paulsson M., (1994): Protein in Adsorption of Poly(ether urethane) Surfaces Modified by Amphiphilic and Hydrophitit Polymers: Biomaterials, 15(4):278-84.

Williams W.J., Beutler E., Erslev A.J., and Lichtman M.A., (1990): Hematology, Fourth Edition, McGraw-Hill, Inc., New York, USA.

Wilson G.J., MacGregor D.C., Klement P., Dereume J., Weber B.A., Binnington A.G., and Pinchuk L., (1991): The Composite Corethane/Dacron Vascular Prosthesis: ASAIO Transactions, 37: M475-M476. 
Yang M.J., Deng X.Y and Guidoin R., (1996): Water Permeability of Poly'rrethane Membranes: Fifth World Biomaterials Congress May 29 - June 2, 1996, Toronto Canada, p. 820.

Yeh J., Gordon B. and Rosenberg G., (1994): Moisture Diffusivity of Biomer versus Biomer-Coated Polyisobutylene Polyurethane Urea (PIB-PUU): A Potential Blood Sac Material for the Artificial Heart. Journal of Materials Science Letters, Vol. 13, 1390-1391.

Yeh J.T., Gordon B. and Rosenberg G., (1994): PIB-PUU Linear Multiblock Copolymer: A New Blood Sac Material for the Penn State Artificial Heart: $20^{\text {th }}$ Annual Meeting of the Society for Biomaterials, p. 47.

Chronoflex AR - Product Literature, 1996: CardioTech International; Woburn, Ma, USA.

ChronoThane B - Product Literature, 1996: CardioTech International; Woburn, Ma, USA.

Biospan - Product Literature, 1996: The Polymer Technology Group; Emeryville, Ca, USA.

HeartSaver VAD, 1997 - Product Literature: World Heart Corporation; Ottawa, On, Canada. 


\section{APPENDIX A:}

Experimental Apparatus 


\section{Appendix A: Experimental Apparatus}

The test equipment used in these experiments was acquired from stock in the labs at the Cardiovascular Devices Division of the University of Ottawa Heart Institute. The test apparatus materials were purchased from Canus Plastics, Ottawa, Ontario, Canada. Machining and assembly were performed in the machine shops at Carleton University, Ottawa, Ontario, Canada. The glassware and extraction solvents were purchased through VWR Canada. The whole bovine blood was obtained through the Cardiovascular Devices Division of the University of Ottawa Heart Institute and the biomaterial, Biospan, was obtained through the manufacturer.

\section{Appendix A.1 Experimental Apparatus - Test Evaluation}

\section{Non disposable:}

Magnetic stir bars 15 PTFE coating ( $10 \mathrm{~mm} \times 3 \mathrm{~mm}$ )

see Figure 18

Pipet $20 \mathrm{ml}$ glass $+-.06 \mathrm{ml}$

$40 \mathrm{ml}$ glass scintillation vials

see Figure 19 .

Tweezers

Sharpie fine point permanent marker

Sanford

Hamilton Standard $10 \mu \mathrm{l}$ injection syringe

see Figure 20

Disposable:

Glass sample vials, $1.5 \mathrm{ml}$

see Figure 20

Caps w/PTFE liners

$10 \mathrm{cc}$ syringe

Becton Dickinson \& Company

see Figure 21

22G1 1/2 Precision Glide Needle

see Figure 21

Becton Dickinson \& Company

Pasteur Pipettes 9 inch

Maple Leaf Brand

\section{Apparatus:}

Magnetic Stir Tray

Thermix Stirrer Model 220T, Fisher Scientific

Turn Table

Chuo-Denki 8002A semi-automatic direct drive

see Figure 22

see Figure 23

Centrifuge 
Sorvall RT6000B Refrigerated Centrifuge

\section{Solutions:}

Ethyl Alcohol $95 \%, \mathrm{CH}_{3} \mathrm{CH}_{2} \mathrm{OH}$ (cleaning fluid)

Hexane, $\mathrm{C}_{6} \mathrm{H}_{14}, \mathrm{FW} 86.18$ (extraction solvent)

HPLC Grade

EM Science

CAS 110-54-3

Dichloromethane or Methylene Chloride, $\mathrm{CH}_{2} \mathrm{Cl}_{2}, \mathrm{FW} 84.94$ (extraction solvent)

HPLC Grade

EM Science

CAS 75-09-2

Decamethyltetrasiloxane, $\mathrm{C}_{10} \mathrm{H}_{30} \mathrm{O}_{3} \mathrm{Si}_{4}$, FW 310.69, (Silicone Oil)

United Chemical Technologies, Inc. Bristol, PA, USA

CAS 141-62-8

Whole Bovine Blood

see Figure 21

anticoagulant Citrate Phosphate Dextrose Adenine solution (CPDA)

$450 \mathrm{ml}$ whole bovine blood $+63 \mathrm{ml} \mathrm{CPDA}$

$63 \mathrm{ml}$ of CPDA contains

$188 \mathrm{mg}$ citric acid (anhydrous)

$1.66 \mathrm{~g}$ sodium citrate (dihydrate)

$140 \mathrm{mg}$ monobasic sodium phosphate (monohydrate)

$2.01 \mathrm{~g}$ dextrose (monohydrate)

$17.3 \mathrm{mg}$ adenine

\section{Appendix A.2 Experimental Apparatus - Absorption Test}

\section{Non disposable:}

Thermometer -20 to $110^{\circ} \mathrm{C}$

see Figure 22

Acrylic test setup

see Figure 22

manufactured

Glass tubes 1 in $O D, 1 / 16$ in wall thickness

see Figure 24

manufactured

Test cap casting molds, aluminum

Flat casting mold, aluminum

Tygon tubing

Tie straps

PTFE Stoppers

Magnetic stir bars 15 PTFE coating (10 mm x $3 \mathrm{~mm}$ )

Magnetic stir bar 1 PTFE coating ( $70 \mathrm{~mm} \times 13 \mathrm{~mm}$ )

Pipet $10 \mathrm{ml}$ glass.$+ .06 \mathrm{ml}$

$40 \mathrm{ml}$ glass scintillation vials w/PTFE liners

$500 \mathrm{ml}$ clear glass bottle

see Figure 24

see Figure 24

see Figure 18

see Figure 18

see Figure 25

see Figure 19

see Figure 26 
Electrical clips

see Figure 27

Tweezers

Hamilton Standard $10 \mu l$ injection syringe

see Figure 20

Modified polypropylene centrifuge tubes

Sharpie fine point permanent marker

Sanford

Disposable:

Glass sample vials, $1.5 \mathrm{ml}$

see Figure 20

Caps w/PTFE liners

see Figure 20

$10 \mathrm{cc}$ syringe

Becton Dickinson \& Company

22G1 1/2 Precision Glide Needle

see Figure 21

Becton Dickinson \& Company

see Figure 21

Non-Latex Medical Examination Gloves (L)

Maxxim Medical

Pasteur Pipettes 9 inch

Maple Leaf Brand

\section{Apparatus:}

Gravity Convection Incubator

Economy model 2EG, Precision

see Figure 22

Oven

Thermocenter Salvis

Magnetic Stir Tray

see Figure 22

Thermix Stirrer Model 220T, Fisher Scientific

Centrifuge

Sorvall RT6000B Refrigerated Centrifuge

Scale

Mettler AJ100 +- .0001g

\section{Solutions:}

Ethyl Alcohol $95 \%, \mathrm{CH}_{3} \mathrm{CH}_{2} \mathrm{OH}$ (cleaning fluid)

Hexane, $\mathrm{C}_{6} \mathrm{H}_{14}$, FW 86.18 (extraction solvent)

HPLC Grade

EM Science

CAS 110-54-3

Dichloromethane or Methylene Chloride, $\mathrm{CH}_{2} \mathrm{Cl}_{2}, \mathrm{FW} 84.94$ (extraction solvent)

HPLC Grade

EM Science

CAS 75-09-2

Decamethyltetrasiloxane, $\mathrm{C}_{10} \mathrm{H}_{30} \mathrm{O}_{3} \mathrm{Si}_{4}$, FW 310.69, (Silicone Oil)

United Chemical Technologies, Inc. Bristol, PA, USA 
CAS $141-62-8$

Whole Bovine Blood

see Figure 21

anticoagulant Citrate Phosphate Dextrose Adenine solution (CPDA)

$450 \mathrm{ml}$ whole bovine blood $+63 \mathrm{ml}$ CPDA

$63 \mathrm{ml}$ of CPDA contains

$188 \mathrm{mg}$ citric acid (anhydrous)

$1.66 \mathrm{~g}$ sodium citrate (dihydrate)

$140 \mathrm{mg}$ monobasic sodium phosphate (monohydrate)

$2.01 \mathrm{~g}$ dextrose (monohydrate)

$17.3 \mathrm{mg}$ adenine

Biospan - Segmented Polyurethane (SPU)

see Figure 28,29,30

The Polymer Technology Group, Incorporated, Emeryville, CA, USA

\section{Appendix A.3 Experimental Apparatus - Permeability Test}

\section{Non disposable:}

Thermometer -20 to $110^{\circ} \mathrm{C}$

see Figure 22

Acrylic test setup

manufactured

Glass tubes 1 in OD, 1/16 in wall thickness

manufactured

see Figure 22

see Figure 24

Test cap casting molds, aluminum

Tygon tubing

see Figure 24

Tie straps

see Figure 24

PTFE Stoppers

Magnetic stir bars 15 PTFE coating (10 mm x $3 \mathrm{~mm}$ )

see Figure 18

see Figure 18

Magnetic stir bar 1 PTFE coating (70 $\mathrm{mm} \times 13 \mathrm{~mm}$ )

Pipet $20 \mathrm{ml}$ glass $+-.06 \mathrm{ml}$

$40 \mathrm{ml}$ glass scintillation vials w/PTFE liners

Tweezers

Modified polypropylene centrifuge tubes

Hamilton Standard $10 \mu l$ injection syringe

Sharpie fine point permanent marker

see Figure 25

see Figure 19

Sanford

Disposable:

Glass sample vials, $1.5 \mathrm{ml}$

see Figure 20

Caps w/PTFE liners

see Figure 20

$10 \mathrm{cc}$ syringe

Becton Dickinson \& Company

22G1 1/2 Precision Glide Needle

see Figure 21

Becton Dickinson \& Company

see Figure 21

Non-Latex Medical Examination Gloves (L) 


\section{Maxxim Medical \\ Pasteur Pipettes 9 inch \\ Maple Leaf Brand}

\section{Apparatus:}

Gravity Convection Incubator

see Figure 22

Economy model 2EG, Precision

Oven

Thermocenter Salvis

Magnetic Stir Tray

see Figure 22

Thermix Stirrer Model 220T , Fisher Scientific

Turn Table

see Figure 23

Chuo-Denki 8002A semi-automatic direct drive

Centrifuge

Sorvall RT6000B Refrigerated Centrifuge

Scale

Mettler AJ100 +- .0001g

Digital Depth Gauge (dial comparator stand)

\section{Solutions:}

Ethyl Alcohol 95\%, $\mathrm{CH}_{3} \mathrm{CH}_{2} \mathrm{OH}$ (cleaning fluid)

Hexane, $\mathrm{C}_{6} \mathrm{H}_{14}, \mathrm{FW} 86.18$ (extraction solvent)

HPLC Grade

EM Science

CAS 110-54-3

Decamethyltetrasiloxane, $\mathrm{C}_{10} \mathrm{H}_{30} \mathrm{O}_{3} \mathrm{Si}_{4}, \mathrm{FW} \mathrm{310.69,} \mathrm{(Silicone} \mathrm{Oil)}$

United Chemical Technologies, Inc. Bristol, PA, USA

CAS 141-62-8

Whole Bovine Blood

anticoagulant Citrate Phosphate Dextrose Adenine solution (CPDA)

$450 \mathrm{ml}$ whole bovine blood $+63 \mathrm{ml}$ CPDA

$63 \mathrm{ml}$ of CPDA contains

$188 \mathrm{mg}$ citric acid (anhydrous)

$1.66 \mathrm{~g}$ sodium citrate (dihydrate)

$140 \mathrm{mg}$ monobasic sodium phosphate (monohydrate)

$2.01 \mathrm{~g}$ dextrose (monohydrate)

$17.3 \mathrm{mg}$ adenine

Biospan - Segmented Polyurethane (SPU)

see Figures 28,29,30

The Polymer Technology Group, Incorporated, Emeryville, CA, USA 


\section{APPENDIX B:}

Figures 
Figure 1: Circulatory System Diagram: Marieb, (1995)

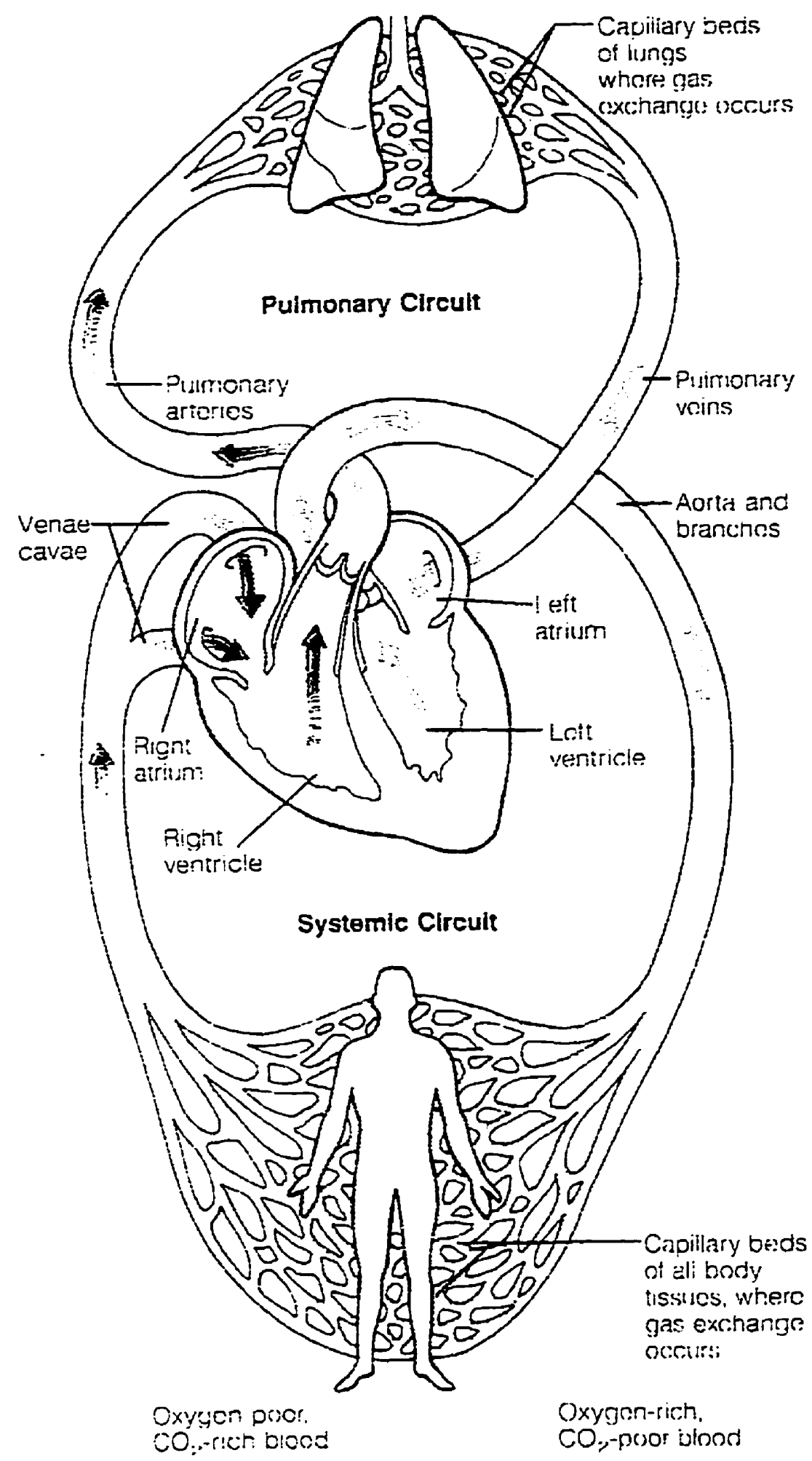


Figure 2: Heart Crossection - Marieb, (1995)

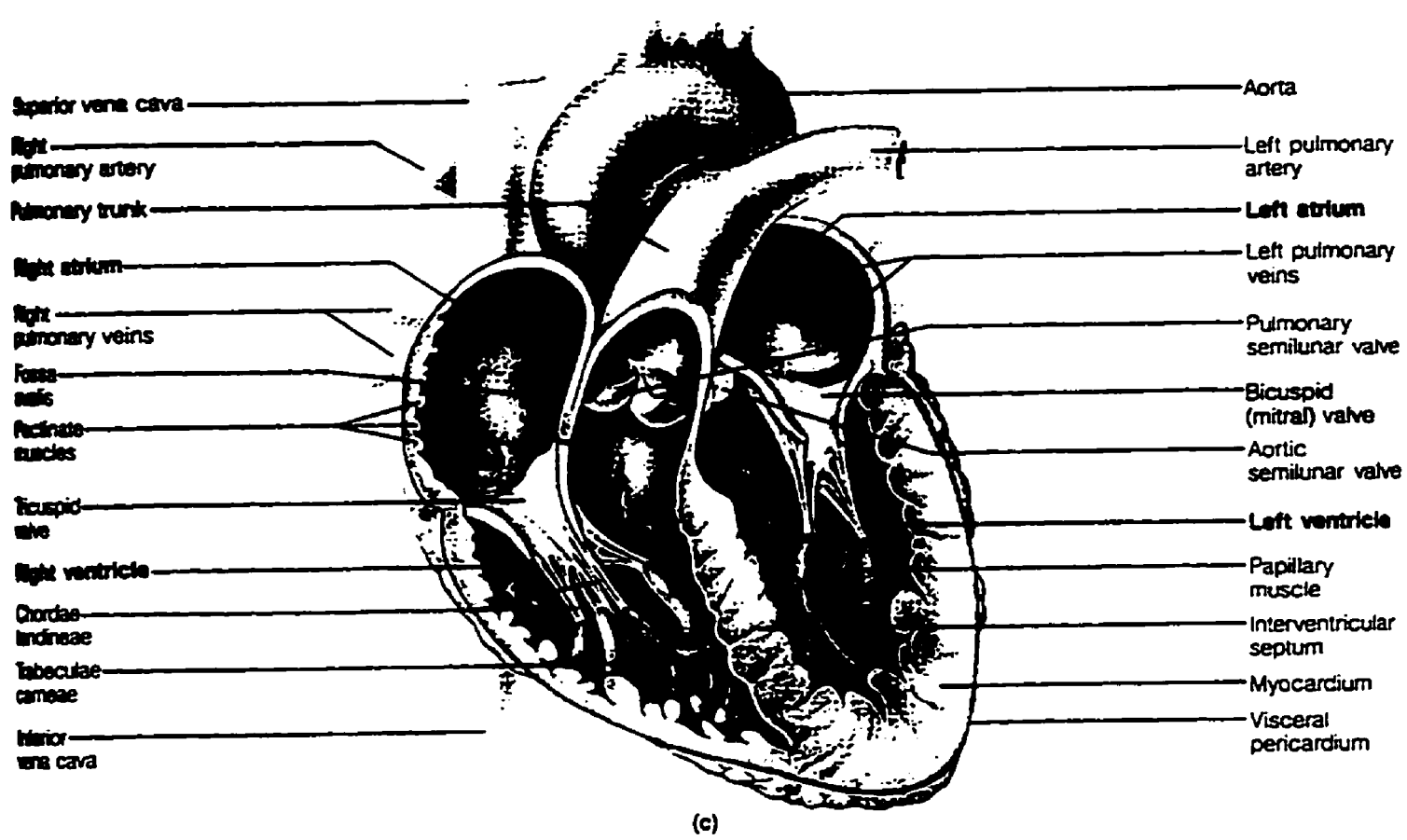

Figure 3: Heart Valves - Marieb, (1995)

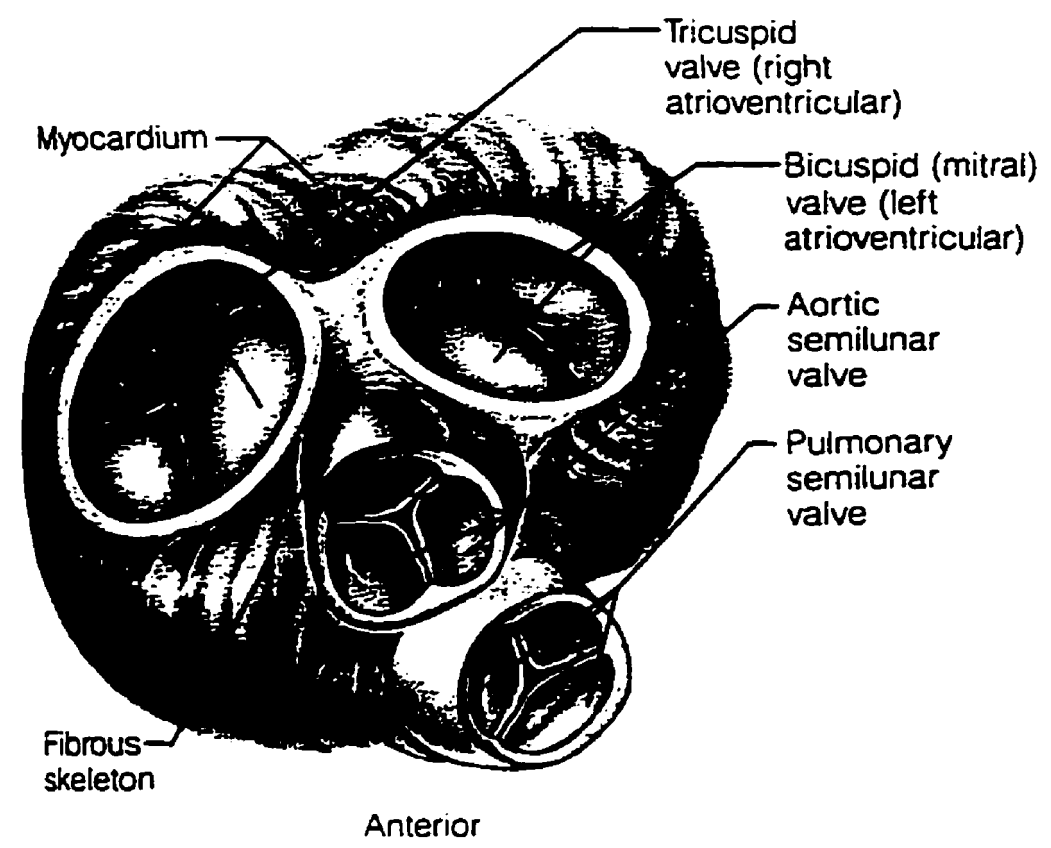


Figure 4: Vessel Diagram - Marieb, (1995)

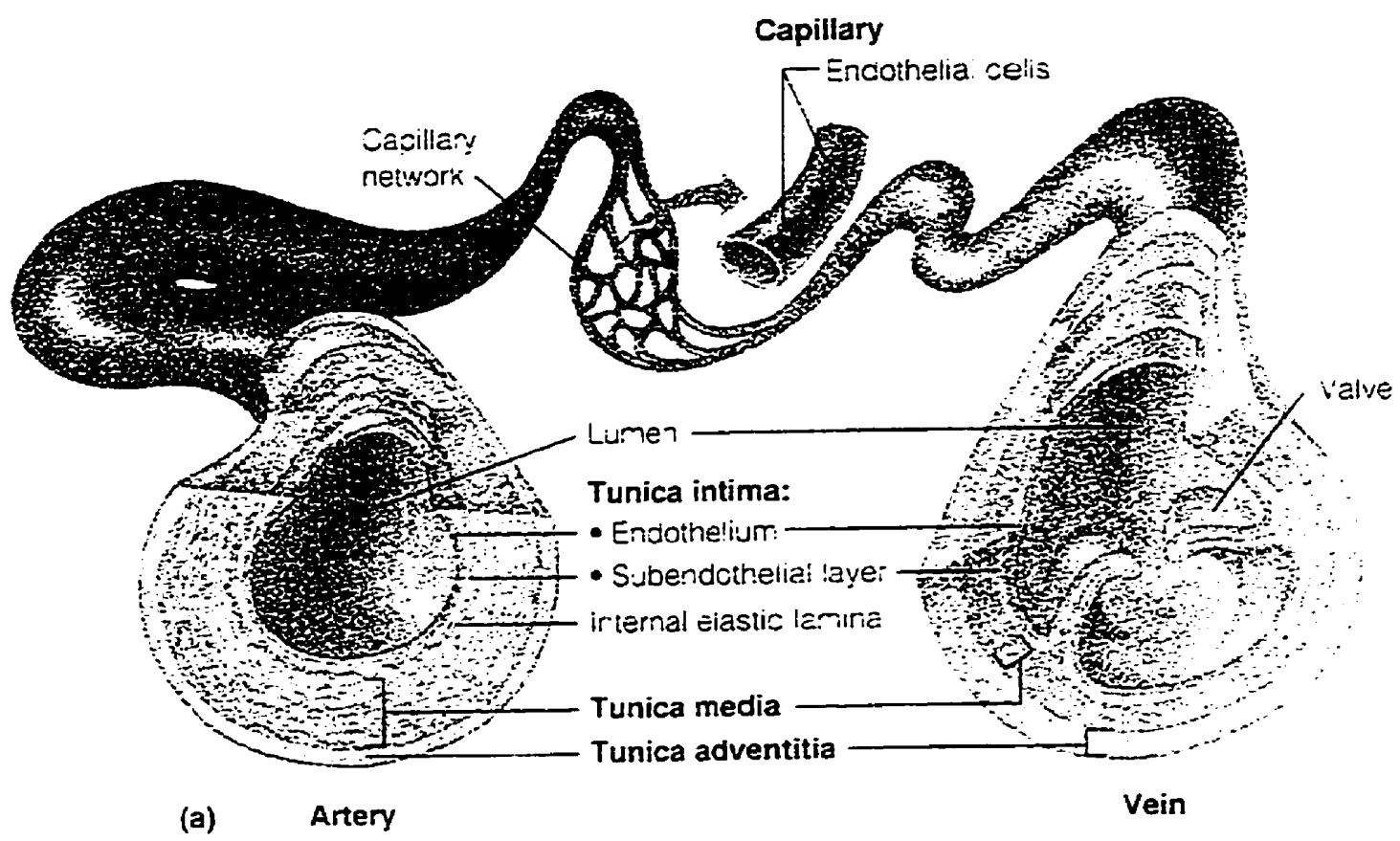


Figure 5: HSVAD Location in Body

World Heart Corporation Product Literature

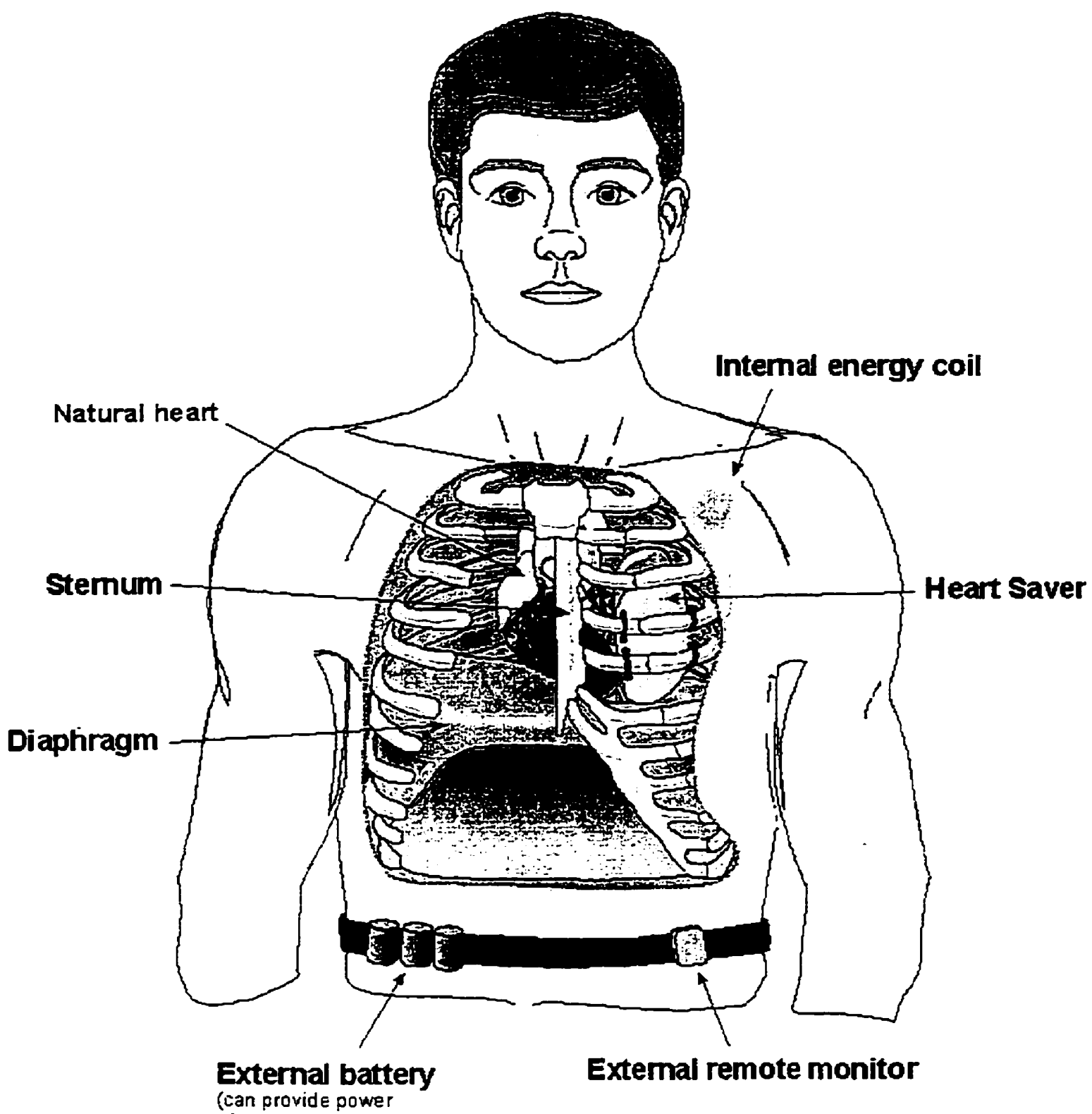

for up to 8 nours) 
Figure 6: HSVAD Connection Diagram

World Heart Corporation Product Literature

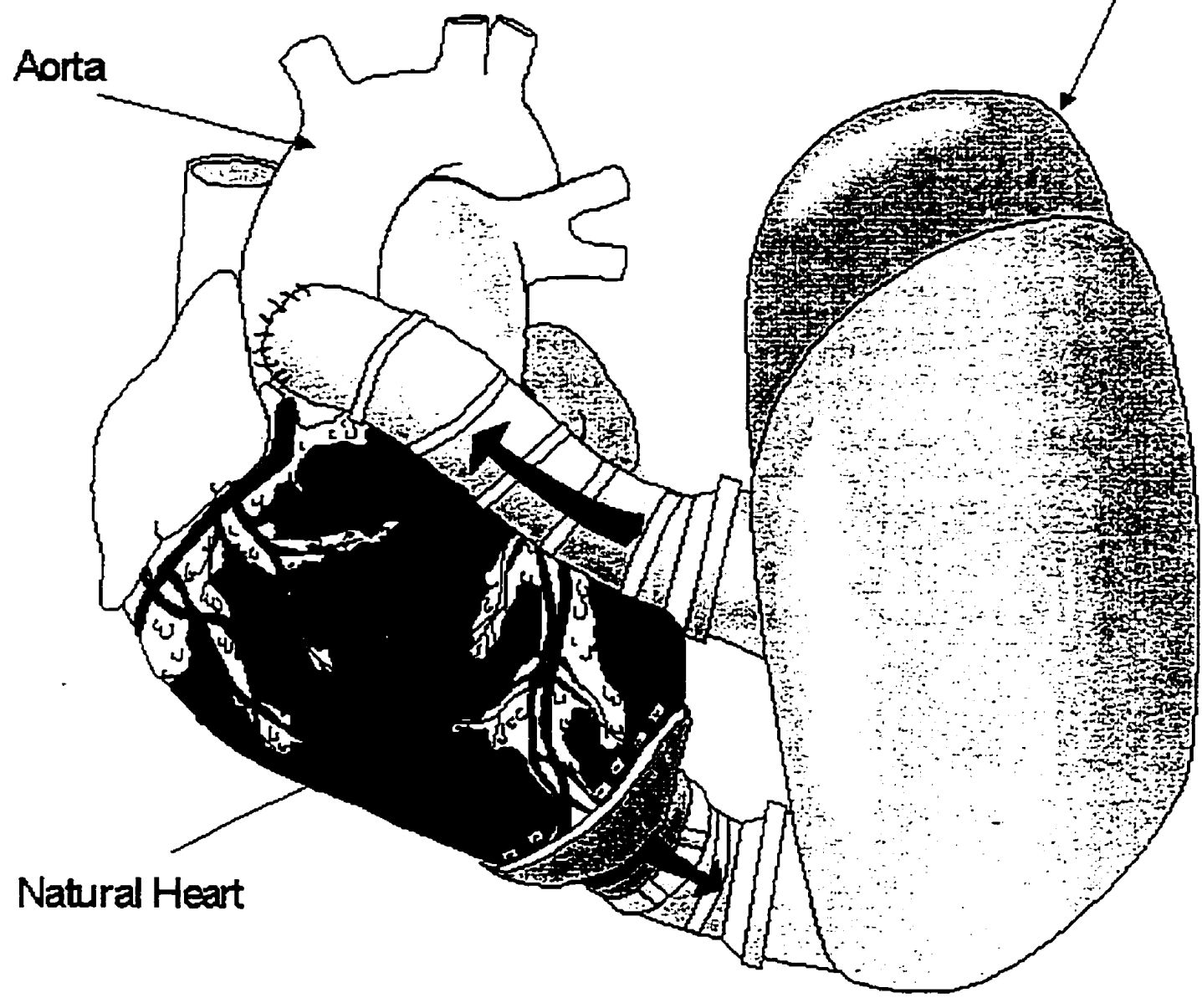


Figure 7: Blood Chamber and VDC Diaphragm Placement

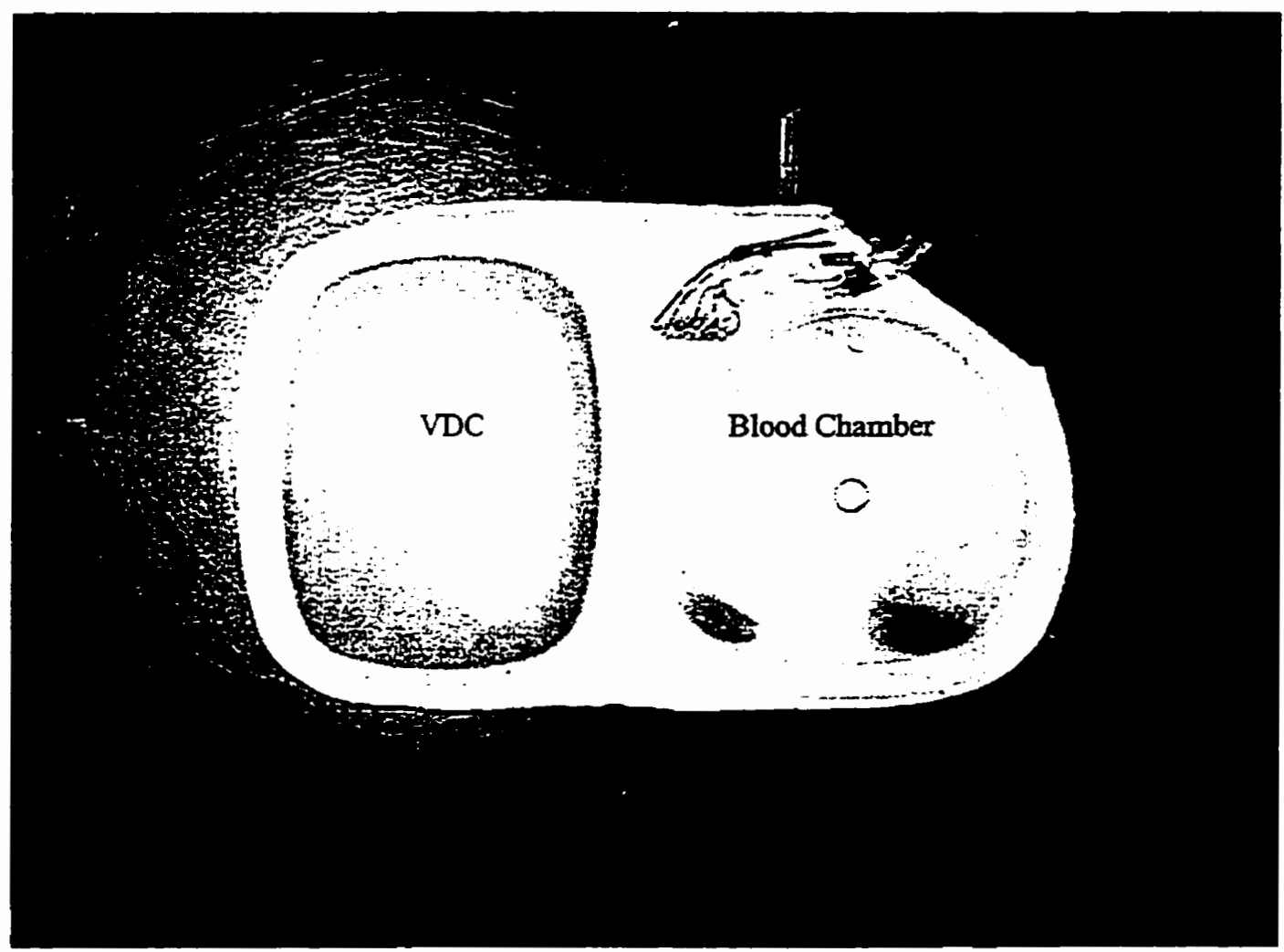

Figure 8: Blood Chamber Diaphragm

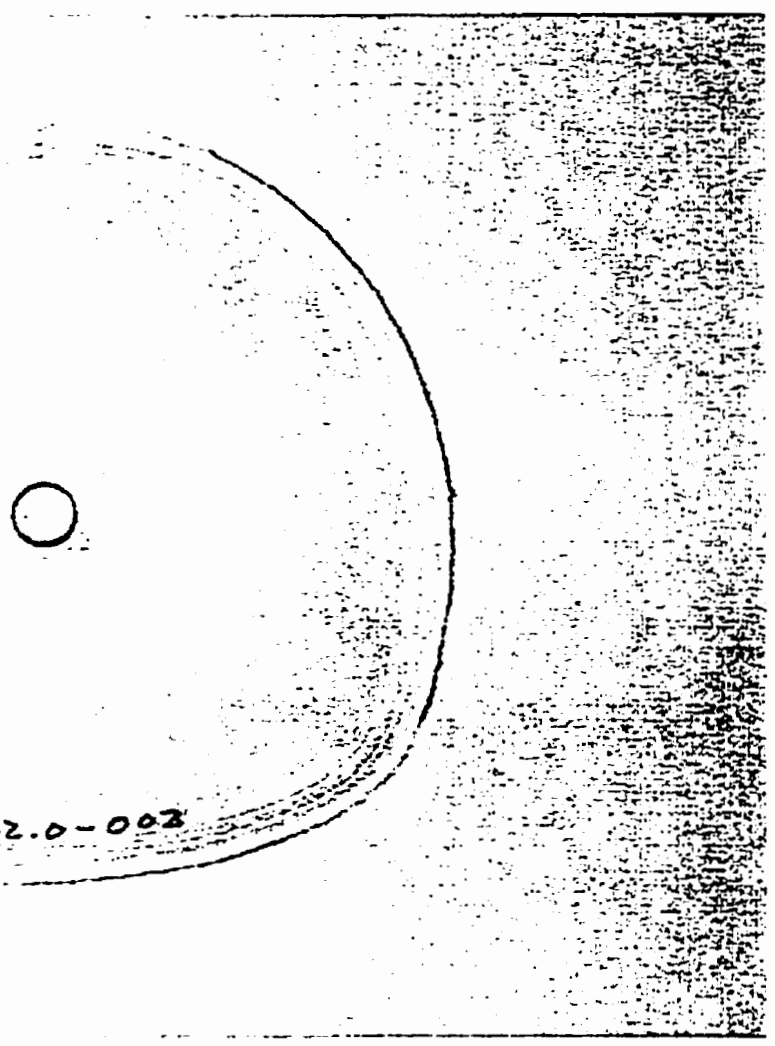


Figure 9: Unassembled Device

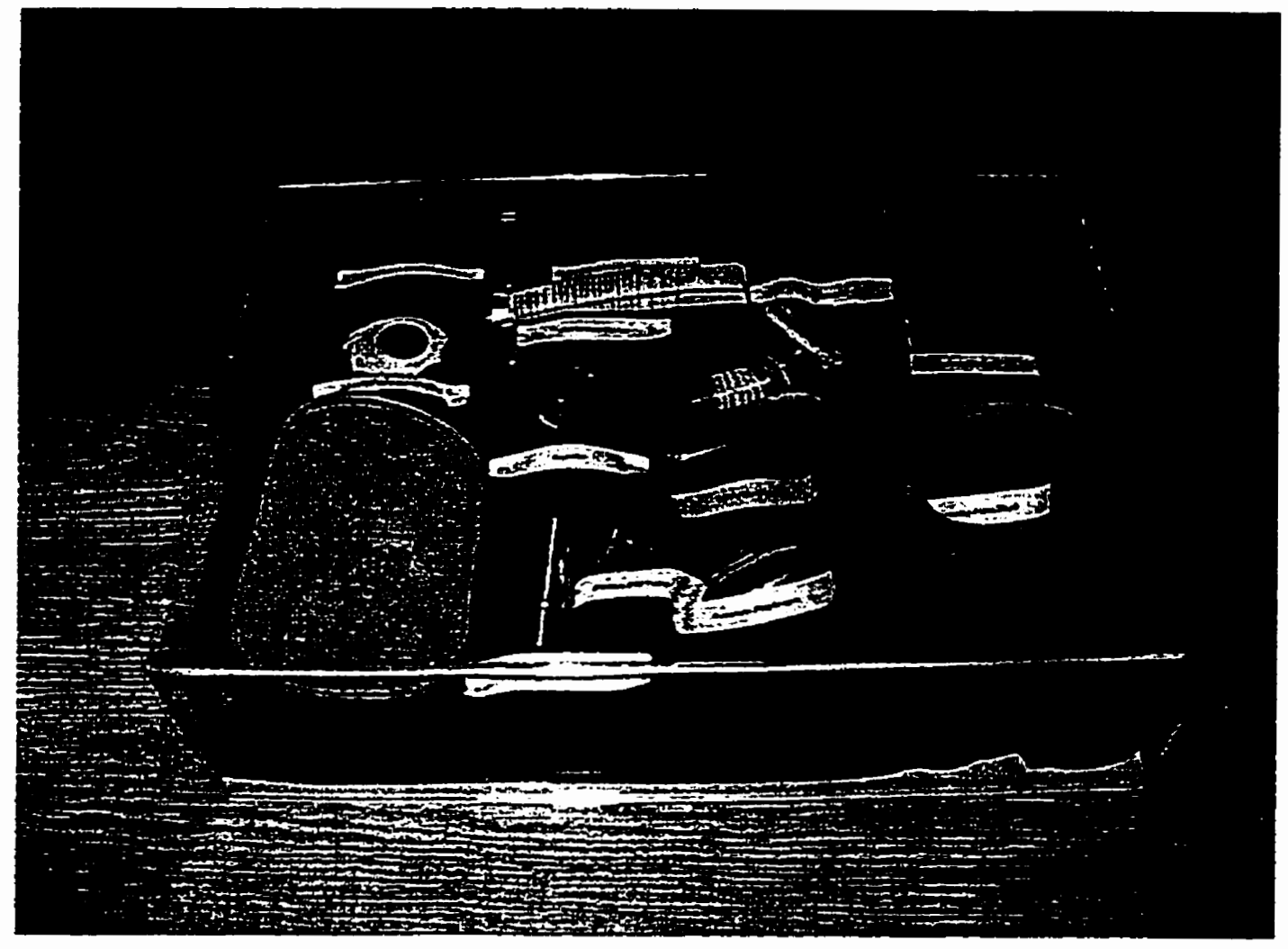

\section{Figure 10: VDC Diaphragm}

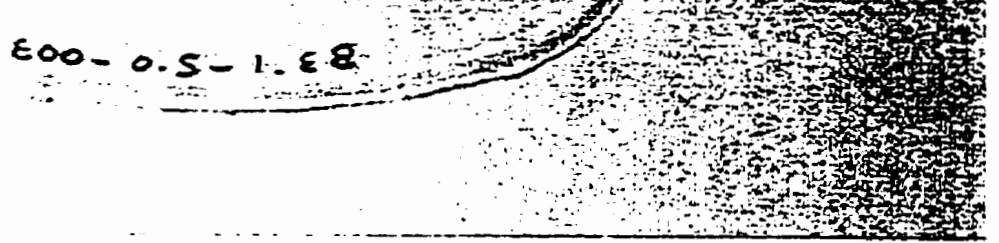


Figure 11: Assembly Drawing of Permeability Test Apparatus

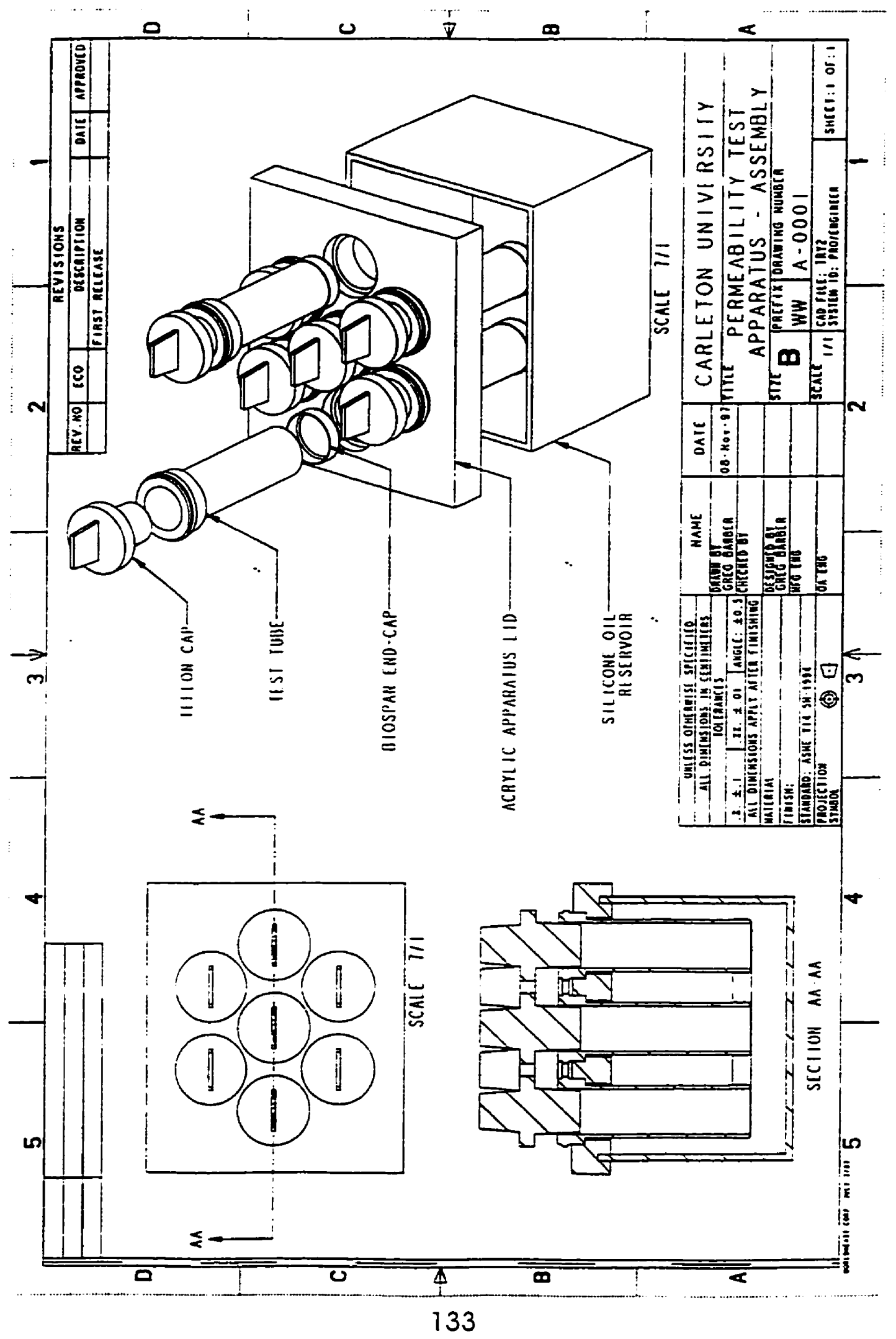


Figure 12: Biospan Molecule
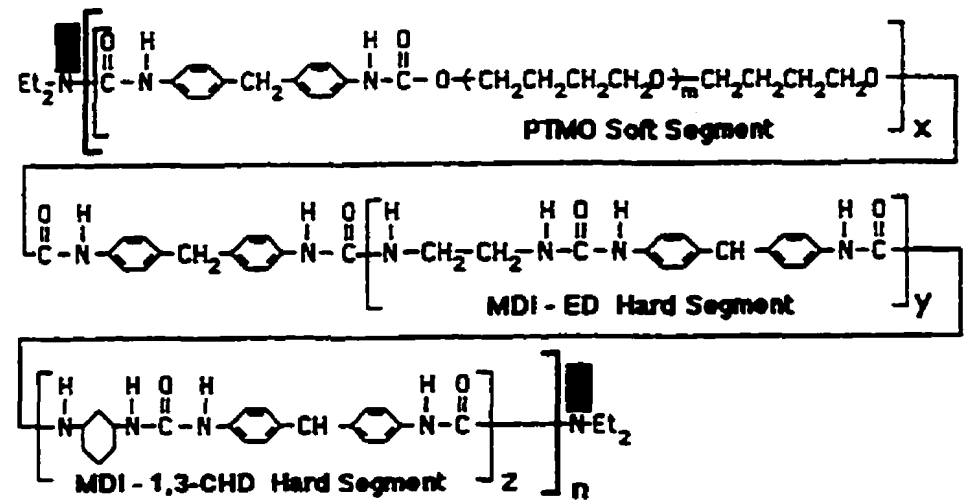

FGUPE 1. Ideafiad chomical stucture of

Biomere and BioSpane segmented potyurethanes

Figure 13: Decamethyltetrasiloxane - Silicone Oil<smiles>C[Si](C)(C)O[Si](C)(C)O[Si](C)(C)O[Si](C)(C)C</smiles> 
Figure 14: Permeation Through a Film - Curve Approaching Steady State: Comyn, (1985)

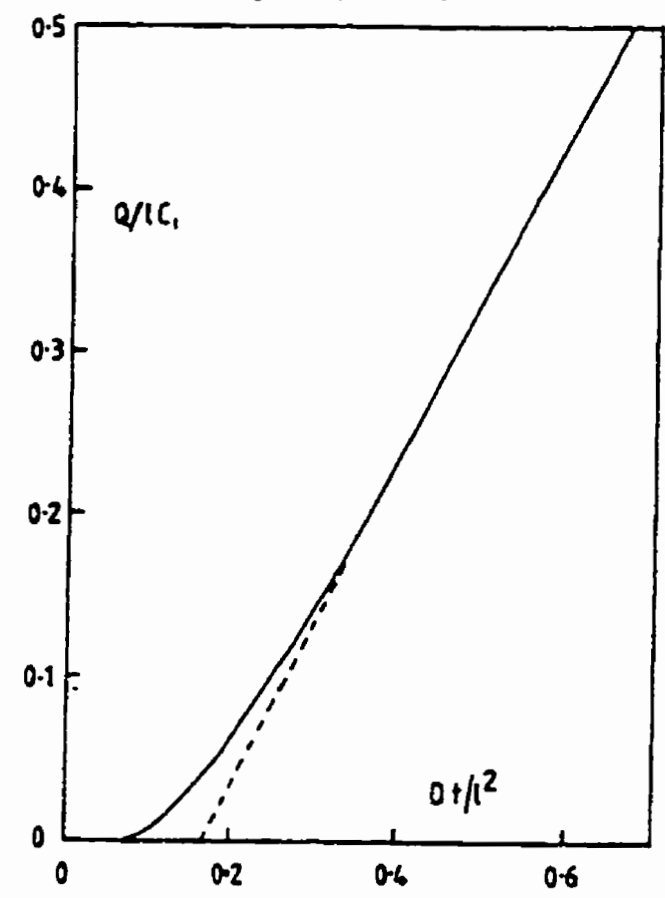

Permeation through a film approaching the steady state.

Figure 15: Concentration Profile of Membrane with Aqueous Boundary Layers

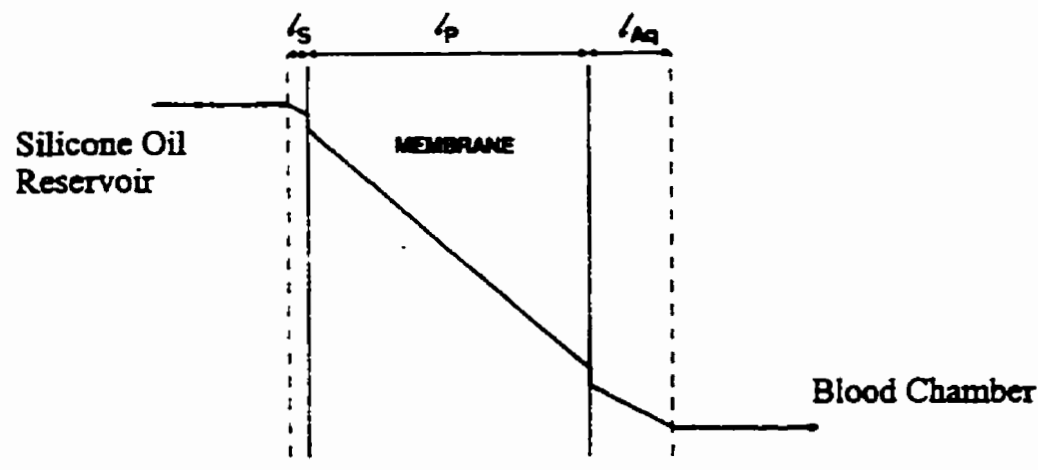


Figure 16: Absorption Plot: Gent et al, (1982)

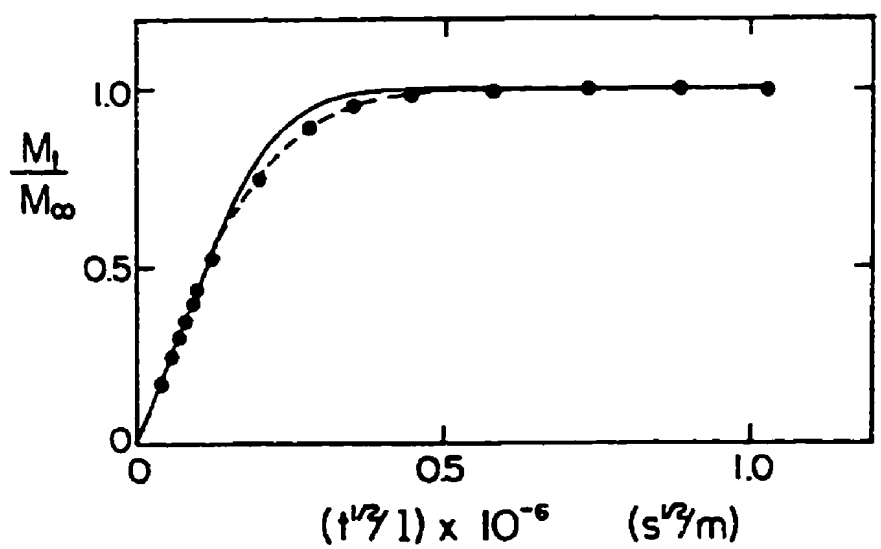

Weight uptake $m_{l}$ of PDMS liquid $\left(\bar{M}_{n}=4,700\right)$, relative to the equilibrium value $m_{\text {.. }}$ vs. $t^{1 / 2} / l$. where $t$ is the time of immersion in the swelling liquid and $l$ is the thickness of the sample. a thin sheet of an endlinked PDMS network having $M_{c}=11,500$. Full curve calculated from eq. (2).

Figure 17: Permeation and Lag Time Curve: Comyn, (1985)

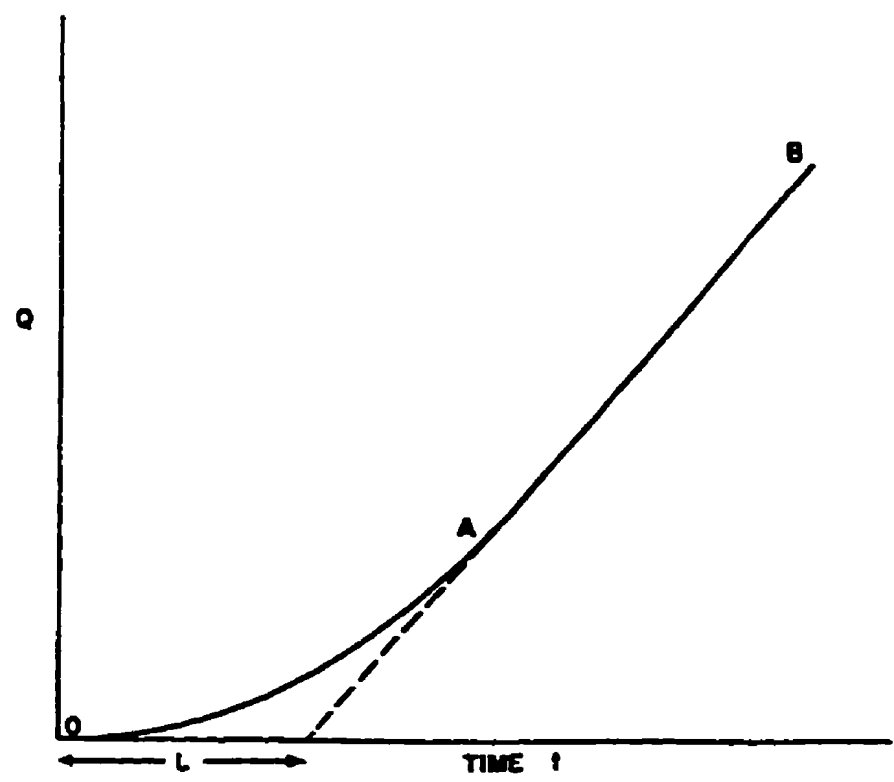

Typical permeation and time lag curve. Amount of permeated penetrant, $Q$, as a function of time, 2 Extrapolation of the steady state line $A B$ to the time axis gives the time lag as intercept $L$ 
Figure 18: Assembled Testing Tube

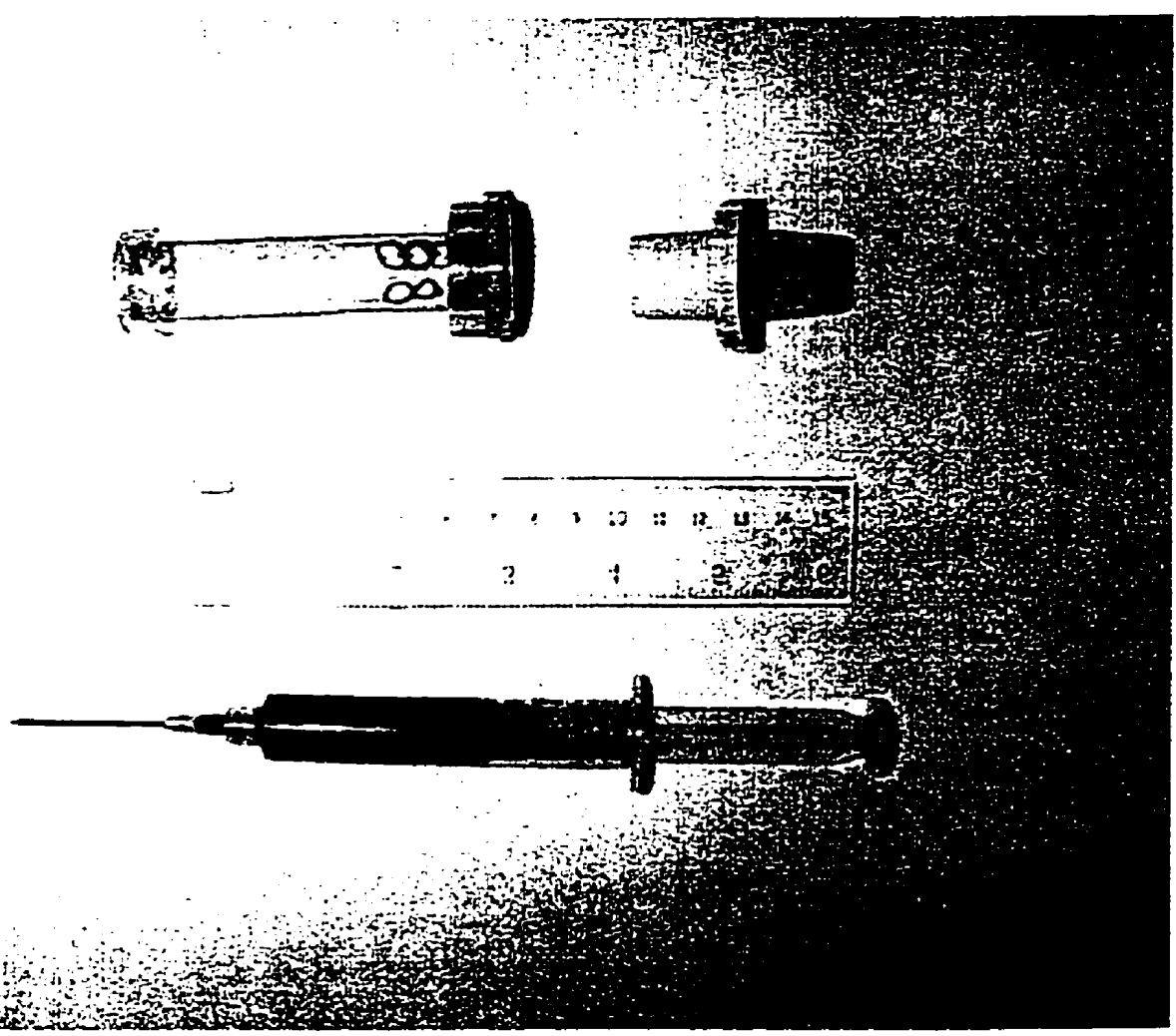

Figure 19: Solvent Sample After Extraction

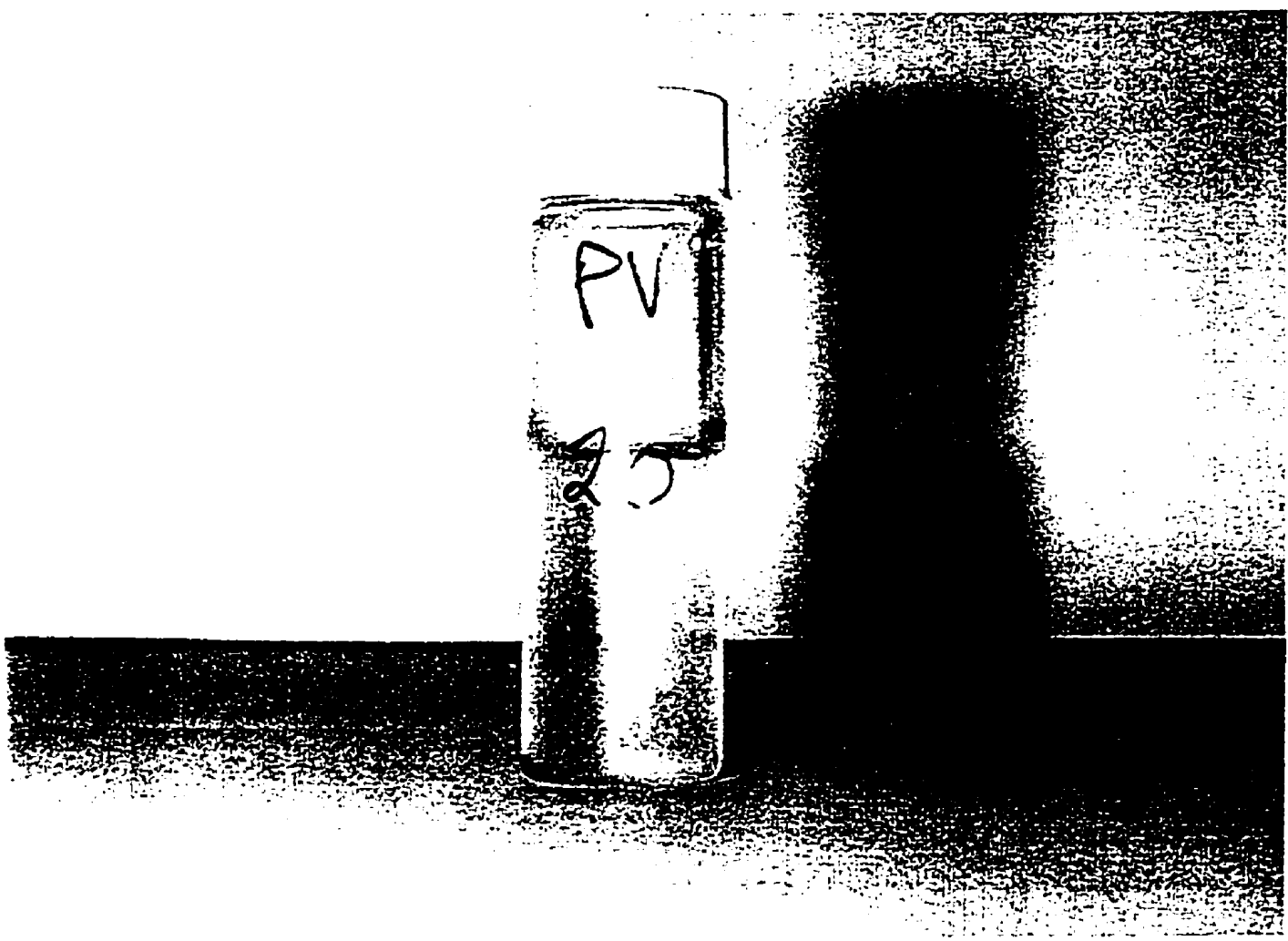


Figure 20: Injection Syringe and $1.5 \mathrm{ml}$ Vial

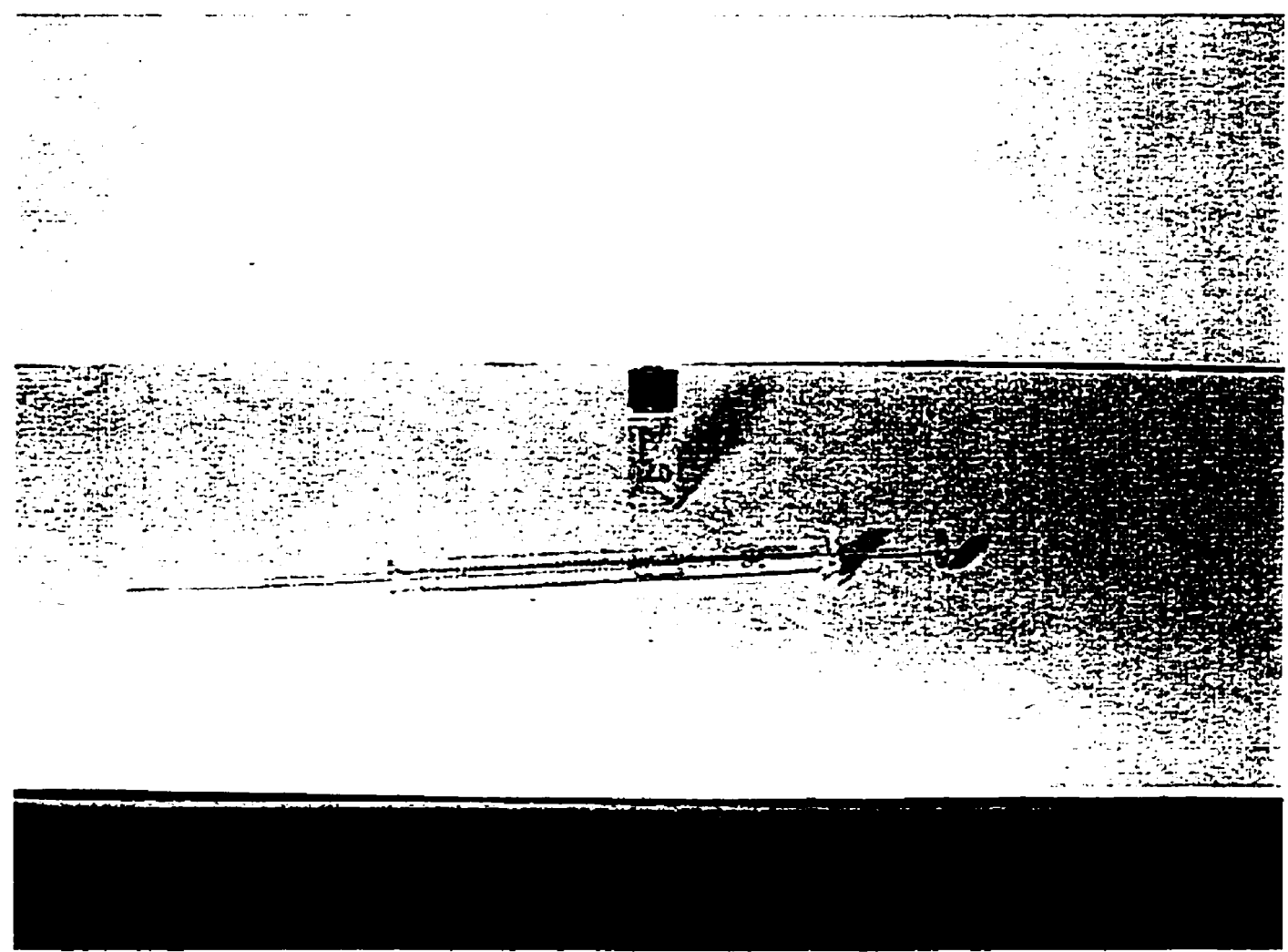

Figure 21: Blood Bag and $10 \mathrm{cc}$ Syringe Used to Remove Blood

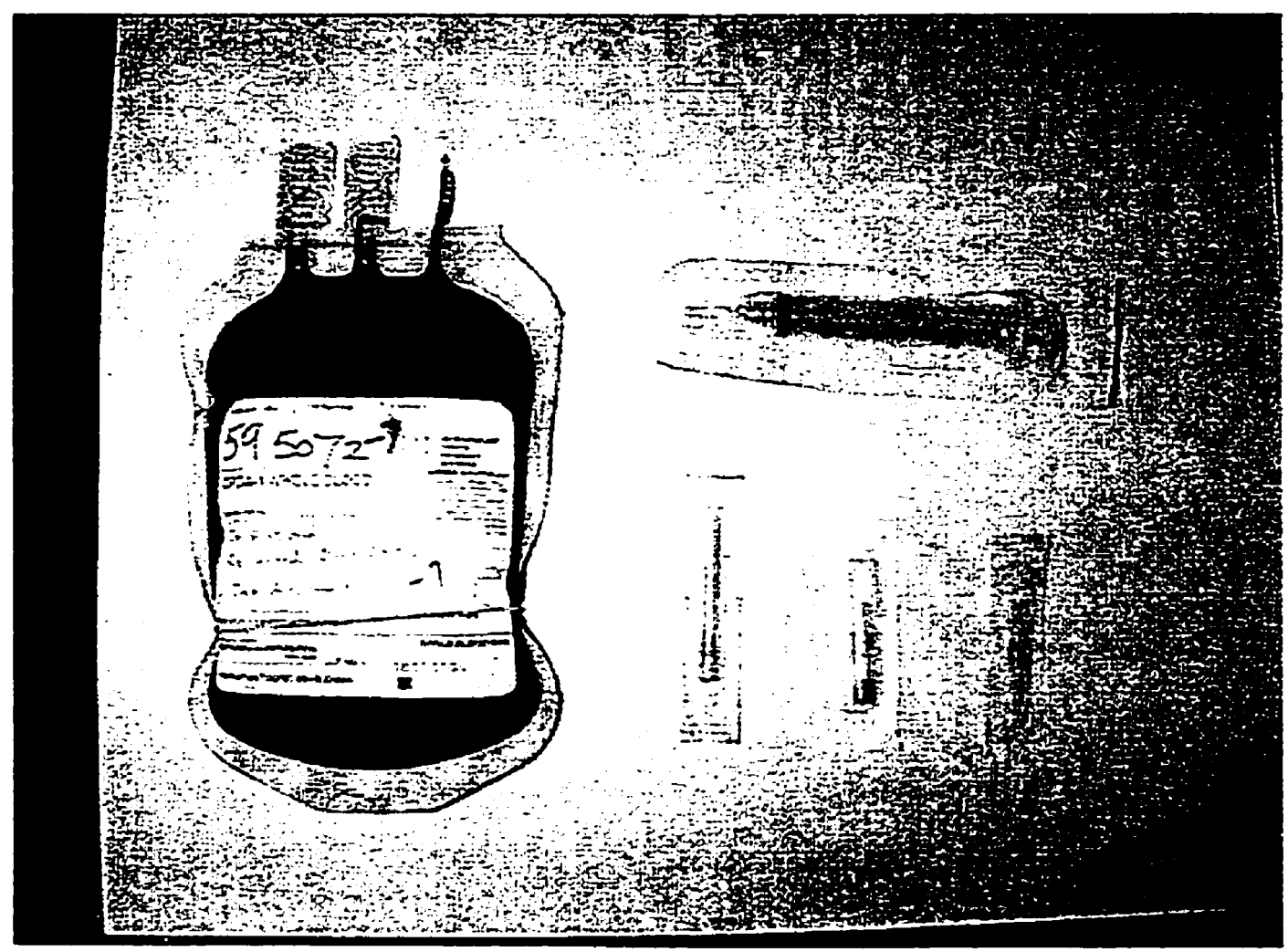


Figure 22: Test Apparatus in Incubator on Stirring Tray

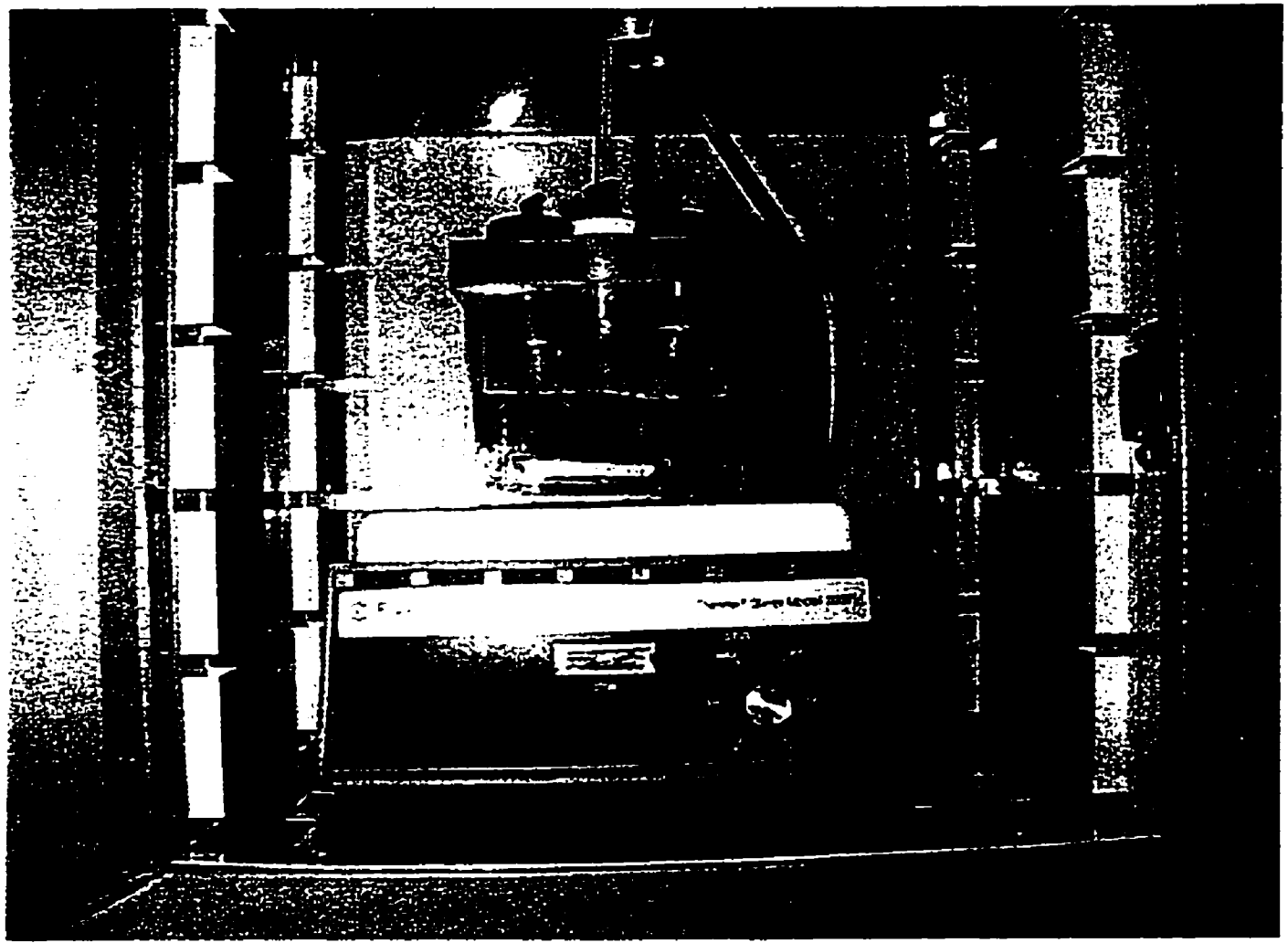

Figure 23: Extraction Turn Table

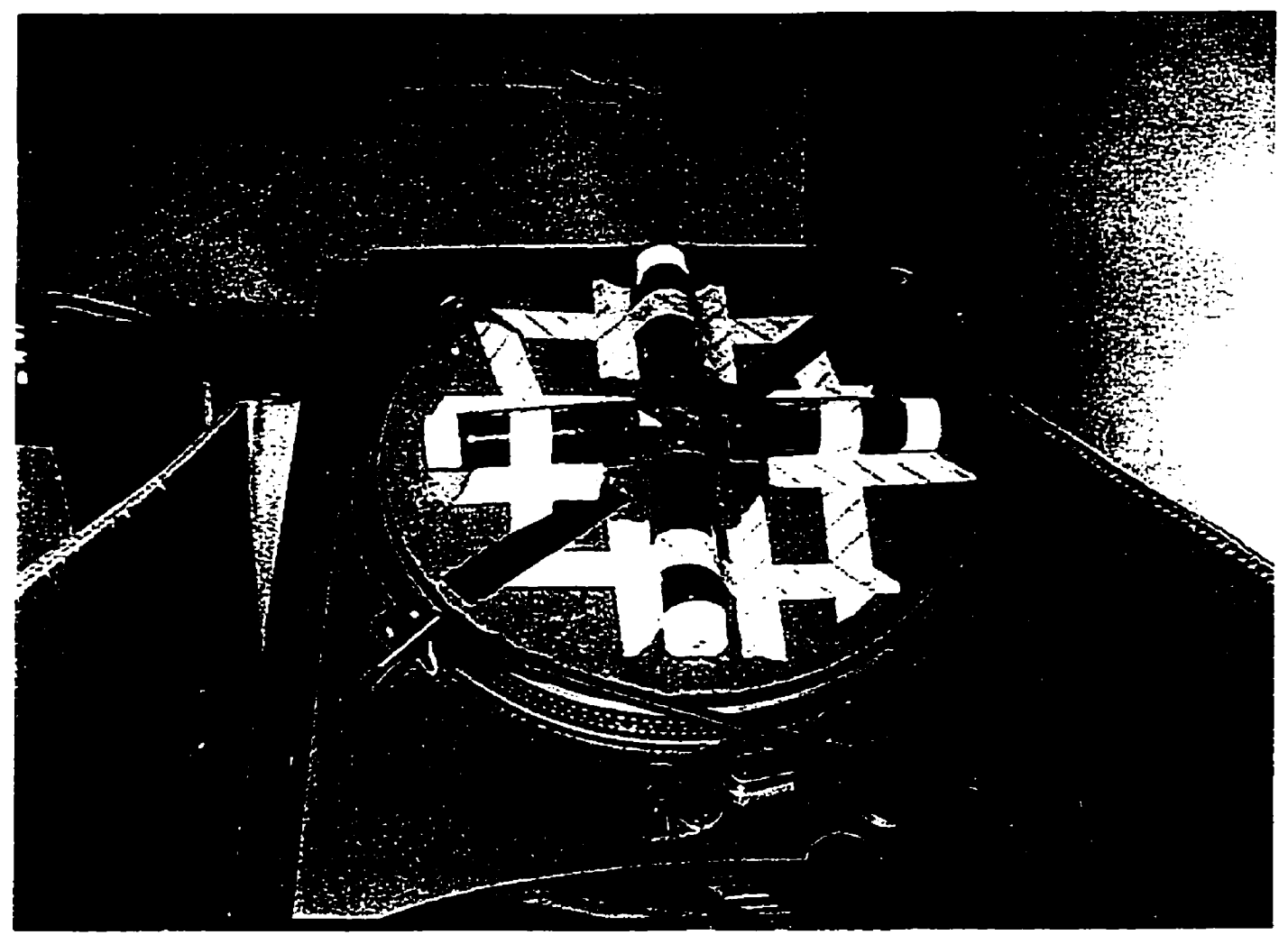


Figure 24: Unassembled Testing Tube

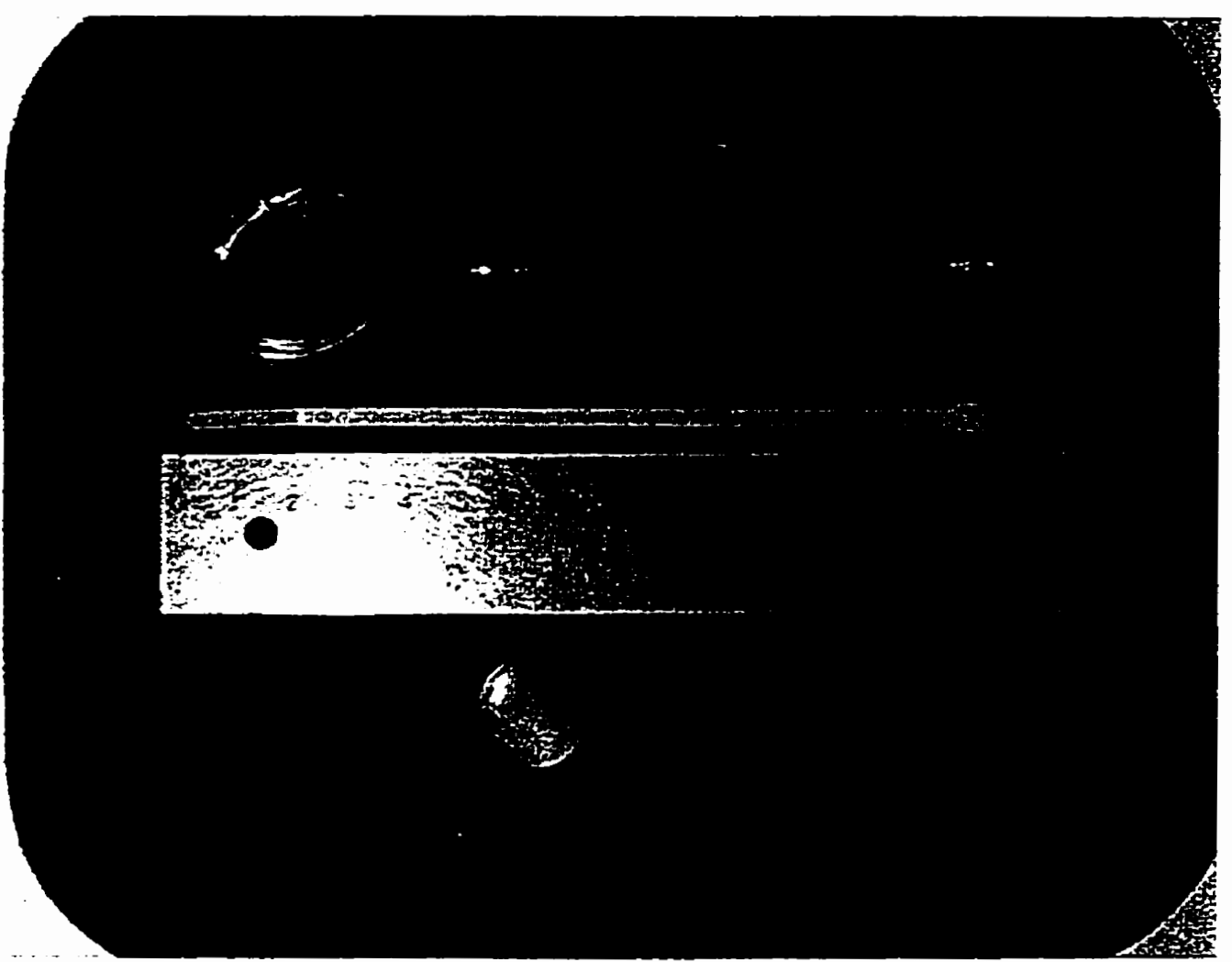

Figure 25: Magnetic Stir Bar in Silicone Oil Reservoir

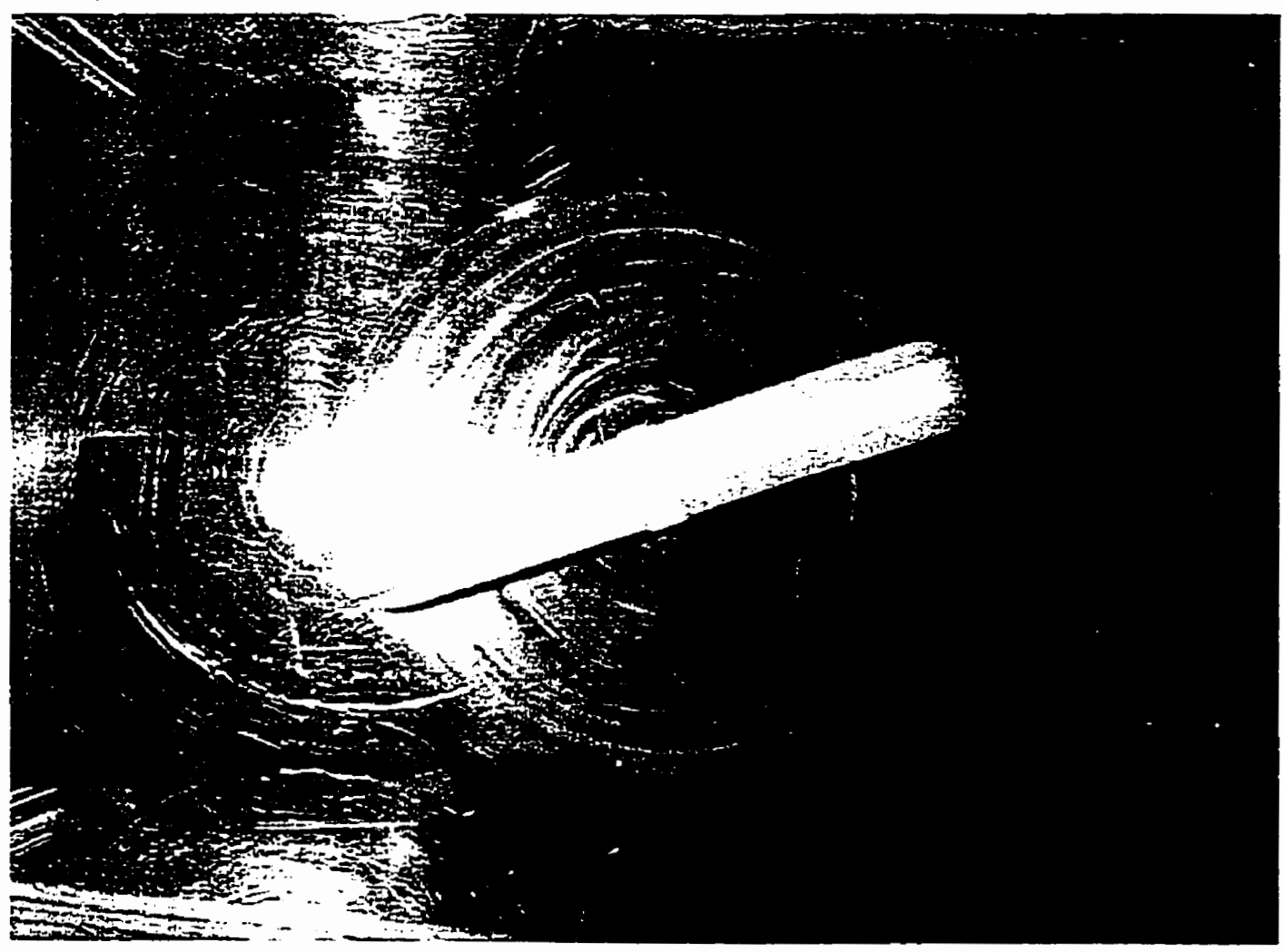


Figure 26: Gravimetric Absorption Test - Biospan Sheet in Silicon Oil

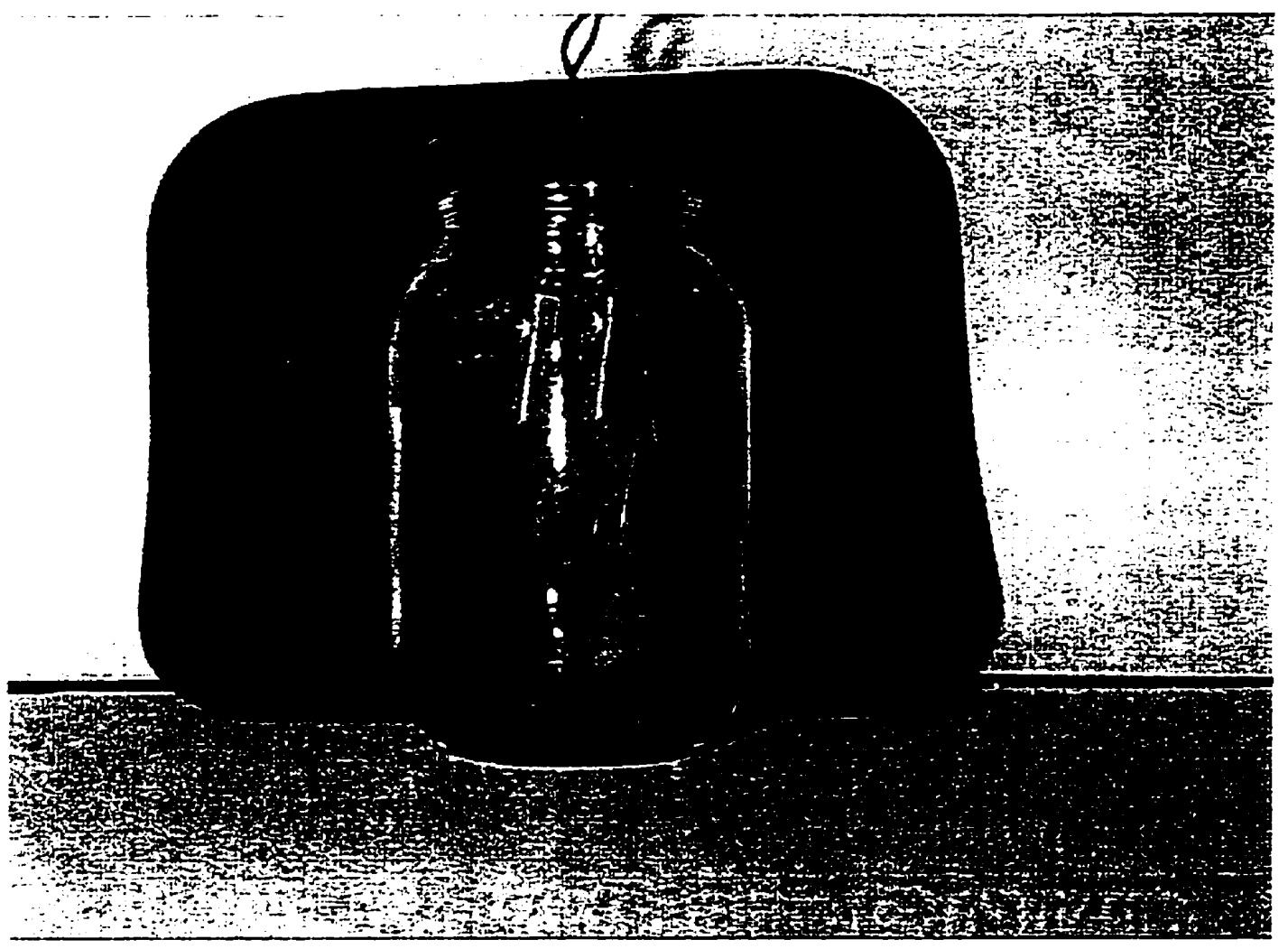

Figure 27: Biospan Sheets Suspended on Clips

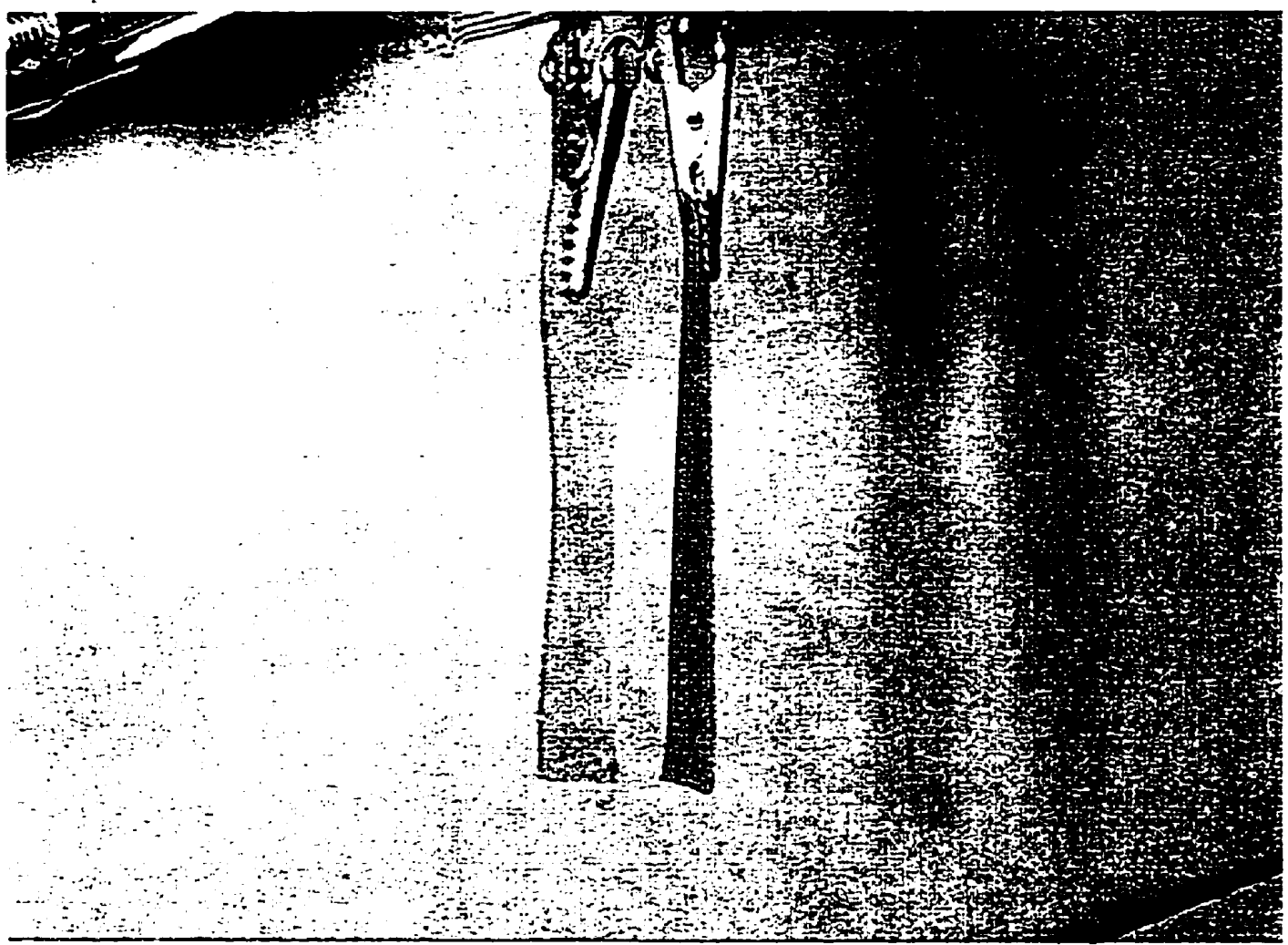


Figure 28: Permeability Test End-Caps

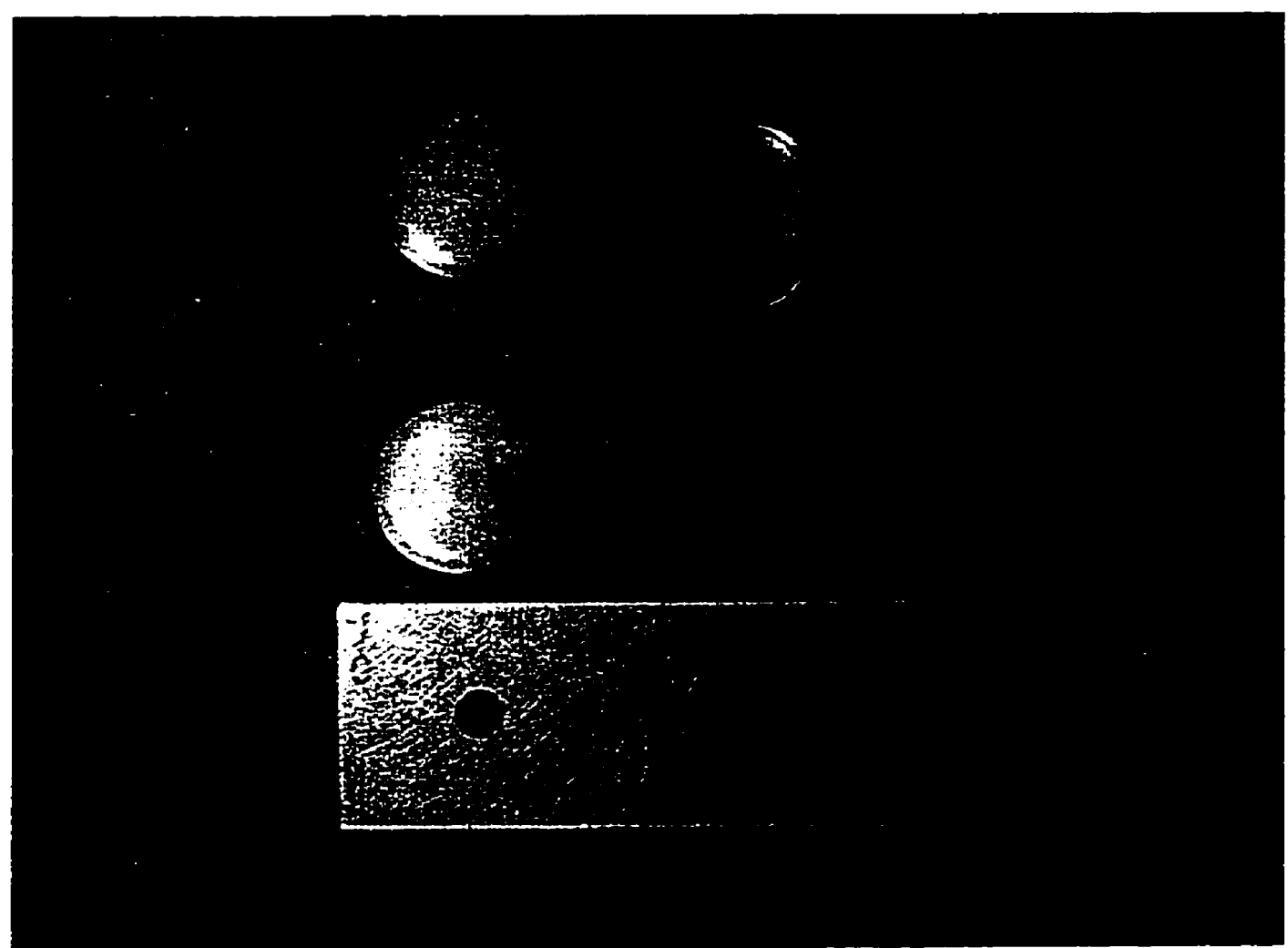

Figure 29: Biospan Sheet - Absorption Test GC/MS Sheet Test

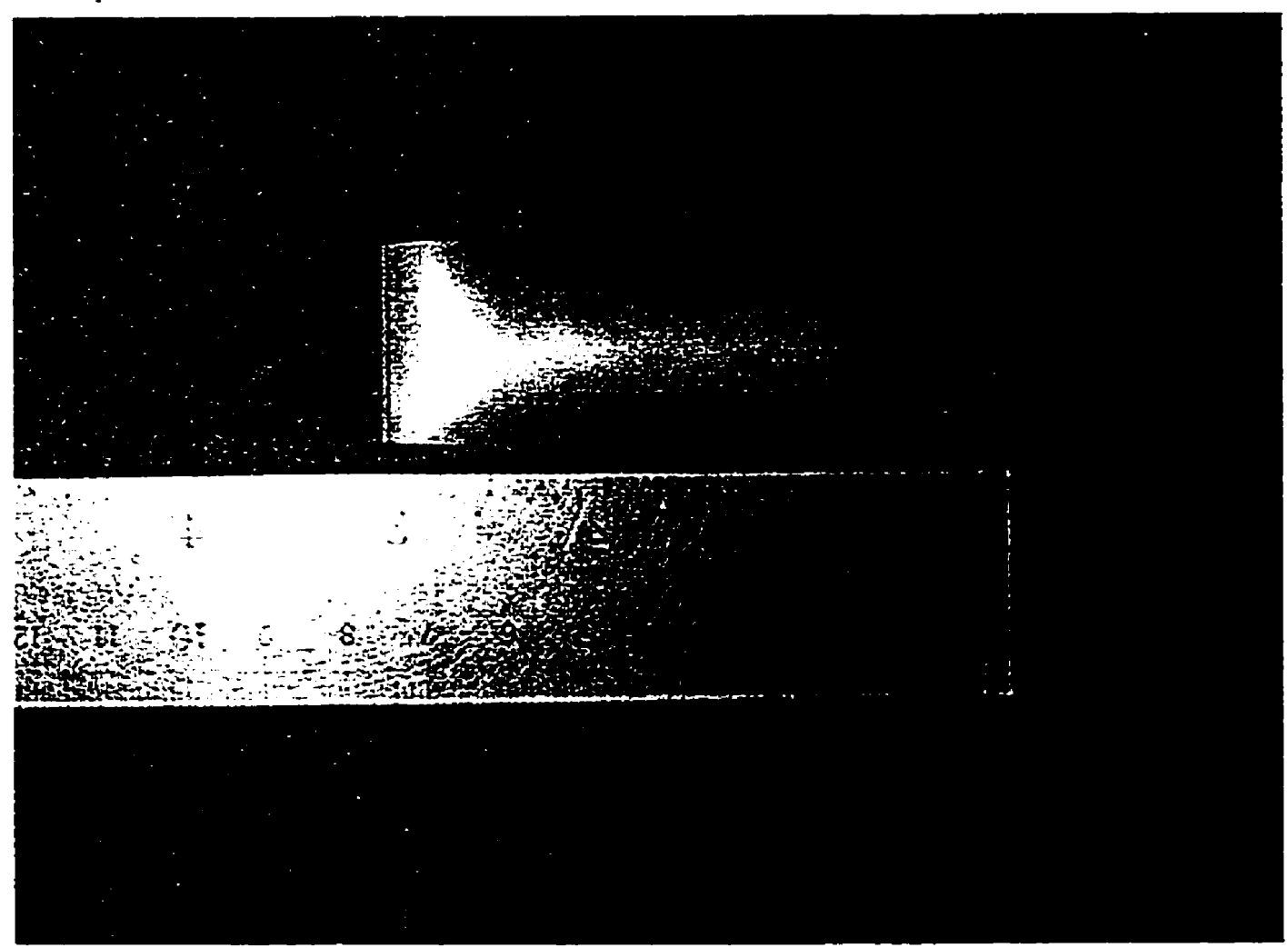


Figure 30: Fluid Biospan in $60 \mathrm{cc}$ Syringe

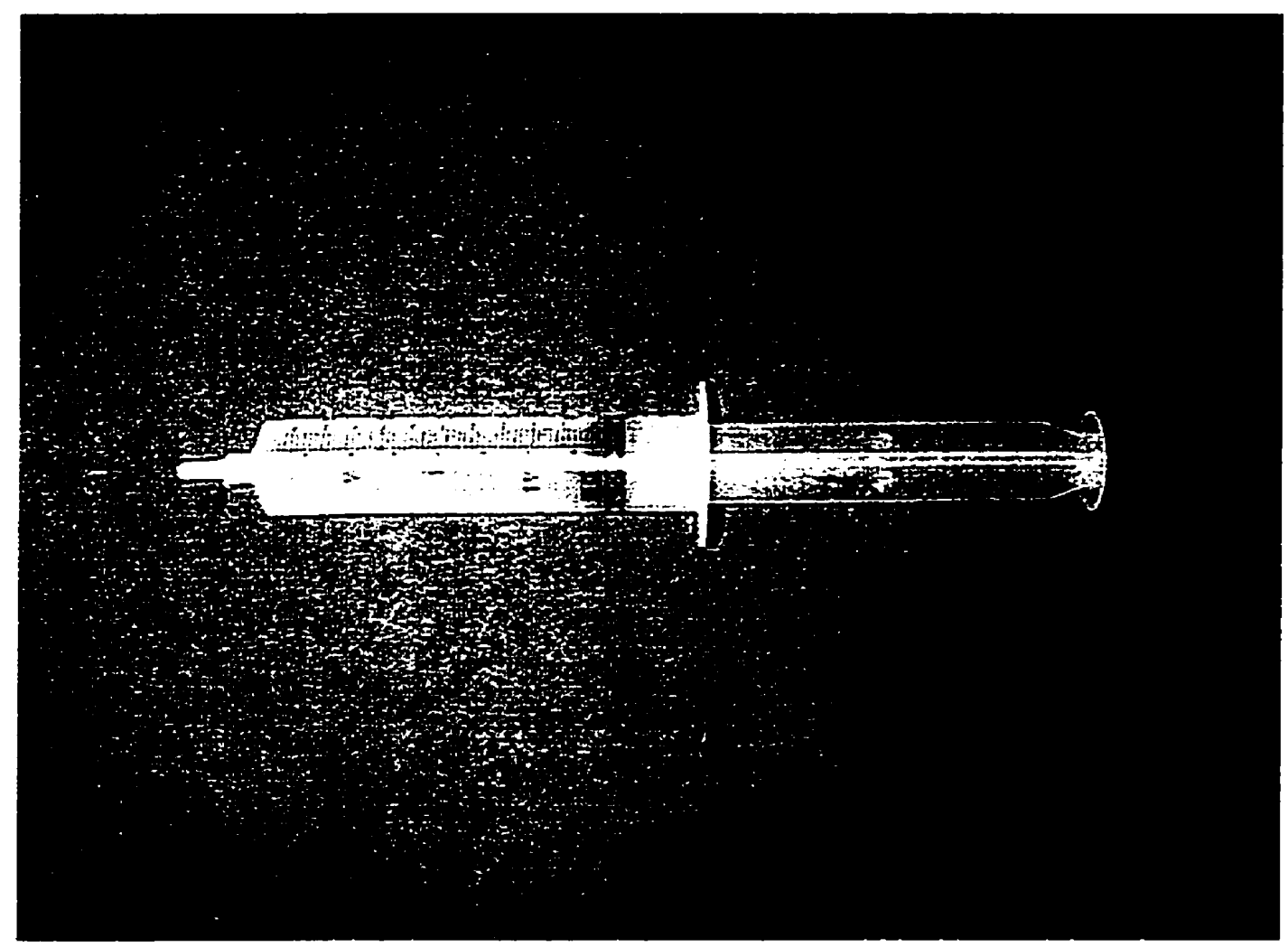




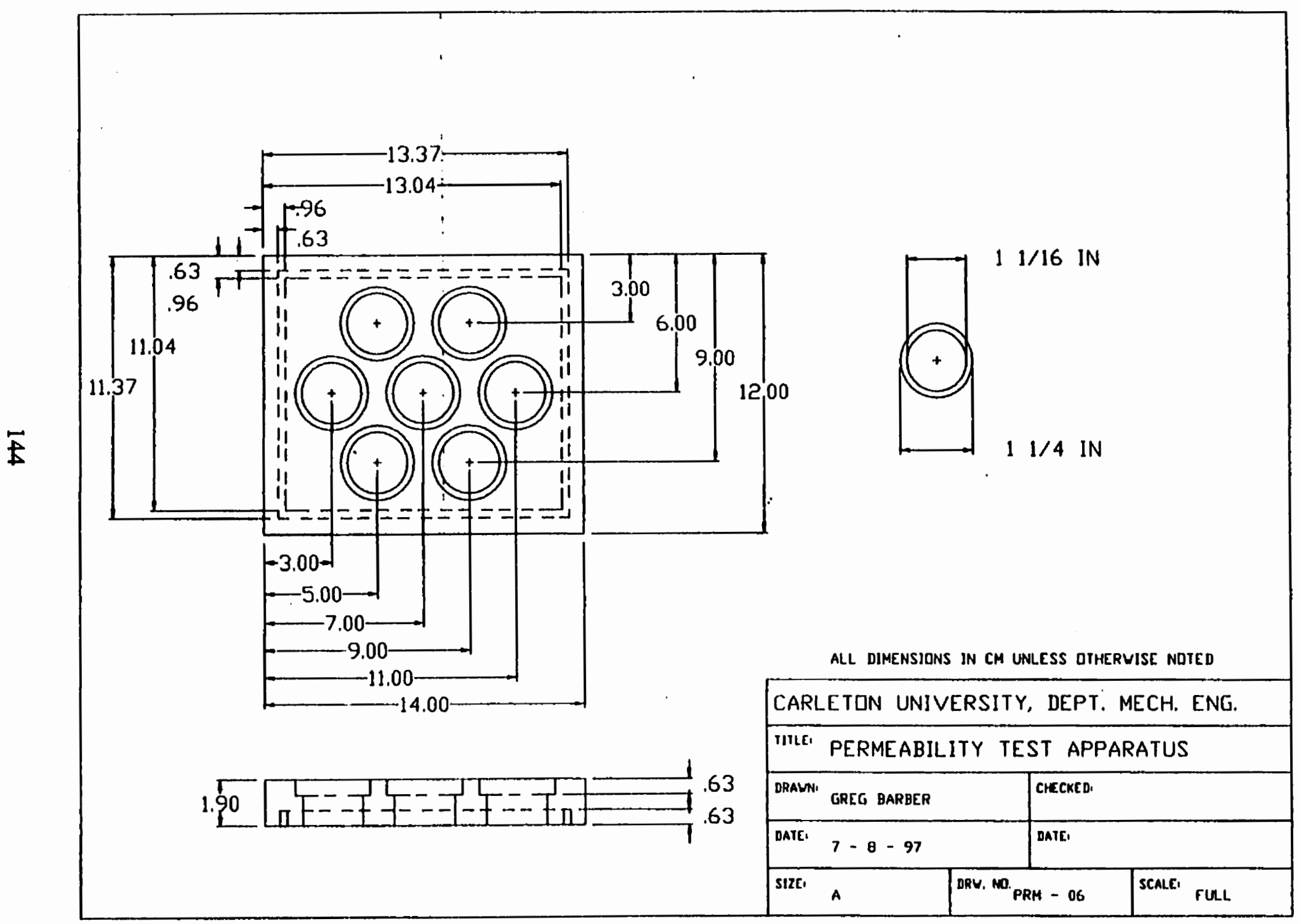

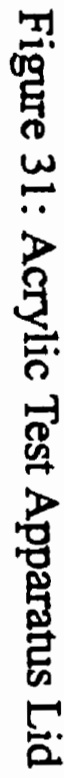




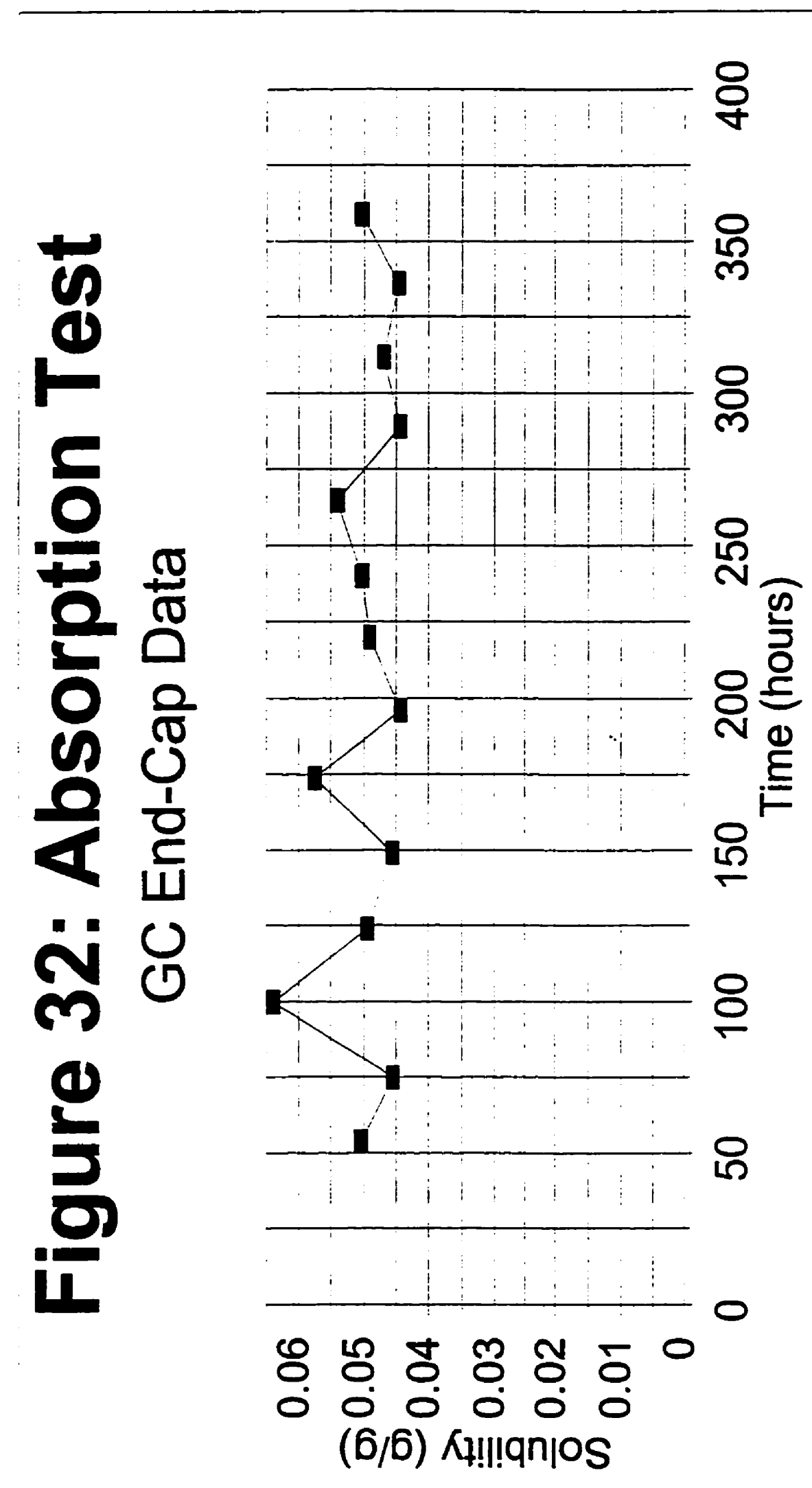




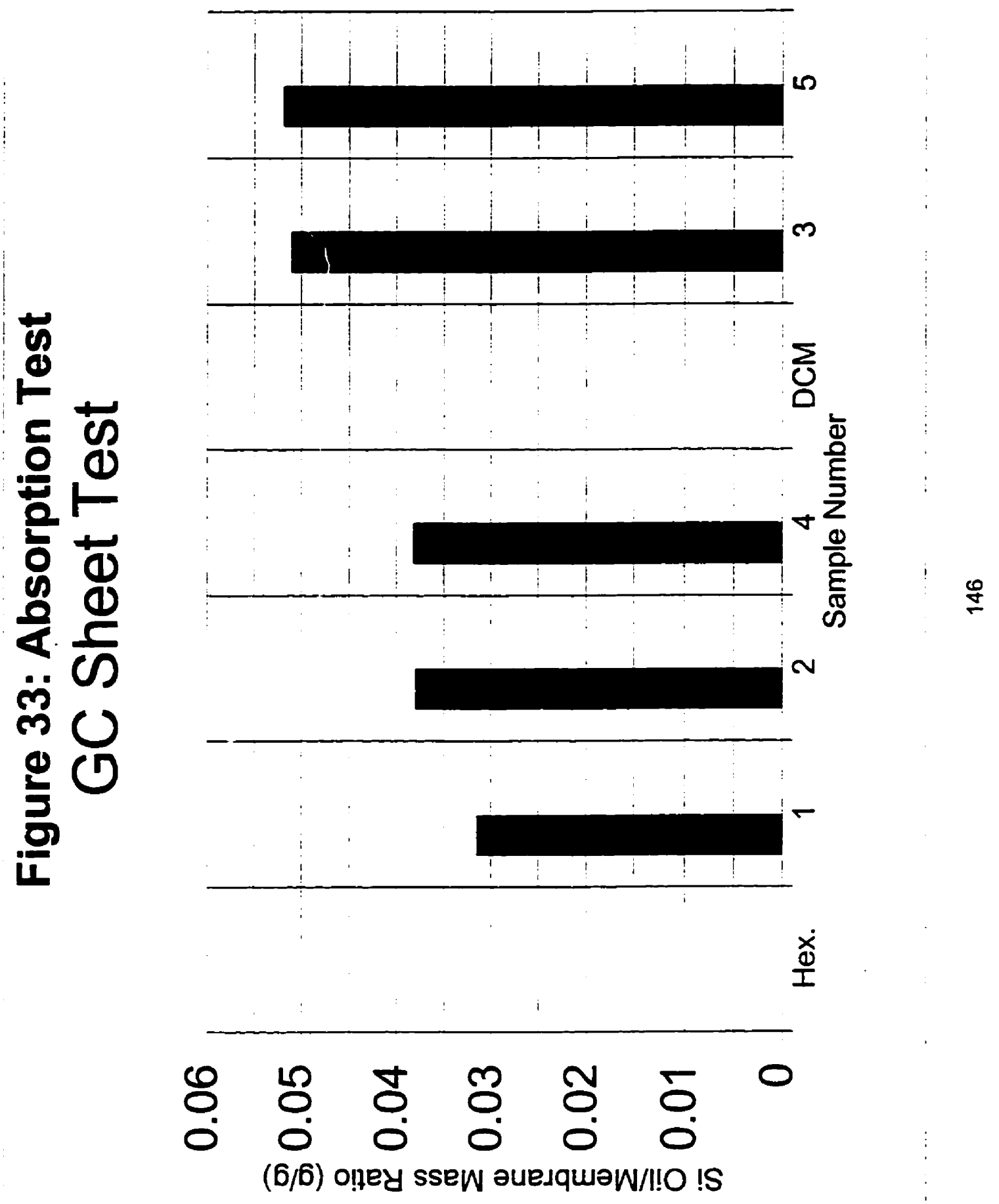




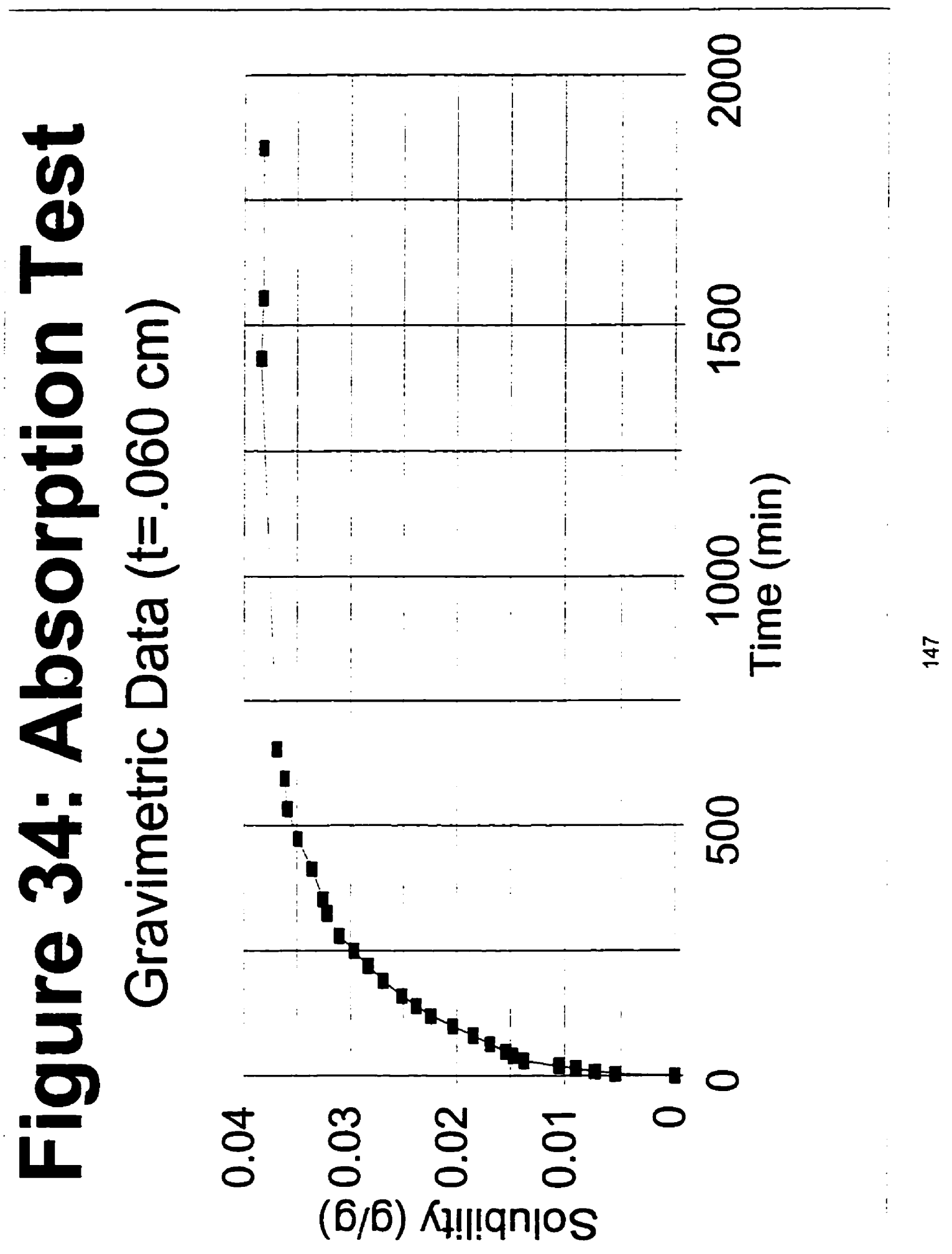




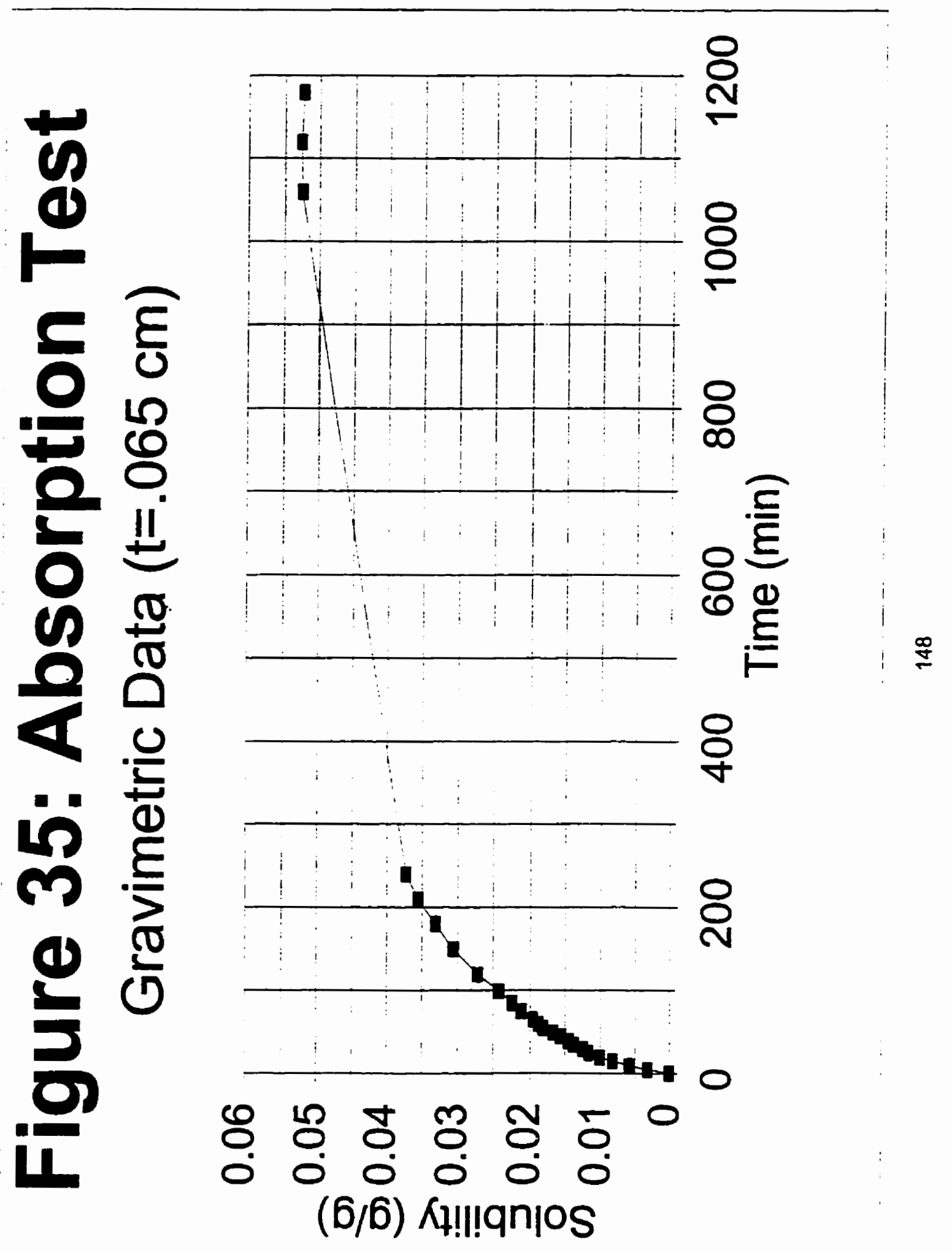




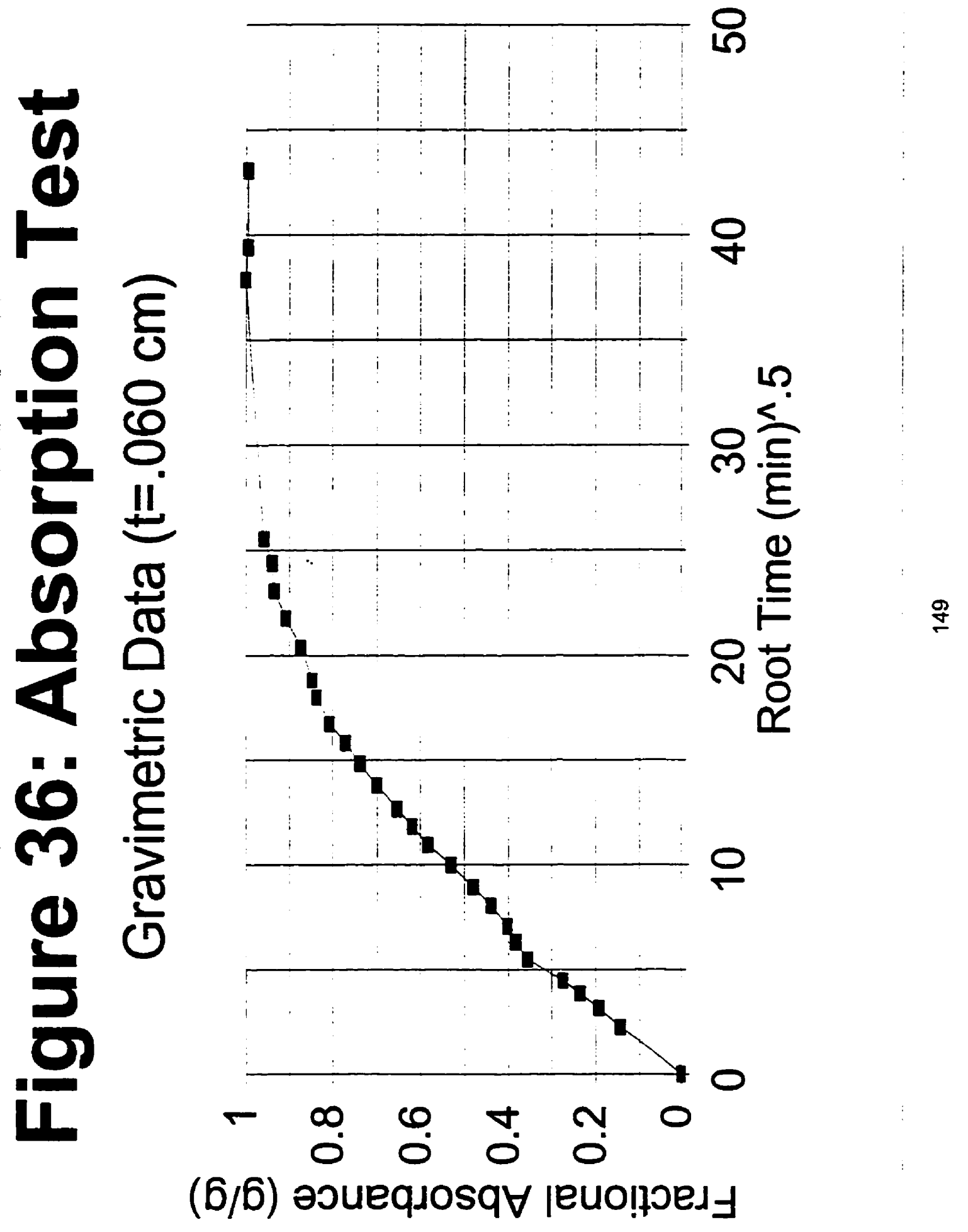




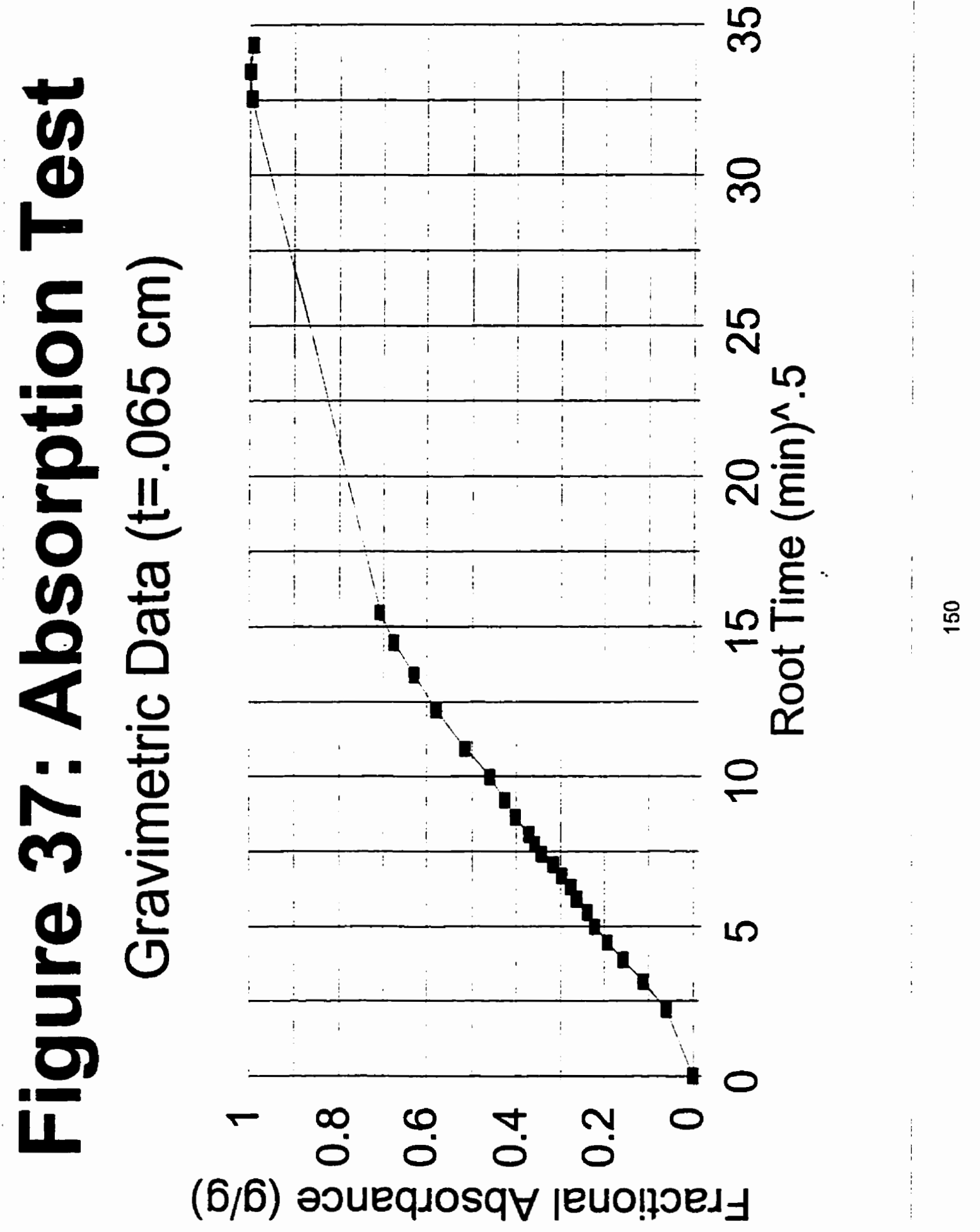




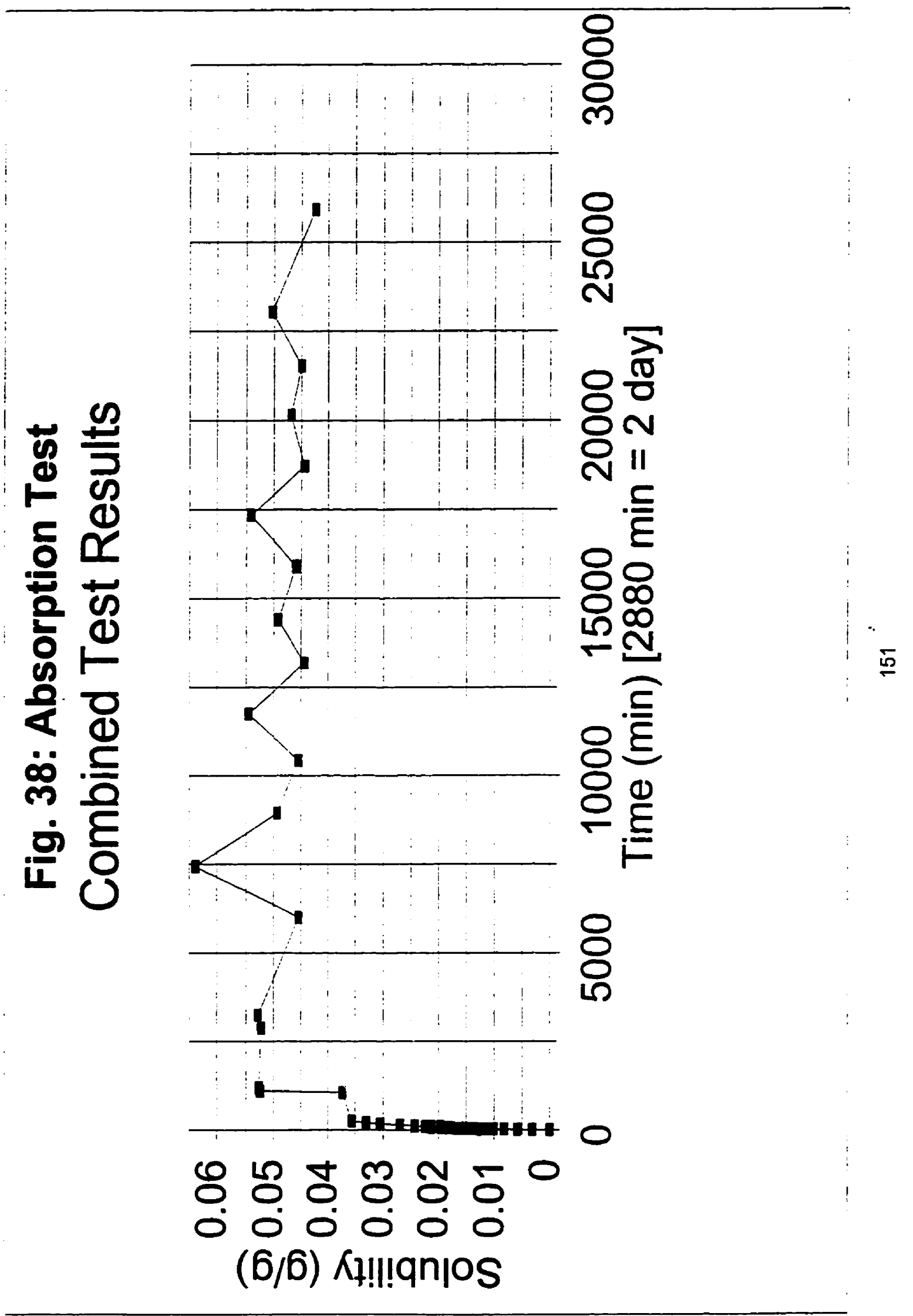




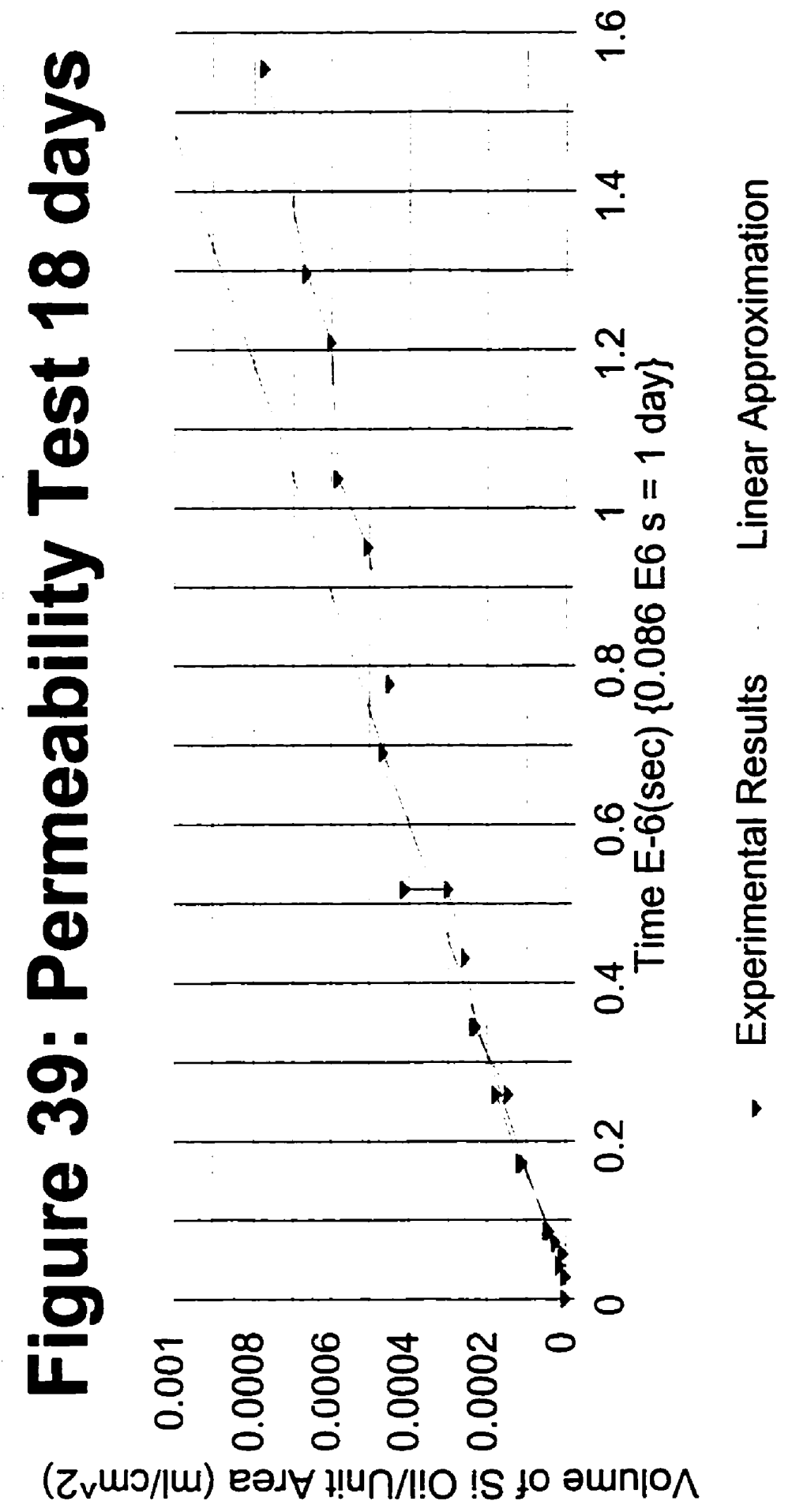

ชิ 


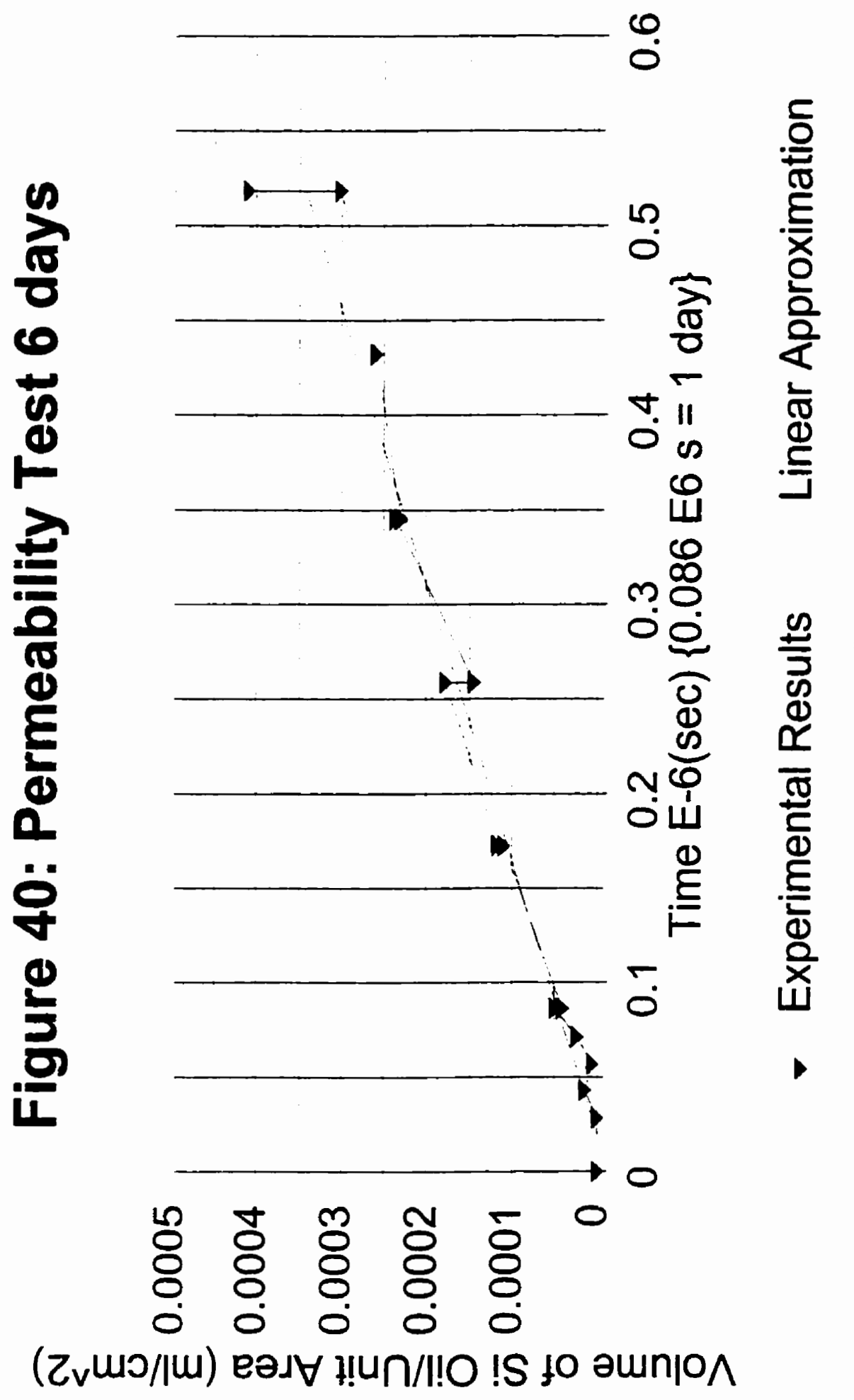


Figure 41: Comparison of Permeability Test Blood Before and After 6 Days

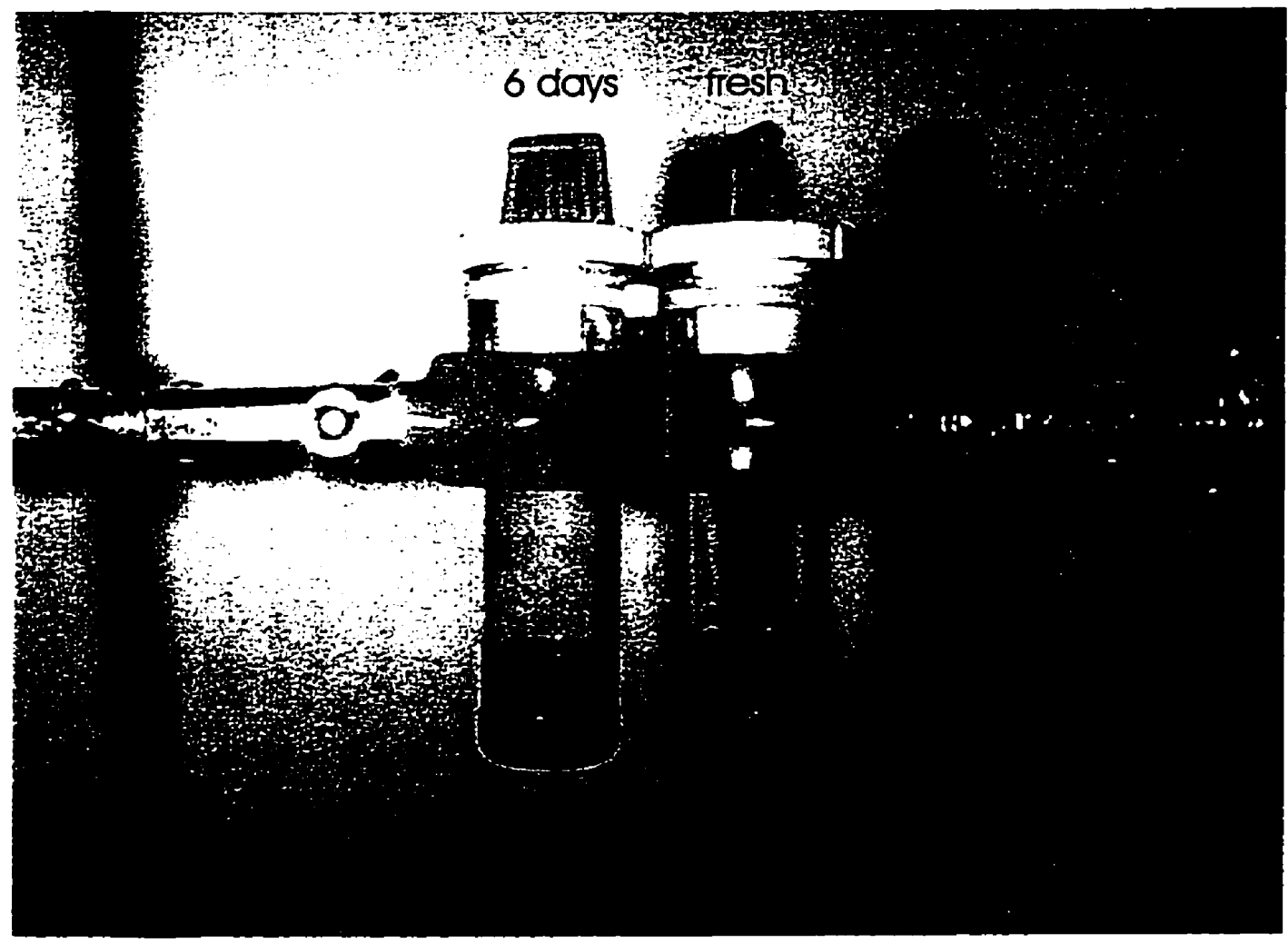

Figure 42: Mixed Solvents DCM and Hexane with Blood

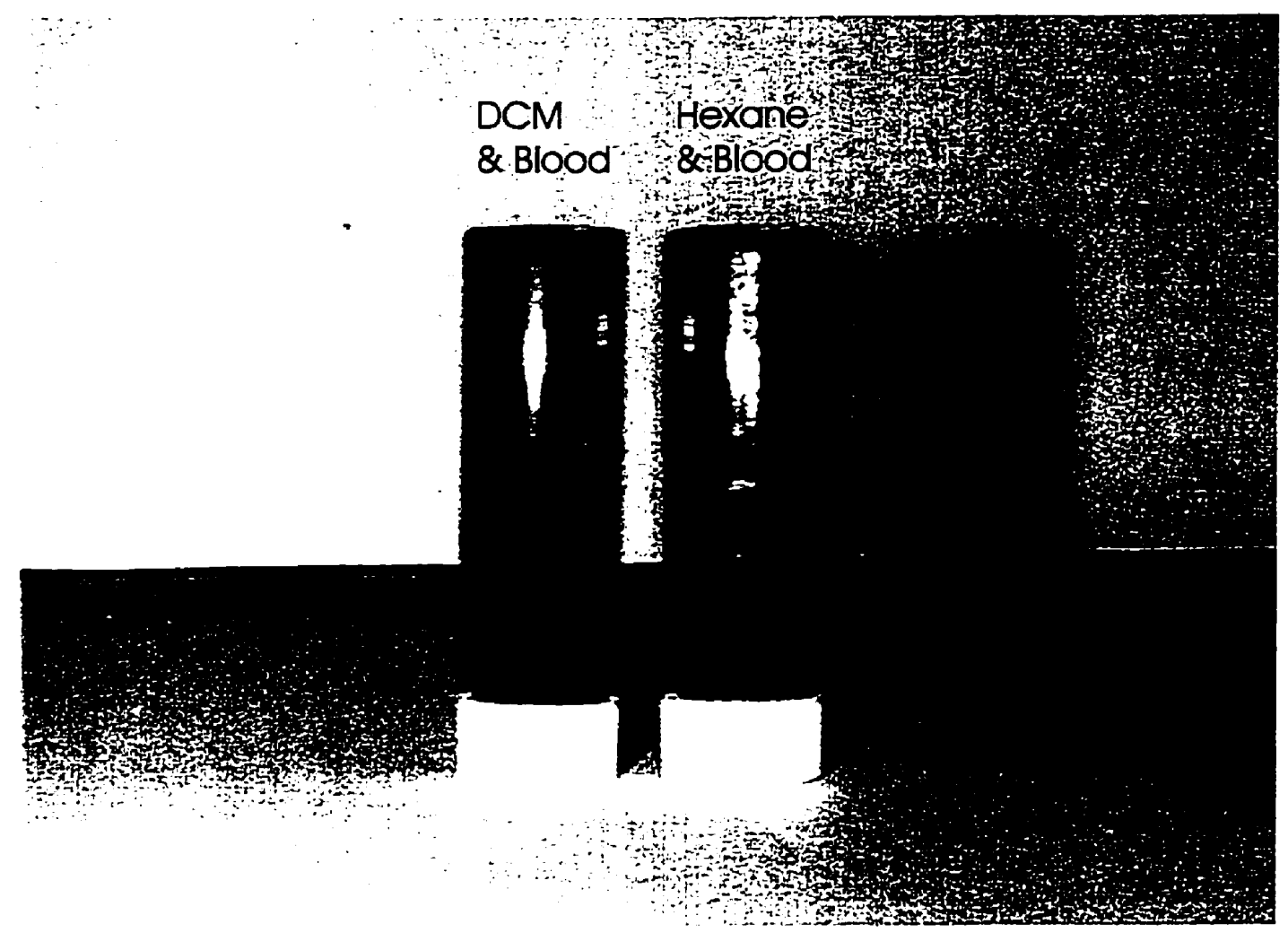


Figure 43: Centrifuged Hexane and Blood Sample

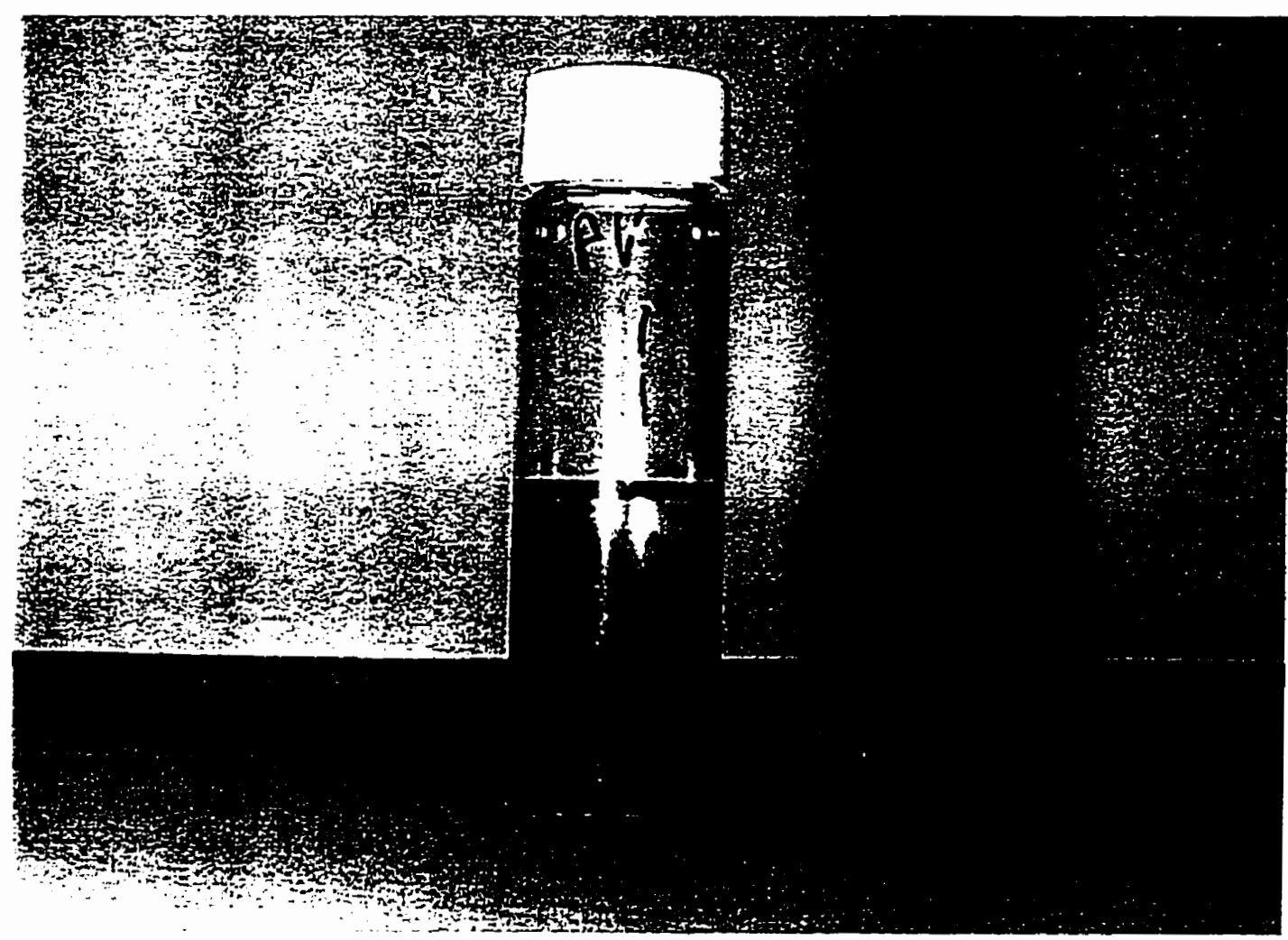

Figure 44: Mixed Solvents DCM and Hexane with Blood

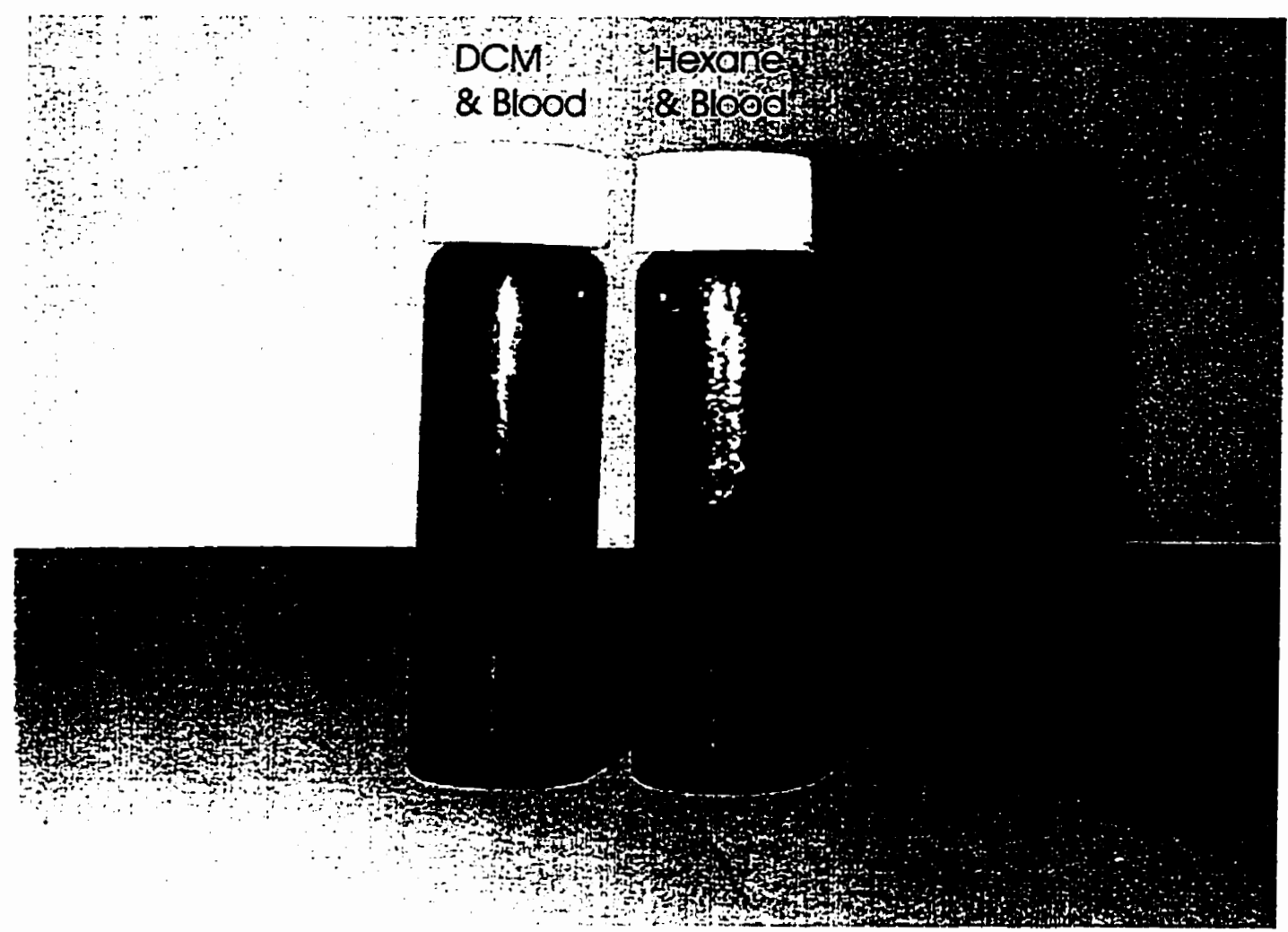


Figure 45: Centrifuged DCM and Blood Samples

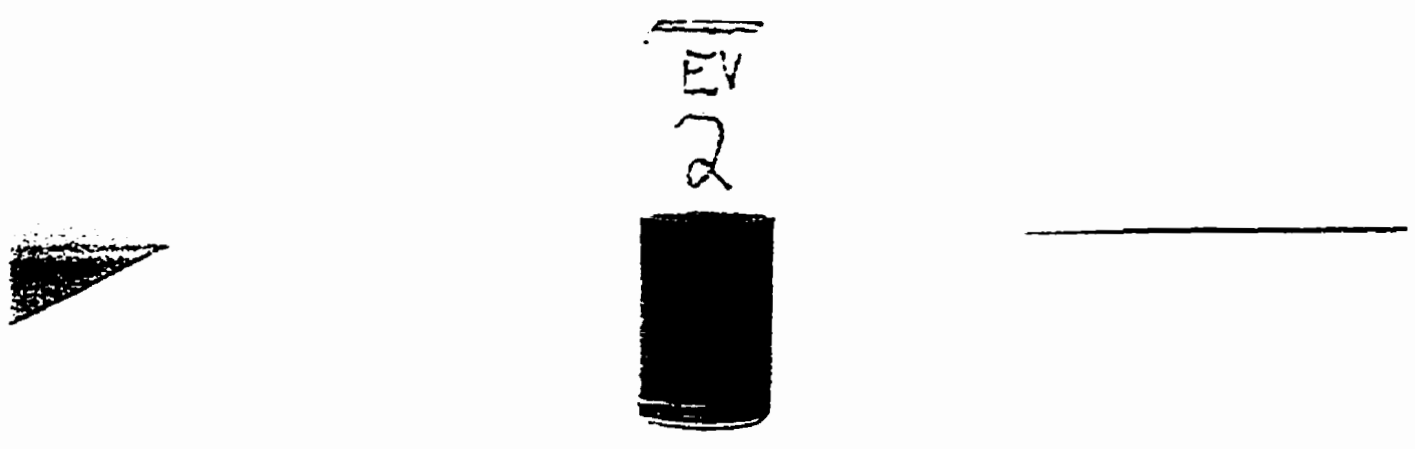




\section{APPENDIX C:}

Tables 
Table 1: Comparison of Human and Bovine Blood

\begin{tabular}{|c|c|c|c|c|c|}
\hline & & $\begin{array}{l}\mathrm{RBC} \\
\mathrm{XE}-12 \pi\end{array}$ & $\begin{array}{l}\text { WBC } \\
\times E-9 / 1\end{array}$ & $\begin{array}{l}\text { Hemoglobin } \\
\text { g/dl }\end{array}$ & $\begin{array}{l}\text { Platelet } \\
\text { xE-9n }\end{array}$ \\
\hline Human & Male & 5.2 & 7.8 & 15.7 & 311 \\
\hline Human & Female & 4.6 & 7.8 & 13.8 & 311 \\
\hline Bovine & & 7.5 & 8 & 11.5 & 450 \\
\hline
\end{tabular}

Table 2: Biocompatible Elastomers

\begin{tabular}{|l|l|}
\hline Biomaterial & Molecular Structure \\
\hline Biomer & polyether polyurethane \\
\hline Biospan* & polyether polyurethane \\
\hline Biospan -S & polyether polyurethane with polydimethylsiloxane surface \\
\hline Biospan - SC & polycarbonate polyurethane with polydimethylsiloxane surface \\
\hline Biolon & polyether polyurethane \\
\hline ChronoFlex AR & polycarbonate polyurethane \\
\hline ChronoThane B* & polyether polyurethane \\
\hline Hexsyn & polyhexene rubber \\
\hline Santoprene & polypropylene elastomer \\
\hline Avcothane & urethane/silicone copolymer \\
\hline Chemigum & nitrile rubber \\
\hline Pellethane & polyether urethane \\
\hline Novithane & polyurethane \\
\hline Butyl rubber & butyl rubber \\
\hline Pursten & natural rubber \\
\hline Silicone rubber & poly(dimethylsiloxane) \\
\hline Angioflex & unknown \\
\hline
\end{tabular}

- denotes Biomer clones

Table 3: Biospan Material Characteristics

Typical Property (dry)

The Polymer Technology Group, Incorporated Product Literature

\begin{tabular}{|l|l|l|}
\cline { 2 - 3 } \multicolumn{1}{c|}{} & $\begin{array}{l}\text { ASTM } \\
\text { Standard }\end{array}$ & $\begin{array}{l}\text { ASTM } \\
\text { Value }\end{array}$ \\
\hline Initial Modulus & $\mathrm{D}-1708$ & $900 \mathrm{psi}$ \\
\hline Tensile Strength & $\mathrm{D}-1708$ & $6550 \mathrm{psi}$ \\
\hline Ultimate Elongation & $\mathrm{D}-1708$ & $815 \%$ \\
\hline Hardness & $\mathrm{D}-2240$ & $75 \mathrm{~A}$ \\
\hline Glass Transition Temp. & $\mathrm{D}-3418$ & $-650 \mathrm{C}$ \\
\hline Water Absorption & $\mathrm{D}-570$ & 0.015 \\
\hline Weight Average MW & $\mathrm{D}-3593$ & 186,000 daltons \\
\hline Color & NA & Translucent \\
\hline Solution Concentration & NA & $<25 \%$ (wt/wt) \\
\hline
\end{tabular}


Table 4: Test Evaluation Results

\begin{tabular}{|l|l|l|r|}
\cline { 2 - 4 } \multicolumn{1}{c|}{} & $\begin{array}{l}\text { Concentration } \\
\text { PPM } \\
\text { (Known) }\end{array}$ & $\begin{array}{l}\text { Concentration } \\
\text { PPM } \\
\text { (Found experimentally) }\end{array}$ & \% error \\
\hline sample 1 & 53 & 58 & 9.4 \\
\hline sample 2 & 33 & 36 & 9.1 \\
\hline
\end{tabular}



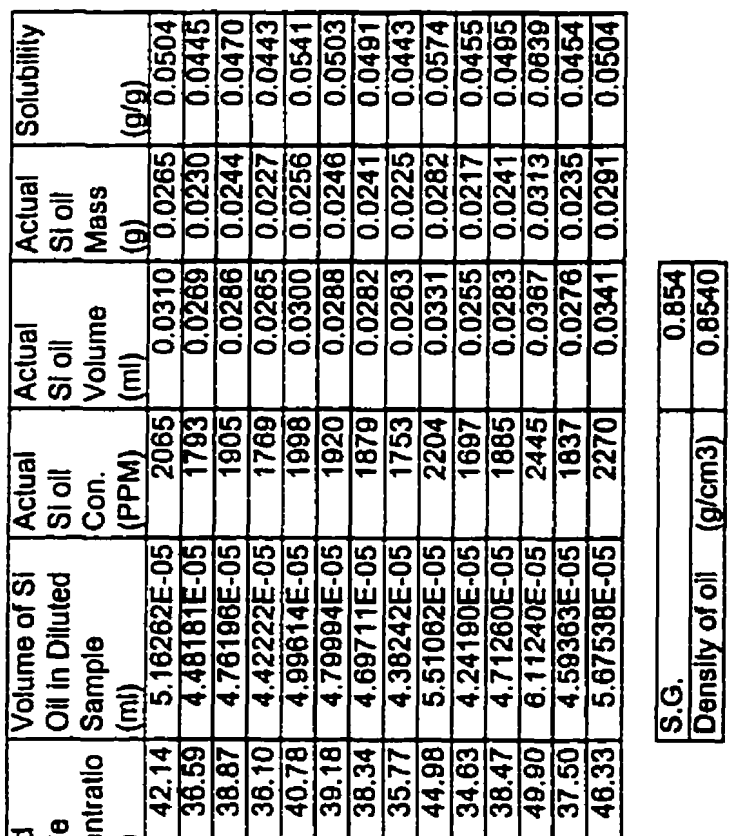
옥 을 웜

言㤐 80

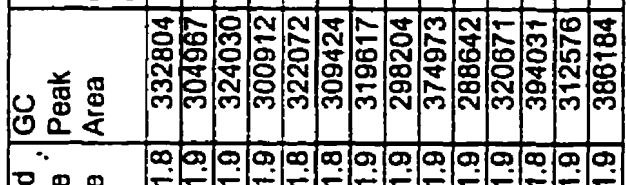

Do $\div \div=\because \div$ 总总言

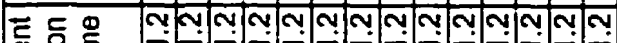
怘棓言
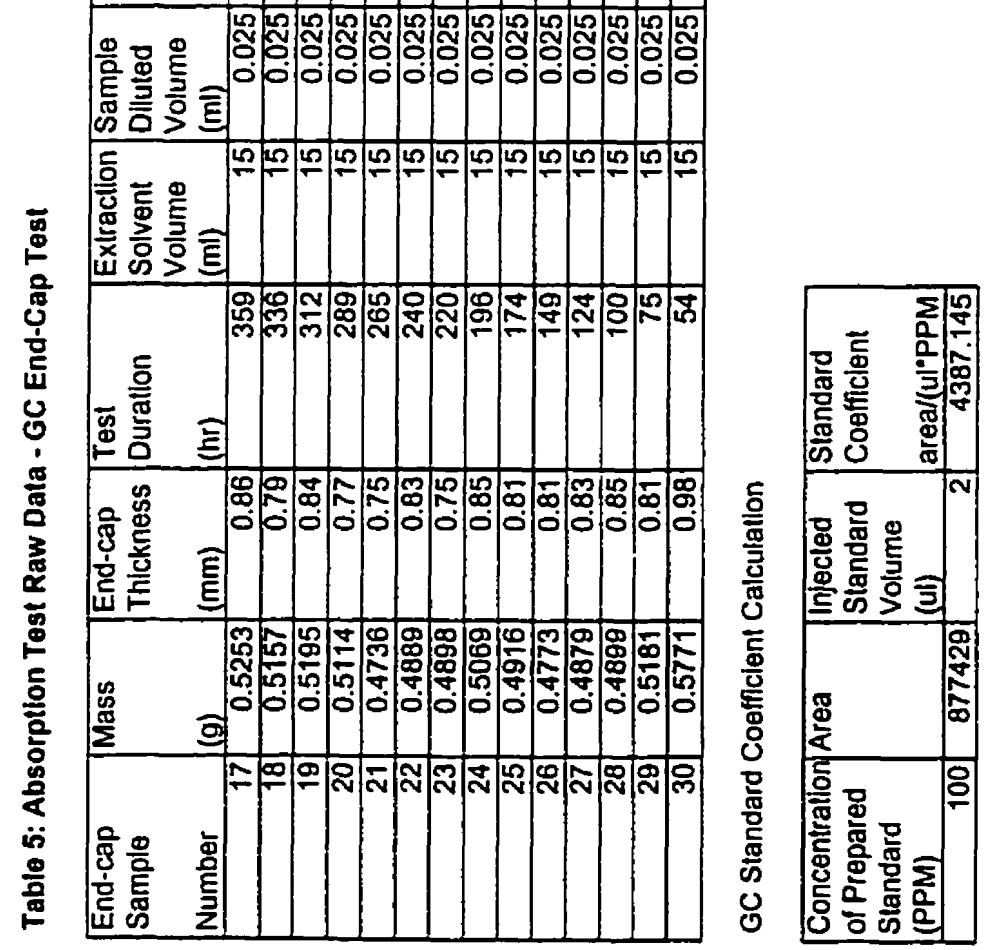
Table 6: Absorption Test Raw Data - GC Sheet Test

\begin{tabular}{|c|c|c|c|c|c|c|c|c|c|c|c|c|c|c|}
\hline $\begin{array}{l}\text { Samplo } \\
\text { Sheot } \\
\text { Number }\end{array}$ & $\begin{array}{l}\text { SHllcone OII } \\
\text { Absorption } \\
\text { Duration } \\
\text { (days) }\end{array}$ & \begin{tabular}{|l|} 
Mass \\
Before \\
Test \\
(g)
\end{tabular} & $\begin{array}{l}\text { Extraclion } \\
\text { Solvent }\end{array}$ & \begin{tabular}{|l|} 
Solvent \\
Extracilon \\
Volume \\
(ml) \\
\end{tabular} & \begin{tabular}{|l|} 
Sample \\
Dlluled \\
Volume \\
(ul) \\
\end{tabular} & \begin{tabular}{|l|} 
Solvent \\
Dilulion \\
Volume \\
(ul)
\end{tabular} & \begin{tabular}{|l|} 
Injected \\
Sample \\
Volume \\
(ul)
\end{tabular} & $\begin{array}{l}\text { GC } \\
\text { Peak } \\
\text { Area }\end{array}$ & $\begin{array}{l}\text { Diluted } \\
\text { Sample } \\
\text { Concentratlon } \\
\text { (PPM) }\end{array}$ & $\mid \begin{array}{l}\text { Volume of S1 } \\
\text { Oll In Diluted } \\
\text { Sample } \\
\text { (ml) }\end{array}$ & \begin{tabular}{|l|} 
Aclual \\
Si oll \\
Concentra \\
(PPM)
\end{tabular} & $\begin{array}{l}\text { Actual } \\
\text { Si oll } \\
\text { Volume } \\
\text { (mi) }\end{array}$ & \begin{tabular}{|l|} 
Actual \\
Sl oll \\
Mass \\
(g) \\
\end{tabular} & Solubility \\
\hline 1 & & 1.3014 & hexane & 20 & \begin{tabular}{|r|}
50 \\
\end{tabular} & 1260 & 1.5 & 6635930 & 91.58 & $1.1997 E-04$ & 2399.38 & 0.0480 & 0.0410 & 0.0315 \\
\hline 2 & 2 & & hexane & 20 & 50 & 1260 & 1.5 & & 110.77 & & & & 0.0496 & 0.0381 \\
\hline 3 & 2 & 1.2684 & DCM & 20 & 50 & 1260 & 1.6 & 5878497 & 144.76 & 1.8963E-04 & 3792.69 & 0.0759 & 0.0648 & 0.0511 \\
\hline$\frac{1}{4}$ & $\overline{2}$ & 1.2853 & hexane & 20 & 50 & 1260 & $\overline{1.5}$ & 7958811 & 109.84 & $1.438 \mathrm{BE}-04$ & 2877.70 & 0.0576 & 0.0492 & 0.0382 \\
\hline 5 & $\overline{2}$ & 1.2893 & $\overline{D C M}$ & 20 & 50 & 1260 & 1.6 & 6069275 & 149.46 & $1.9579 \mathrm{E} .04$ & 3915.78 & 0.0783 & 0.0669 & 0.0519 \\
\hline
\end{tabular}

GC Standard Coefficlent Calculation

\begin{tabular}{|l|l|r|l|r|}
\hline Solvent & $\begin{array}{l}\text { Concentratlon } \\
\text { of Prepared } \\
\text { Standard } \\
\text { (PPM) }\end{array}$ & $\begin{array}{l}\text { Standard } \\
\text { Peak } \\
\text { Area }\end{array}$ & $\begin{array}{l}\text { Injected } \\
\text { Standard } \\
\text { Volume } \\
\text { (ul) }\end{array}$ & $\begin{array}{l}\text { Standard } \\
\text { Coefflelent } \\
\text { area/(ul'PPM) }\end{array}$ \\
\hline Hexane & 100 & 6763022 & 1.4 & 48307.3 \\
\hline DCM & 100 & 4060880 & 1.6 & 25380.5 \\
\hline
\end{tabular}

\begin{tabular}{|l|r|}
\hline S.G. & $0 . \overline{854}$ \\
Densiliy of ofl $(\mathrm{g} / \mathrm{cm} 3)$ & 0.8540 \\
\hline
\end{tabular}


Table 7: Absorption Test Raw Data - Gravimetric Test $(.060 \mathrm{~cm})$ thick $(\mathrm{cm})$ 0.060

\begin{tabular}{|c|c|c|c|c|c|}
\hline $\begin{array}{l}\text { Time } \\
\text { (min) }\end{array}$ & $\begin{array}{l}\text { Sample } \\
\text { Mass } \\
\text { (g) }\end{array}$ & \begin{tabular}{|l} 
Absorbed Oil \\
Mass \\
(g) \\
\end{tabular} & $\begin{array}{l}\text { Solubility } \\
(\mathrm{g} / \mathrm{g}) \\
\end{array}$ & \begin{tabular}{|l} 
Root \\
Time \\
$(\min )^{\wedge} .5$ \\
\end{tabular} & $\begin{array}{l}\text { Fractional } \\
\text { Absorbance } \\
(\mathrm{g} / \mathrm{g})\end{array}$ \\
\hline$\overline{0}$ & 1.0926 & 0.0000 & 0.0000 & 0.00 & 0.000 \\
\hline 5 & 1.0987 & 0.0061 & 0.0056 & 2.24 & 0.145 \\
\hline 10 & 1.1007 & 0.0081 & 0.0074 & 3.16 & 0.193 \\
\hline 15 & 1.1025 & 0.0099 & 0.0091 & 3.87 & 0.236 \\
\hline 20 & 1.1041 & 0.0115 & 0.0105 & 4.47 & 0.274 \\
\hline 30 & 1.1076 & 0.0150 & 0.0137 & 5.48 & 0.357 \\
\hline 40 & 1.1087 & 0.0161 & 0.0147 & 6.32 & 0.383 \\
\hline 50 & 1.1095 & 0.0169 & 0.0155 & 7.07 & 0.402 \\
\hline 65 & 1.1111 & 0.0185 & 0.0169 & 8.06 & 0.440 \\
\hline 80 & 1.1128 & 0.0202 & 0.0185 & 8.94 & 0.481 \\
\hline 100 & 1.1149 & 0.0223 & 0.0204 & 10.00 & 0.531 \\
\hline 120 & 1.1171 & 245 & 0.0224 & 10.95 & 0.583 \\
\hline 140 & 1.1186 & 260 & 0.0238 & 11.83 & 0.619 \\
\hline 160 & 1.1201 & 0.0275 & 0.0252 & 12.65 & 0.655 \\
\hline 190 & 1.1220 & 294 & 0.0269 & 13.78 & 0.700 \\
\hline 220 & 1.1236 & & 0.0284 & 14.83 & 0.738 \\
\hline 250 & 1.1250 & & 0.0297 & 15.81 & 0.771 \\
\hline 280 & 1.1265 & & 0.0310 & 16.73 & 0.807 \\
\hline 325 & 1.1278 & 352 & 0.0322 & 18.03 & 0.838 \\
\hline 355 & 1.1282 & 356 & 0.0326 & 18.84 & 0.848 \\
\hline 415 & 1.1293 & & 0.0336 & 20.37 & 0.874 \\
\hline 475 & 1.1307 & & 0.0349 & 21.79 & 0.907 \\
\hline 535 & 1.1319 & & 0.0360 & 23.13 & 0.936 \\
\hline 595 & 1.1321 & & 362 & 24.39 & 0.940 \\
\hline 655 & 1.1329 & & 0.0369 & 25.59 & 0.960 \\
\hline 1435 & 1.1346 & 220 & 0.0384 & 37.88 & 1.000 \\
\hline 1555 & 1.1344 & 0.0418 & 0.0383 & 39.43 & 0.995 \\
\hline 1855 & 1.1344 & 0.0418 & 0.0383 & 43.07 & 0.995 \\
\hline
\end{tabular}


Table 8: Absorption Test Raw Data - Gravimetric Test $(.065 \mathrm{~cm})$ thick(cm) 0.065

\begin{tabular}{|c|c|c|c|c|c|}
\hline $\begin{array}{l}\text { Time } \\
\text { (min) }\end{array}$ & $\begin{array}{l}\text { Sample } \\
\text { Mass } \\
\text { (g) } \\
\end{array}$ & \begin{tabular}{|l} 
Absorbed Oil \\
Mass \\
(g) \\
\end{tabular} & $\begin{array}{l}\text { Solubility } \\
(g / g) \\
\end{array}$ & $\begin{array}{l}\text { Root } \\
\text { Time } \\
(\text { min })^{\wedge} .5\end{array}$ & $\begin{array}{l}\text { Fractional } \\
\text { Absorbance } \\
(g / g)\end{array}$ \\
\hline 0 & 1.2306 & 0.0000 & 0.0000 & 0.00 & 0.000 \\
\hline 5 & 1.2345 & 0.0039 & 0.0032 & 2.24 & 0.060 \\
\hline 10 & 1.2379 & 0.0073 & 0.0059 & 3.16 & 0.113 \\
\hline 15 & 1.2408 & 0.0102 & 0.0083 & 3.87 & 0.157 \\
\hline 20 & 1.2431 & 0.0125 & 0.0102 & 4.47 & 0.193 \\
\hline 25 & 1.2450 & 0.0144 & 0.0117 & 5.00 & 0.222 \\
\hline 30 & 1.2461 & 0.0155 & 0.0126 & 5.48 & 0.239 \\
\hline 35 & 1.2477 & 0.0171 & 0.0139 & 5.92 & 0.264 \\
\hline$\overline{40}$ & 1.2486 & 0.0180 & 0.0146 & 6.32 & 0.278 \\
\hline 45 & 1.2499 & 0.0193 & 0.0157 & 6.71 & 0.298 \\
\hline 50 & 1.2512 & 0.0206 & 0.0167 & 7.07 & 0.318 \\
\hline 55 & 1.2529 & 0.0223 & 0.0181 & 7.42 & 0.344 \\
\hline 60 & 1.2538 & 0.0232 & 0.0189 & 7.75 & 0.358 \\
\hline 65 & 1.2547 & 0.0241 & 0.0196 & 8.06 & 0.372 \\
\hline 75 & 1.2567 & 0.0261 & 0.0212 & 8.66 & 0.403 \\
\hline 85 & 1.2582 & 0.0276 & 0.0224 & 9.22 & 0.426 \\
\hline 100 & 1.2604 & 0.0298 & 0.0242 & 10.00 & 0.460 \\
\hline 120 & 1.2640 & 0.0334 & 0.0271 & 10.95 & 0.515 \\
\hline 150 & 1.2682 & 0.0376 & 0.0306 & 12.25 & 0.580 \\
\hline 180 & 1.2713 & & 0.0 & $\overline{13.42}$ & 0.628 \\
\hline 210 & 1.2744 & & 0.0 & 14.49 & 0.676 \\
\hline 240 & 1.2765 & & 0.0373 & 15.49 & 0.708 \\
\hline 1060 & 1.2952 & & 0.0525 & 32.56 & 0.997 \\
\hline 1120 & 1.2954 & 0.0 & 0.0527 & 33.47 & 1.000 \\
\hline 9180 & 1.2950 & 0.0644 & 0.0523 & 34.35 & 0.994 \\
\hline
\end{tabular}


Table 9: Absorption Test Data Results - Gravime

\begin{tabular}{|c|c|c|c|}
\hline $\begin{array}{l}\text { Test } \\
\text { Sample }\end{array}$ & $\begin{array}{l}\text { Diffusion } \\
\text { Coefficient } \\
D \\
(\mathrm{~cm} / \mathrm{s})\end{array}$ & $\begin{array}{l}\text { Solubility } \\
\text { Coefficient } \\
\mathrm{S} \\
(\mathrm{g} / \mathrm{g}) \\
\end{array}$ & $\begin{array}{l}\text { Permeability } \\
\text { Coefficient } \\
\text { P } \\
(\mathrm{cm} 2 / \mathrm{s})\end{array}$ \\
\hline B_.060 & 2.86E-08 & 0.0384 & 1.1E-09 \\
\hline B.065 & 3.62E-08 & 0.0526 & $2.35 E-09$ \\
\hline
\end{tabular}



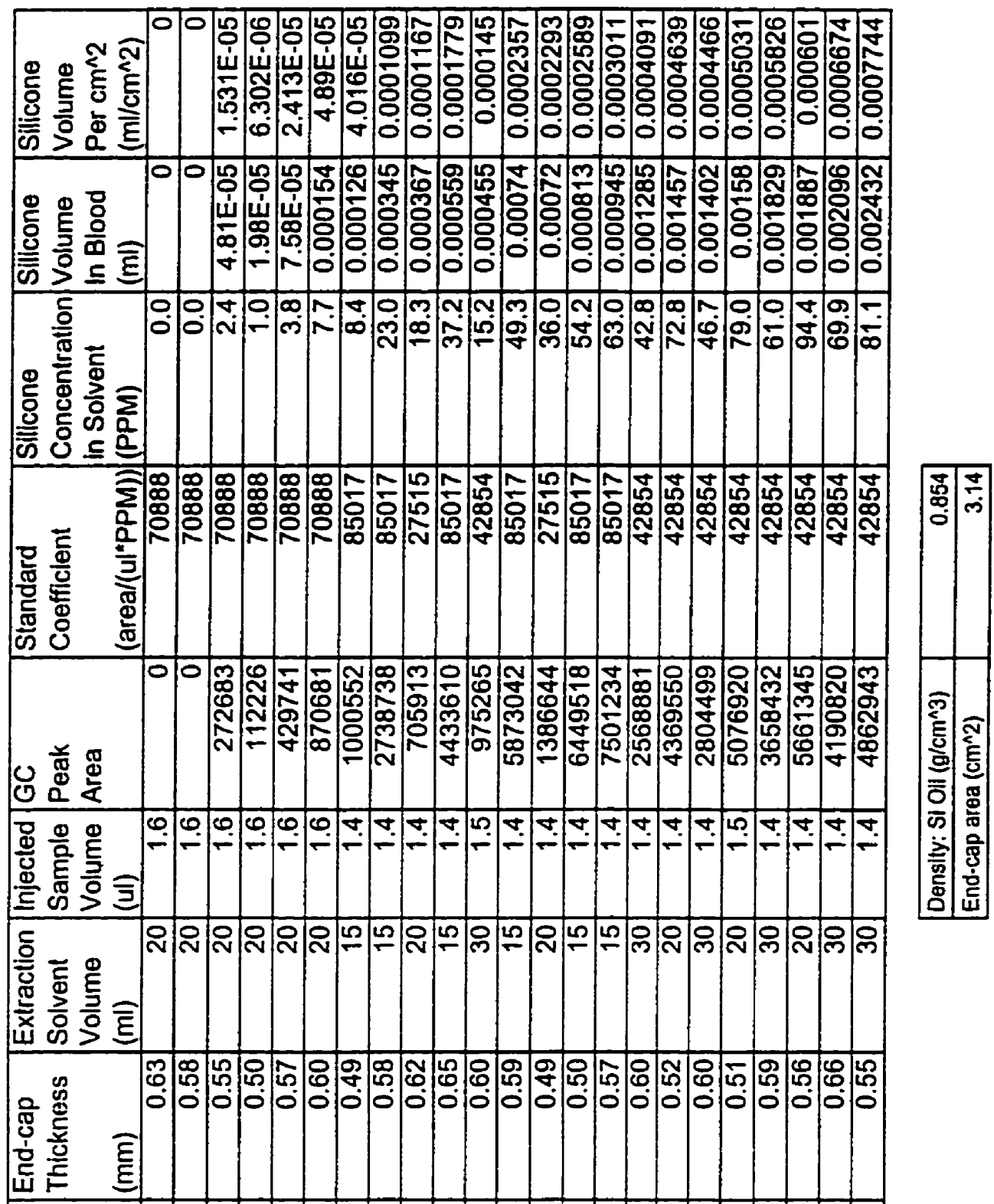

爻

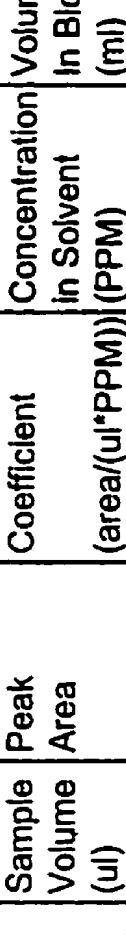

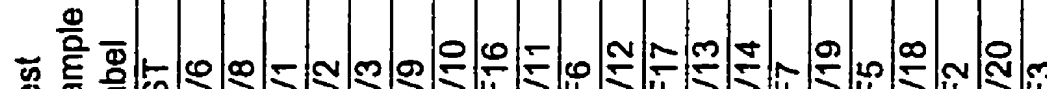

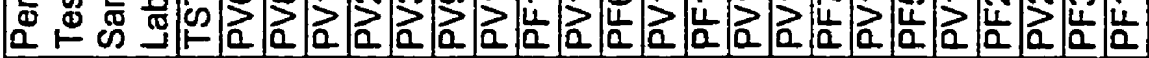

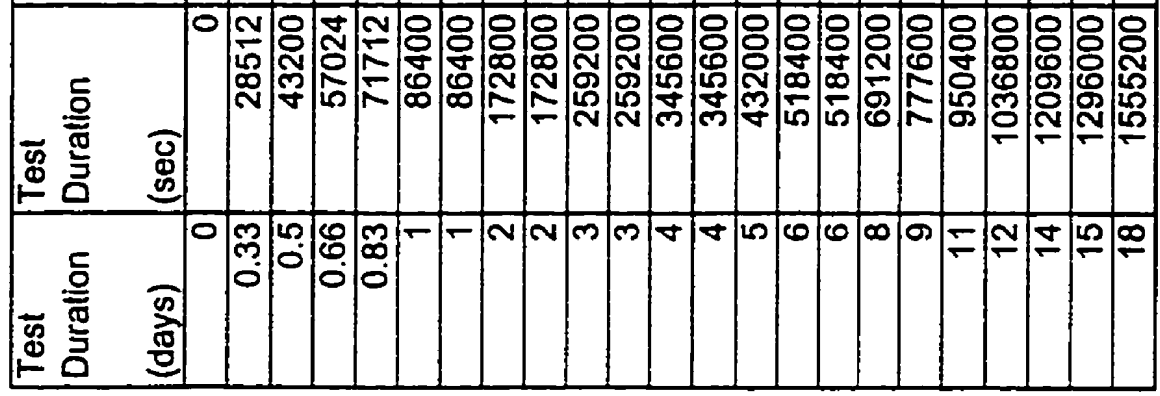


Table 11: Permeability Test Data Results

\begin{tabular}{|c|c|c|c|}
\hline $\begin{array}{l}\text { Average } \\
\text { Sample } \\
\text { Thickness } \\
\text { (cm) }\end{array}$ & $\begin{array}{l}\text { Diffusion } \\
\text { Coefficient } \\
D \\
\text { (cm2/s) }\end{array}$ & $\begin{array}{l}\text { Solubility } \\
\text { Coefficient } \\
\text { s } \\
(g / g)\end{array}$ & $\begin{array}{l}\text { Permeability } \\
\text { Coefficient } \\
P \\
(\mathrm{~cm} 2 / \mathrm{s})\end{array}$ \\
\hline 0.0596 & 3.07E-08 & 0.001332 & 4.1E-11 \\
\hline
\end{tabular}

Table 12: Predicted Silicone Oil Loss Through Blood Chamber Diaphragm Used permeability coefficient from the permeability tests.

\begin{tabular}{|l|r|r|}
\hline Time & Time & $\begin{array}{l}\text { Volume Permeating } \\
\text { Through 60.5 cm2 } \\
\text { Diaphragm } \\
(\mathrm{ml})\end{array}$ \\
\hline 1 week & 604800 & 0.025 \\
\hline 1 month & 2629744 & 0.109 \\
\hline 1 year & 31600000 & 1.312 \\
\hline 3 years & 94700000 & 3.937 \\
\hline 9 years & $2.84 \mathrm{E}+08$ & 11.811 \\
\hline
\end{tabular}




\section{APPENDIX D:}

Gas Chromatography and Mass Spectrum Data 
The gas chromatography testing was performed on a Varian Saturn II GC with a $\mathbf{2 3 0 0}$ mass spectrometer. A DB5 column was used with a length of $25 \mathrm{~m}$, outer diameter of $.25 \mathrm{~mm}$ and .25 micron film thickness. A septum programable injector was used and helium was the carrier gas.

Table 13: GC Method

\begin{tabular}{|r|r|r|r|r|}
\hline Segment & $\begin{array}{l}\text { Temperature } \\
\text { (C) }\end{array}$ & $\begin{array}{l}\text { Rate } \\
\text { (deg/min) }\end{array}$ & $\begin{array}{l}\text { Time } \\
\text { (min) }\end{array}$ & $\begin{array}{l}\text { Total Time } \\
\text { (min) }\end{array}$ \\
\hline start & 50 & & & 0.00 \\
\hline 1 & 50 & 0.0 & 2.00 & 2.00 \\
\hline 2 & 155 & 15.0 & 7.00 & 9.00 \\
\hline 3 & 300 & 25.0 & 5.80 & 14.80 \\
\hline 4 & 300 & 0.0 & 5.20 & 20.00 \\
\hline
\end{tabular}

\begin{tabular}{|l|l|l|l|l|}
\cline { 2 - 5 } \multicolumn{2}{l|}{} & $\begin{array}{l}\text { Column } \\
\text { Temperature } \\
\text { (C) }\end{array}$ & $\begin{array}{l}\text { Injector } \\
\text { Temperature } \\
\text { (C) }\end{array}$ & $\begin{array}{l}\text { Xfer Line } \\
\text { Temperature } \\
\text { (C) }\end{array}$ \\
\hline Valve 1 & set & 50 & 280 & 250 \\
\hline Valve 2 & actual & 300 & 280 & 250 \\
\hline
\end{tabular}

Table 14: ACU Method

\begin{tabular}{|l|l|}
\hline Parameter & Value \\
\hline Mass Range & 50 to 500 amu \\
\hline Seconds/Scan & 1.000 (3u Scans) \\
\hline Acquire Time & $20 \mathrm{~min}$ \\
\hline Fil/Mul Delay & $160 \mathrm{sec}$ \\
\hline Peak Threshold & 3 counts \\
\hline Mass Defect & $0 \mathrm{mmu} / 100$ amu \\
\hline Background Mass & 50 amo \\
\hline lonize Mode & El \\
\hline Auto lon Conirol & On \\
\hline Cal. Gas & Off \\
\hline
\end{tabular}


Figure 46: Gas Chromatography Sample Graph - Standard

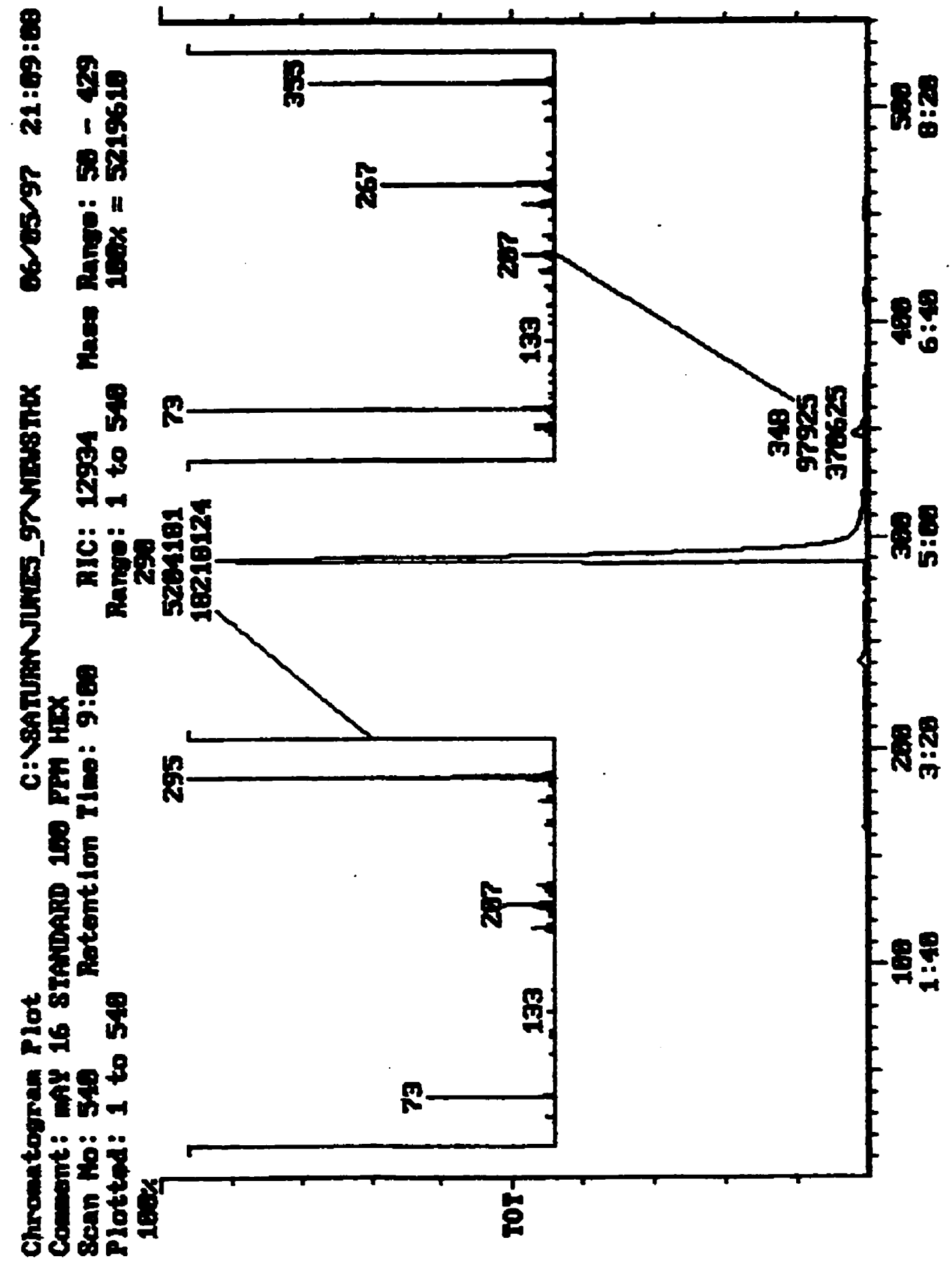


Figure 47: Gas Chromatography Mass Spectrum Sample Graph - Standard

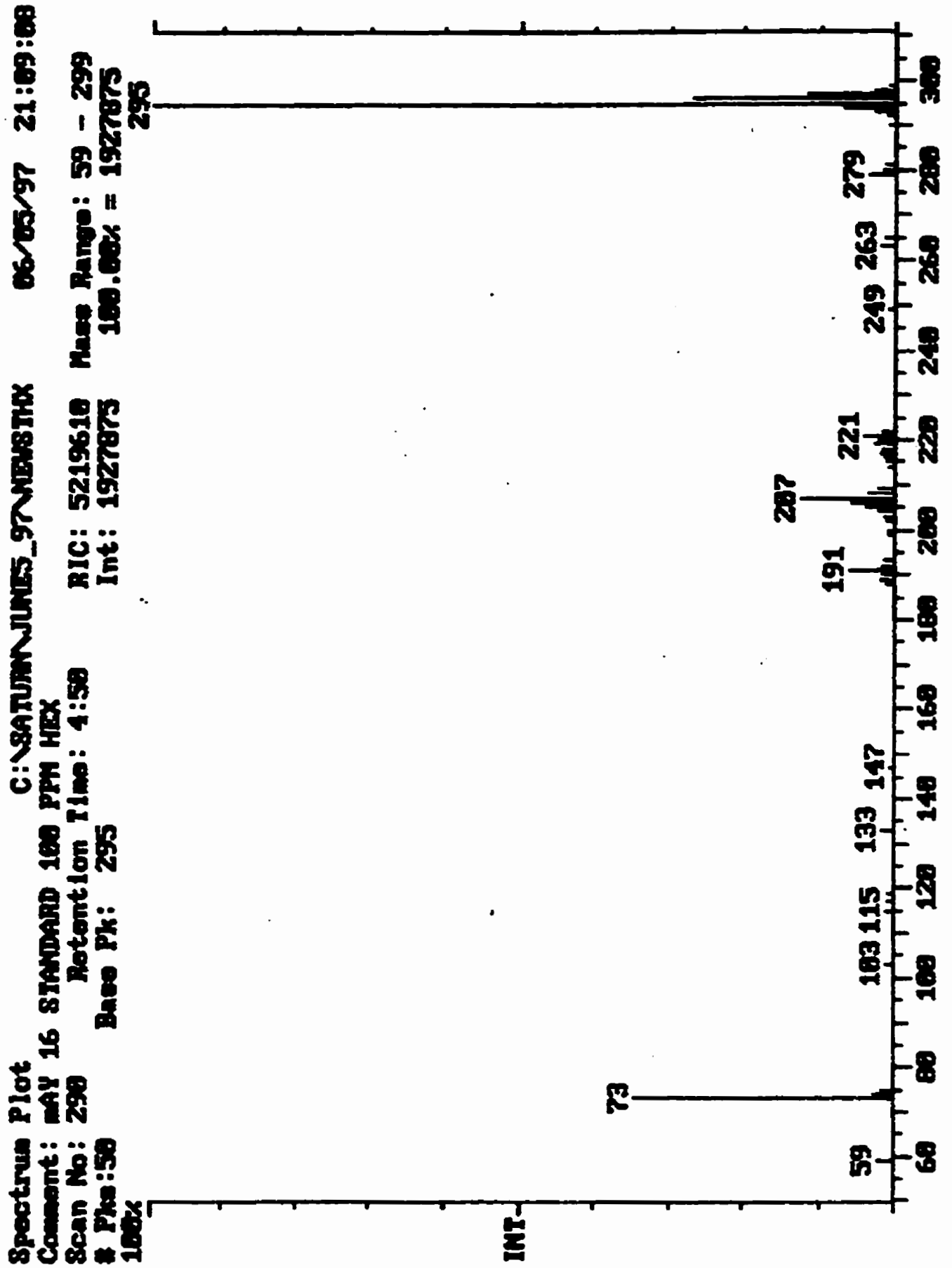


Table 15: Gas Chromatography Sample Mass List - Standard

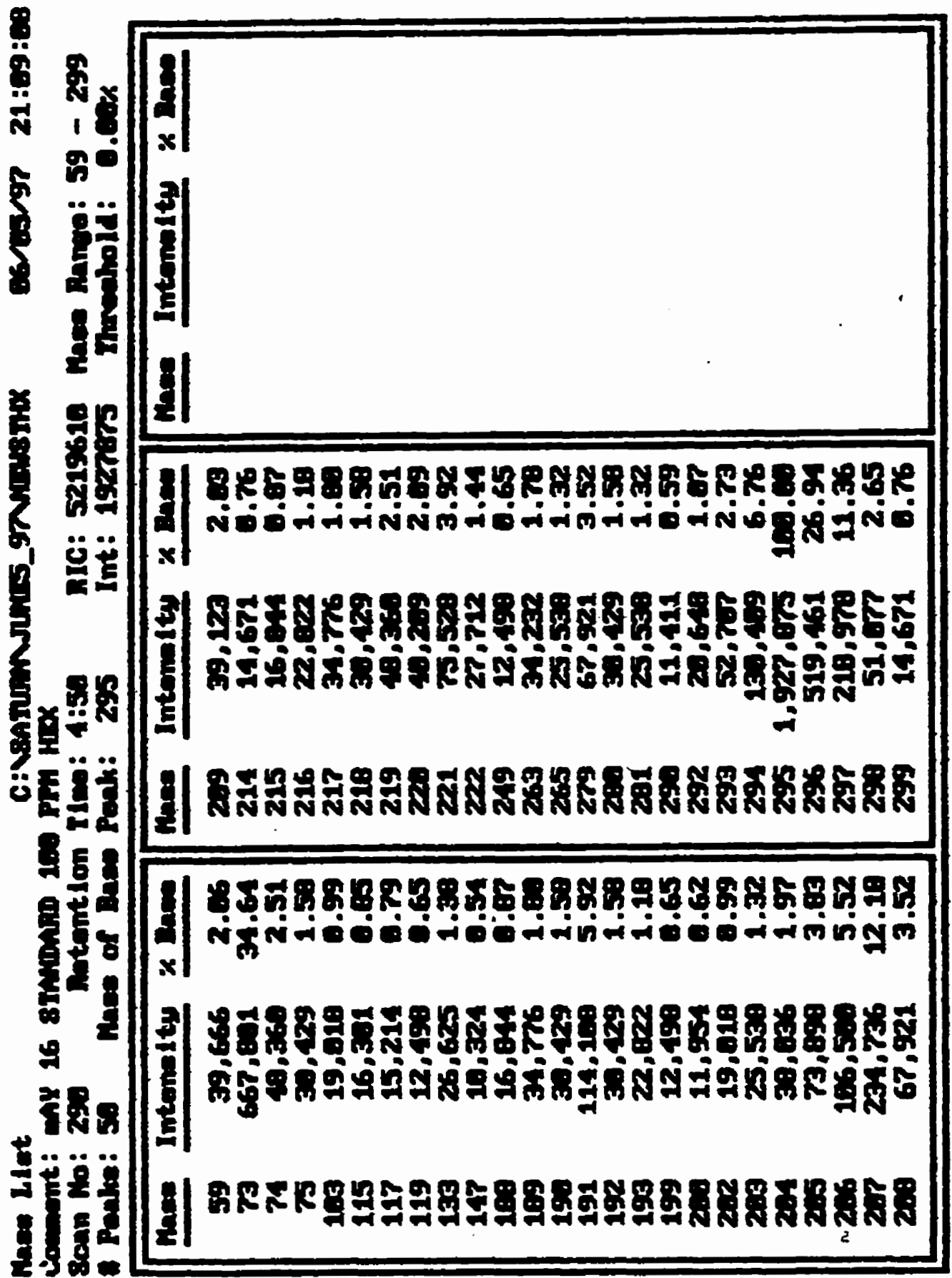


Figure 48: Gas Chromatography Sample Graph - Sample PV12

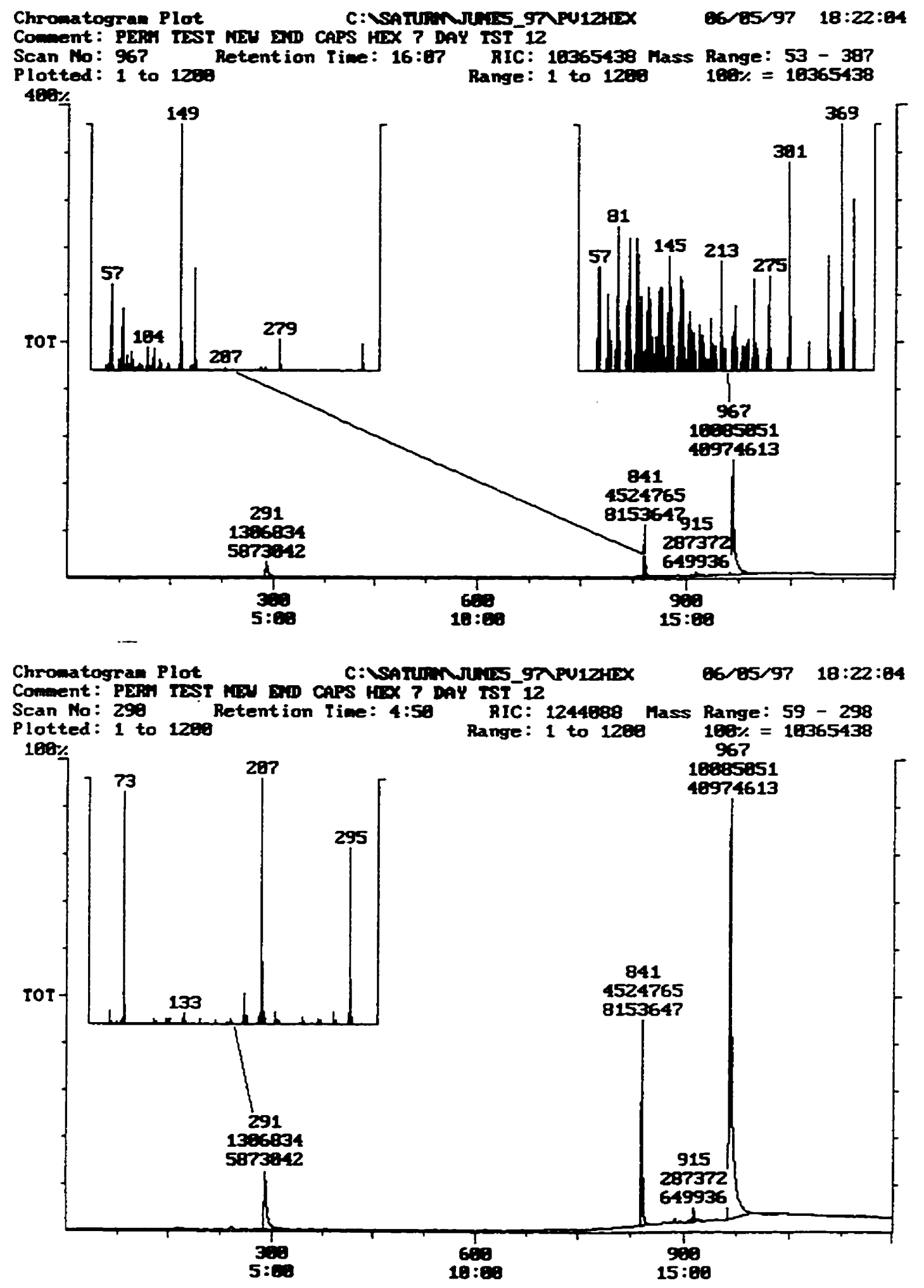


Figure 49: Gas Chromatography Mass Spectrum Sample Graph

- Sample PV12

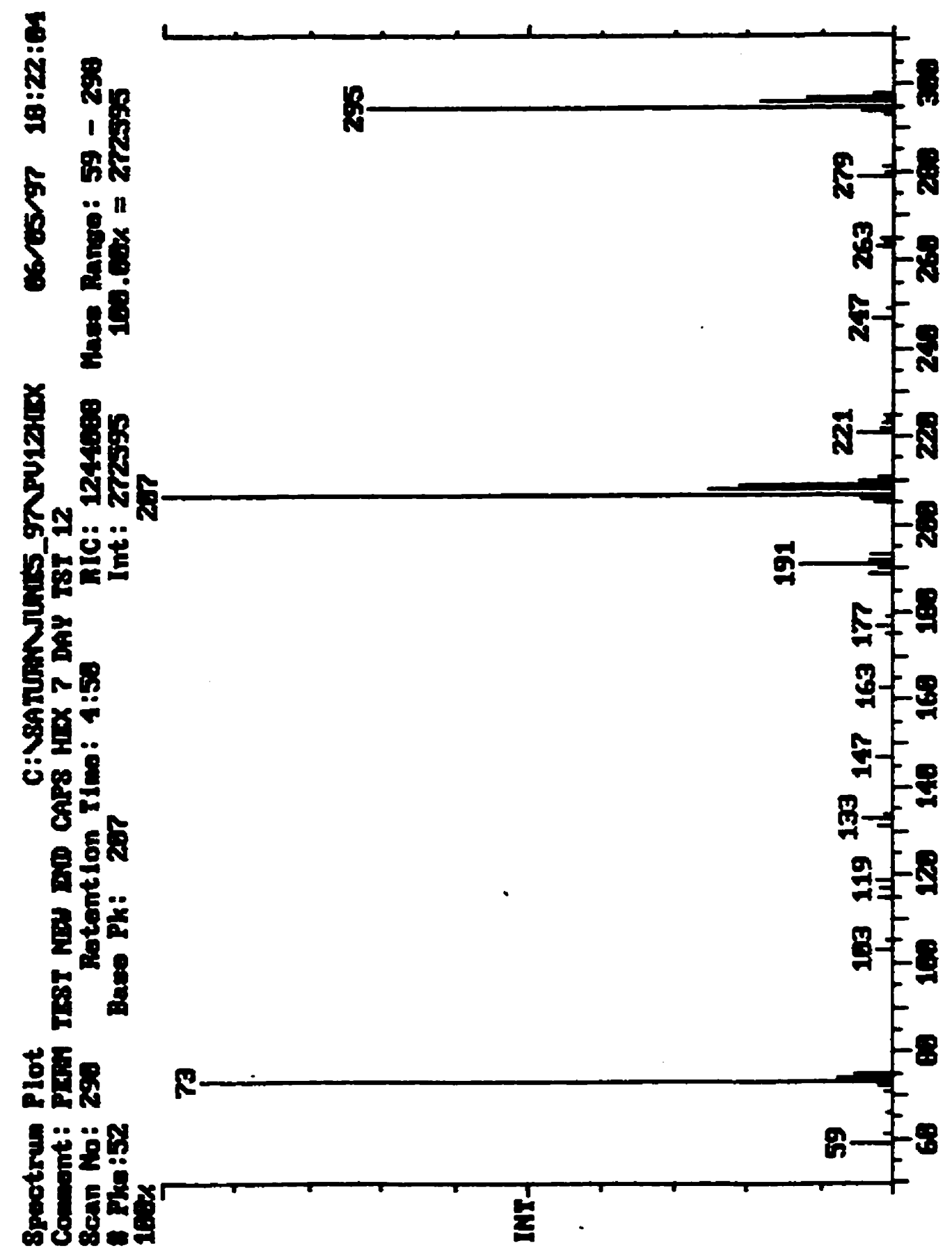


Table 16: Gas Chromatography Sample Mass List - Sample PV12

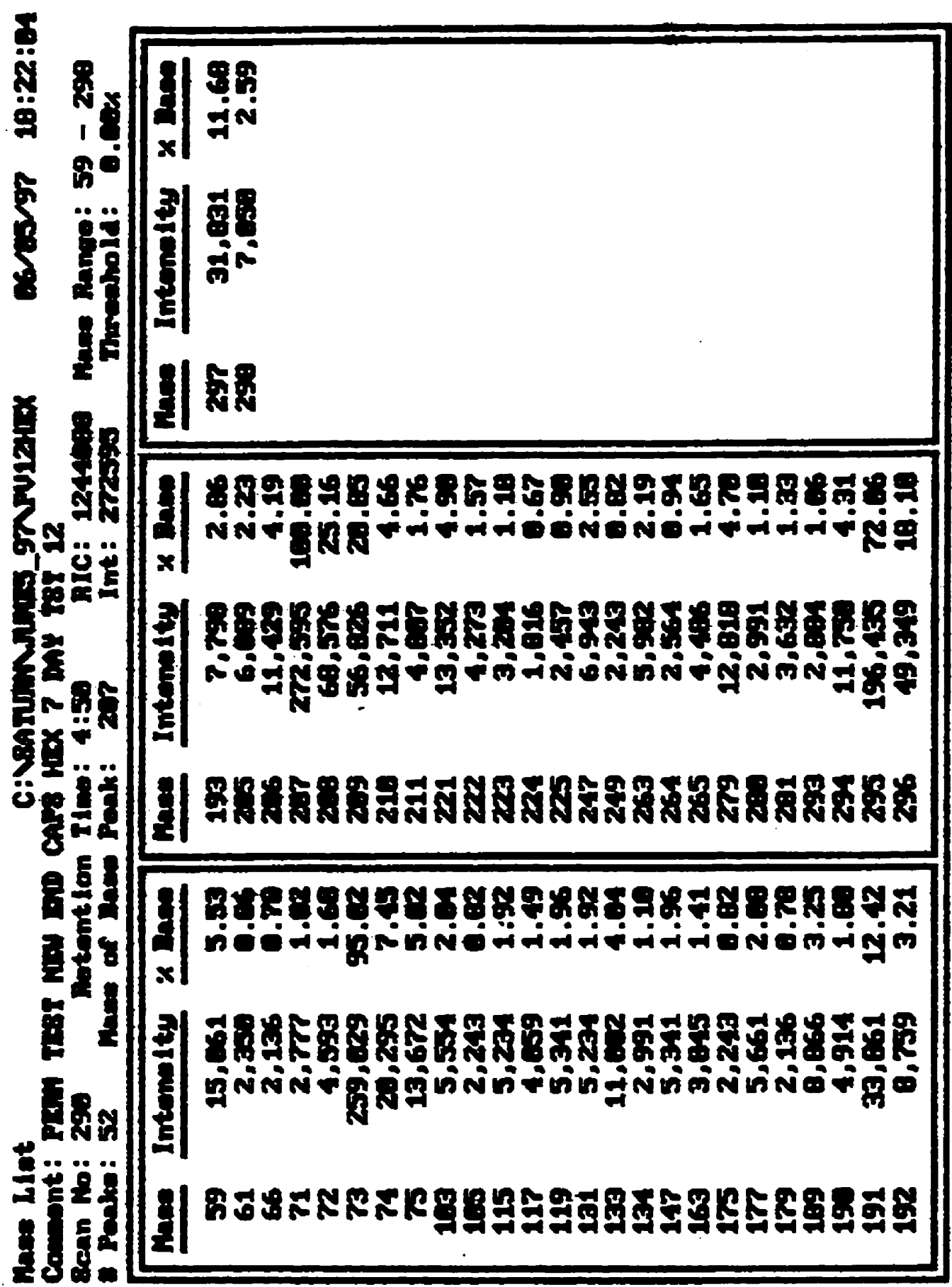



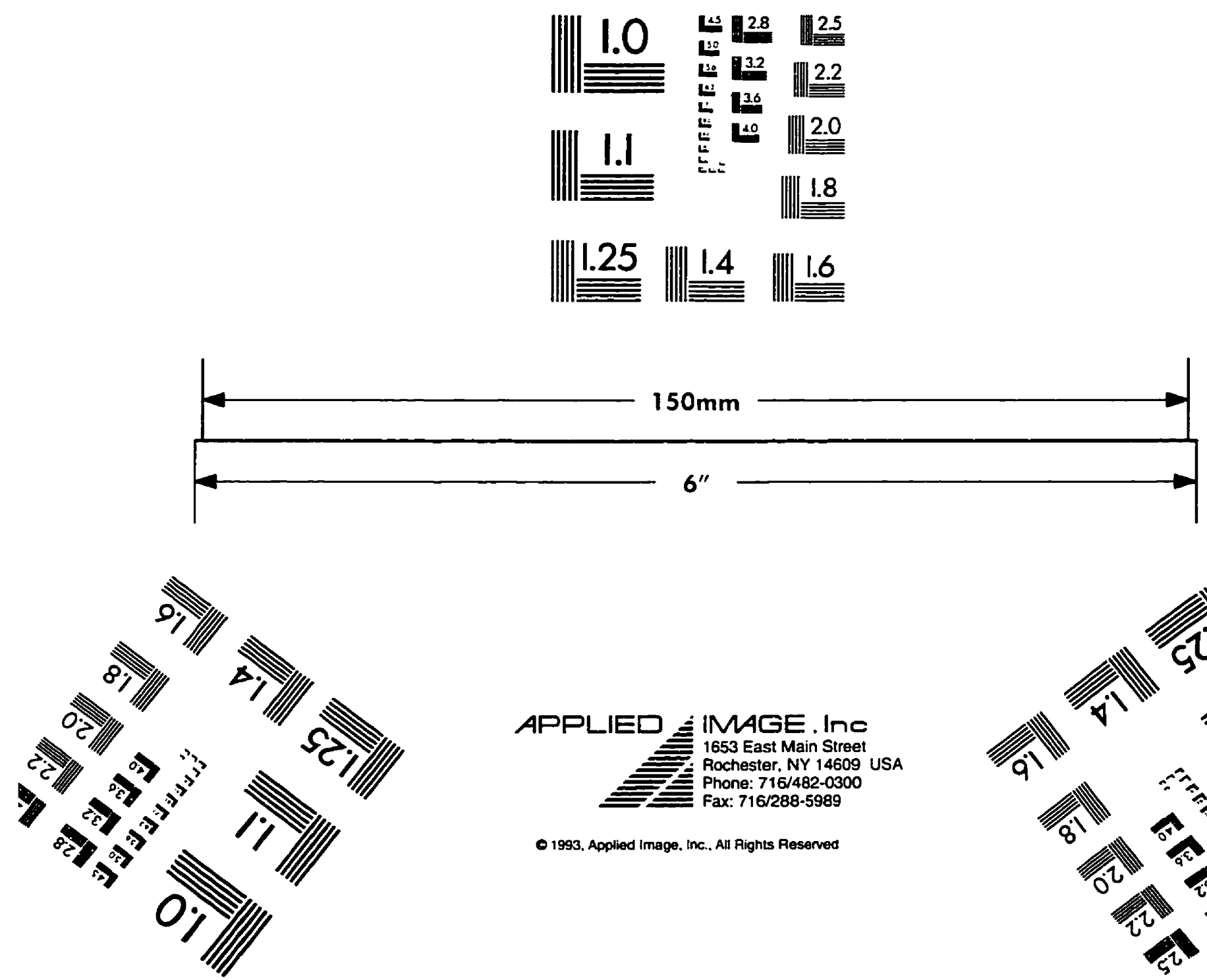

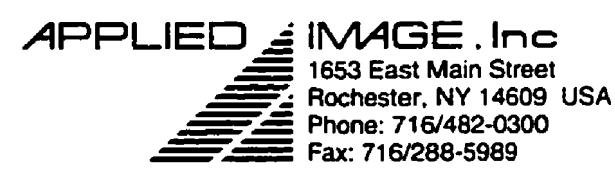

- 1993, Applied Image. Inc.. All Aights Reserved

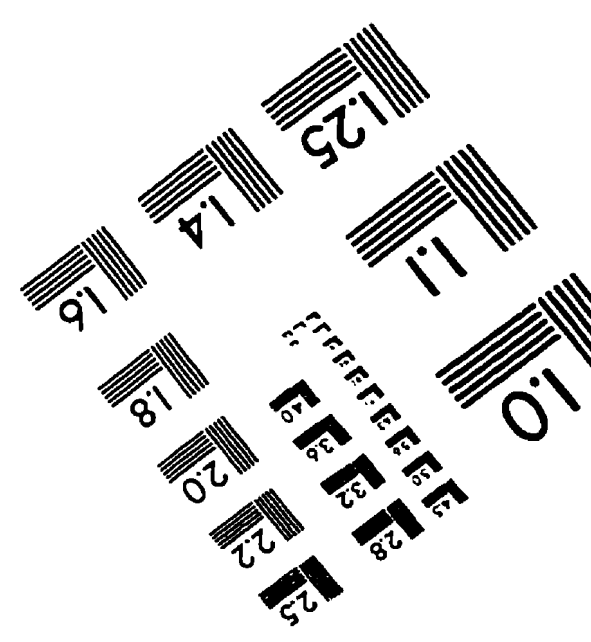

